\title{
Decision-Making Analysis Based on Fuzzy Graph Structures
}

\author{
Ali N. A. Koam (iD), ${ }^{1}$ Muhammad Akram ${ }^{(D}{ }^{2}$ and Peide Liu ${ }^{3}{ }^{3}$ \\ ${ }^{1}$ Department of Mathematics, College of Science, Jazan University, New Campus, P.O. Box 2097, Jazan 45142, Saudi Arabia \\ ${ }^{2}$ Department of Mathematics, University of the Punjab, New Campus, Lahore, Pakistan \\ ${ }^{3}$ School of Management Science and Engineering, Shandong University of Finance and Economics, Jinan 250014, \\ Shandong, China \\ Correspondence should be addressed to Muhammad Akram; m.akram@pucit.edu.pk
}

Received 13 June 2020; Accepted 8 July 2020; Published 12 August 2020

Guest Editor: S. A. Edalatpanah

Copyright $\odot 2020$ Ali N. A. Koam et al. This is an open access article distributed under the Creative Commons Attribution License, which permits unrestricted use, distribution, and reproduction in any medium, provided the original work is properly cited.

\begin{abstract}
A graph structure is a useful framework to solve the combinatorial problems in various fields of computational intelligence systems and computer science. In this research article, the concept of fuzzy sets is applied to the graph structure to define certain notions of fuzzy graph structures. Fuzzy graph structures can be very useful in the study of various structures, including fuzzy graphs, signed graphs, and the graphs having labeled or colored edges. The notions of the fuzzy graph structure, lexicographic-max product, and degree and total degree of a vertex in the lexicographic-max product are introduced. Further, the proposed concepts are explained through several numerical examples. In particular, applications of the fuzzy graph structures in decision-making process, regarding detection of marine crimes and detection of the road crimes, are presented. Finally, the general procedure of these applications is described by an algorithm.
\end{abstract}

\section{Introduction}

Decision-making is a process of solving problems for choosing the best alternative. Multicriteria decision-making (MCDM) can be explicated as a discipline of operation research in which the satisfactory solution is opted regarding some significant factors which can be considered as conflicting criteria for the decision-making problems. The concept of MCDM is widely used in medical, economics, engineering, and social sciences. The process of MCDM can be classified according to the number of decision makers (DMs), i.e., individual decisionmaking and group decision-making (GDM). Multicriteria group decision-making (MCGDM) is a procedure to deal with the problems to find the most suitable alternative relative to some criteria by a cooperative group of decision makers. In MCGDM, the individual decision matrices of all DMs are merged to get a group satisfactory solution.

Most of the classical frameworks of formal modeling and reasoning are crisp and deterministic in the character. Crisp is yes or no type and does not deal more or less type. In the traditional dual logic, a statement is either true or false and nothing is in between. In the set theory, an element either belongs to the set or not. A fuzzy set, proposed by Zadeh [1], is the class of objects with continuity of grades of membership. It is characterized by the membership function that assigns each element grade of membership between zero and one. Nowadays, in modeling uncertain systems of the industry, society, and nature, the theory of fuzzy sets plays a vital role. In the decision making-process, it is used as a strong mathematical tool facilitating the approximate reasoning.

A more convenient way to illustrate data and information regarding interactions between elements or objects is a graph $[2,3]$. Due to uncertainty in representation of elements or in their relations, it is quite natural to develop a fuzzy graph. A fuzzy graph is defined as a symmetric and binary fuzzy relation on the fuzzy subset [4]. Rosenfeld [5] considered fuzzy graphs based on fuzzy relations [6]. Later on, Bhattacharya [7] gave remarks on fuzzy graphs. Sunitha and Vijayakumar [8] worked on the complement of fuzzy graphs. Mordeson and Nair [9] discussed various properties of fuzzy graphs and fuzzy hypergraphs. Nagoor Gani and Radha $[10,11]$ introduced the notions of regular fuzzy graphs, totally regular fuzzy graphs, and degree and total degree of a vertex in some fuzzy graphs. Bhutani and Battou [12] studied novel concepts of M-strong fuzzy graphs. Matrix representation of graphs under fuzzy information was proposed by Chen [13]. For further studies on fuzzy graphs, the readers are referred to [14-34]. 
Sampathkumar [35] introduced the concept of graph structures. Graph structures prove to be very useful in the study of various areas of computational intelligence and computer science. The lexicographic product was first considered by Hausdorff [36]. The lexicographic product of two fuzzy graphs was introduced by Radha and Arumugam [37]. Dinesh [38] worked on fuzzy graph structures and discussed few related concepts. Ramakrishnan and Dinesh [39-41] discussed generalized fuzzy graph structures. Akram and Sitara $[42,43]$ introduced the semistrong min-product and maximal product of fuzzy graph structures and investigated their corresponding properties. Residue product of fuzzy graph structures was studied by Akram et al. [44]. In this research article, the concept of fuzzy sets is applied to a graph structure to define certain notions of fuzzy graph structures. The notions of the fuzzy graph structure, lexicographic-max product, and degree and total degree of a vertex in lexicographic-max product are introduced. Further, the proposed concepts are explained through several numerical examples. In particular, applications of the fuzzy graph structures in decision-making process, regarding detection of marine crimes and detection of the road crimes, are presented. Finally, the general procedure of these applications is described by an algorithm.

The contents of this article are as follows: In Section 2, some fundamental concepts of graph structures and fuzzy graphs are reviewed. The certain notions of fuzzy graph structures are defined, and their interesting properties are investigated in the same section. Section 3 illustrates the applicability of fuzzy graph structures in real-life phenomena. The whole article is concluded in Section 4.

\section{Certain Notions of Fuzzy Graph Structures}

Definition 1 (see [35]). A graph structure (GS) $G^{*}=$ $\left(V, R_{1}, \ldots, R_{n}\right)$ consists of a nonempty set $V$ together with relations $R_{1}, R_{2}, \ldots, R_{n}$ on $V$ which are mutually disjoint such that each $R_{i}, 1 \leq i \leq n$, is symmetric and irreflexive. One can represent a graph structure $G^{*}=\left(V, R_{1}, \ldots, R_{n}\right)$ in the plane just like a graph where each edge is labeled as $R_{i}$, $1 \leq i \leq n$.

Definition 2 (see [38]). Let $\alpha$ be the fuzzy set on set $V$ and $v_{1}, v_{2}, \ldots, v_{n}$ be fuzzy sets on $R_{1}, R_{2}, \ldots, R_{n}$, respectively. If $0 \leq v_{i}(v u) \leq \alpha(v) \wedge \alpha(u) \forall u, v \in V, \quad i=1,2, \ldots, n, \quad$ then $\widehat{G}=\left(\alpha, v_{1}, v_{2}, \ldots, v_{n}\right)$ is called the fuzzy graph structure (FGS) of the graph structure $G^{*}$. If $v u \in \sup p\left(v_{i}\right)$, then $v u$ is named as the $v_{i}$-edge of FGS $\widehat{G}$.

Definition 3. Let $\widehat{G}_{1}=\left(\alpha_{1}, v_{1}^{\prime}, v_{2}^{\prime}, \ldots, v_{n}^{\prime}\right)$ and $\widehat{G}_{2}=\left(\alpha_{2}\right.$, $\left.v_{1}^{\prime \prime}, v_{2}^{\prime \prime}, \ldots, v_{n}^{\prime \prime}\right)$ be two fuzzy graph structures (FGSs) having underlying crisp graph structures (GSs) $\widehat{G}_{1}^{*}=\left(V_{1}, R_{1}^{\prime}\right.$, $\left.R_{2}^{\prime}, \ldots, R_{n}^{\prime}\right)$ and $\widehat{G}_{2}^{*}=\left(V_{2}, R_{1}^{\prime \prime}, R_{2}^{\prime \prime}, \ldots, R_{n}^{\prime \prime}\right)$, respectively. A fuzzy graph structure $\widehat{G}=\left(\alpha, v_{1}, v_{2}, \ldots v_{n}\right)$ with an underlying crisp graph structure $\widehat{G}^{*}=\left(V, R_{1}, R_{2}, \ldots, R_{n}\right)$, where $V=V_{1} \times V_{2}$ and $R_{i}=\left\{\left(v_{1}, w_{1}\right)\left(v_{2}, w_{2}\right) / v_{1}=v_{2}, w_{1} w_{2} \in R_{i}^{\prime \prime}\right.$ or $\left.v_{1} v_{2} \in R_{i}^{\prime}\right\}$, is defined.

The fuzzy vertex set $\alpha$ is defined as $\alpha(v, w)=\alpha_{1}(v) \vee$ $\alpha_{2}(w)$, for all $(v, w) \in V=V_{1} \times V_{2}$.

Fuzzy relations $v_{i}$ are defined as

$$
v_{i}\left(\left(v_{1}, w_{1}\right)\left(v_{2}, w_{2}\right)\right)= \begin{cases}v_{i}^{\prime}\left(v_{1} v_{2}\right), & v_{1} v_{2} \in R_{i}^{\prime}, \\ \alpha_{1}\left(v_{1}\right) \vee v_{i}^{\prime \prime}\left(w_{1} w_{2}\right), & v_{1}=v_{2}, w_{1} w_{2} \in R_{i}^{\prime \prime}, \\ i=1,2, \ldots, n\end{cases}
$$

$\widehat{G}=\left(\alpha, v_{1}, v_{2}, \ldots v_{n}\right)$ is called the lexicographic-max product of $\widehat{G}_{1}$ and $\widehat{G}_{2}$ and is denoted by $\widehat{G}_{1}\left[\widehat{G}_{2}\right]_{\max }$.

Example 1. Consider two FGSs $\widehat{G}_{1}=\left(\alpha_{1}, v_{1}^{\prime}, v_{2}^{\prime}\right)$ and $\widehat{G}_{2}=$ $\left(\alpha_{2}, v_{1}^{\prime \prime}, v_{2}^{\prime \prime}\right)$ with underlying crisp GSs $\widehat{G}_{1}^{*}=\left(V_{1}, R_{1}^{\prime}, R_{2}^{\prime}\right)$ and $\widehat{G}_{2}^{*^{\prime}}=\left(V_{2}, R_{1}^{\prime \prime}, R_{2}^{\prime \prime}\right)$, respectively, which are shown in Figure 1 , where $R_{1}^{\prime}=\left\{v_{1} v_{2}\right\}, R_{2}^{\prime}=\left\{v_{3} v_{4}\right\}, R_{1}^{\prime \prime}=\left\{w_{1} w_{2}\right\}$, and $R_{2}^{\prime \prime}=\left\{w_{2} w_{3}\right\}$.

The lexicographic-max product of the above FGSs $\widehat{G}_{1}=$ $\left(\alpha_{1}, v_{1}^{\prime}, v_{2}^{\prime}\right)$ and $\widehat{G}_{2}=\left(\alpha_{2}, v_{1}^{\prime \prime}, v_{2}^{\prime \prime}\right)$ is shown in Figure 2.

In the lexicographic-max product, $v_{i}^{\prime}$ and $v_{i}^{\prime \prime}$ edges belong to the $v_{i}$ fuzzy set.

Theorem 1. If $\widehat{G}_{1}=\left(\alpha_{1}, v_{1}^{\prime}, v_{2}^{\prime}, \ldots, v_{n}^{\prime}\right)$ and $\widehat{G}_{2}=\left(\alpha_{2}, v_{1}^{\prime \prime}\right.$, $\left.v_{2}^{\prime \prime}, \ldots, v_{n}^{\prime \prime}\right)$ are two effective FGSs such that $\alpha_{2} \geq \alpha_{1}$ and $\nu_{i}^{\prime}$ and $v_{i}^{\prime \prime}$ are constant functions having similar values, then the lexicographic-max product of $\widehat{G}_{1}$ and $\widehat{G}_{2}$ is an effective FGS.
Proof. Let $\widehat{G}_{1}=\left(\alpha_{1}, v_{1}^{\prime}, v_{2}^{\prime}, \ldots, v_{n}^{\prime}\right)$ and $\widehat{G}_{2}=\left(\alpha_{2}, v_{1}^{\prime \prime}, v_{2}^{\prime \prime}, \ldots\right.$, $\left.v_{n}^{\prime \prime}\right)$ be two effective FGSs such that $\alpha_{2} \geq \alpha_{1}$ and $\nu_{i}^{\prime}$ and $\nu_{i}^{\prime \prime}$ be constant functions having similar values, then the definition of the lexicographic-max product provides

Case 1: $v_{1} v_{2} \in R_{i}^{\prime}$ :

$$
\begin{aligned}
v_{i}\left(v_{1}, w_{1}\right)\left(v_{2}, w_{2}\right)= & v_{i}^{\prime}\left(v_{1} v_{2}\right)=v_{i}^{\prime \prime}\left(w_{1} w_{2}\right) \\
= & \alpha_{2}\left(w_{1}\right) \wedge \alpha_{2}\left(w_{2}\right) \\
= & \left(\alpha_{1}\left(v_{1}\right) \vee \alpha_{2}\left(w_{1}\right)\right) \\
& \wedge\left(\alpha_{1}\left(v_{2}\right) \vee \alpha_{2}\left(w_{2}\right)\right) \\
= & \alpha\left(v_{1}, w_{1}\right) \wedge \alpha\left(v_{2}, w_{2}\right) .
\end{aligned}
$$

Case 2: $v_{1}=v_{2}, w_{1} w_{2} \in R_{i}^{\prime \prime}$ :

$$
\begin{aligned}
v_{i}\left(v_{1}, w_{1}\right)\left(v_{2}, w_{2}\right) & =\alpha_{1}\left(v_{1}\right) \vee v_{i}^{\prime \prime}\left(w_{1} w_{2}\right) \\
& =v_{i}^{\prime \prime}\left(w_{1} w_{2}\right)=\alpha_{2}\left(w_{1}\right) \wedge \alpha_{2}\left(w_{2}\right) \\
& =\left(\alpha_{1}\left(v_{1}\right) \vee \alpha_{2}\left(w_{1}\right)\right) \wedge\left(\alpha_{1}\left(v_{2}\right) \vee \alpha_{2}\left(w_{2}\right)\right) \\
& =\alpha\left(v_{1}, w_{1}\right) \wedge \alpha\left(v_{2}, w_{2}\right) .
\end{aligned}
$$




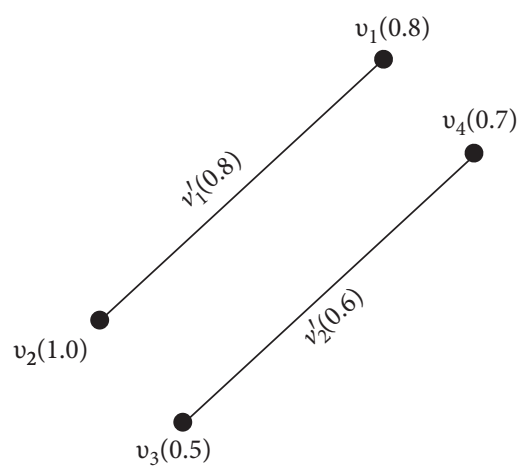

(a)

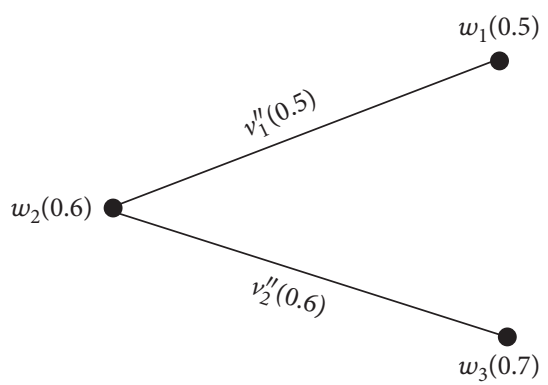

(b)

FIgURe 1: Two fuzzy graph structures. (a) $G_{1}=\left(\alpha_{1}, v_{1}^{\prime}, v_{2}^{\prime}\right)$. (b) $G_{2}=\left(\alpha_{2}, v_{1}^{\prime \prime}, v_{2}^{\prime \prime}\right)$.
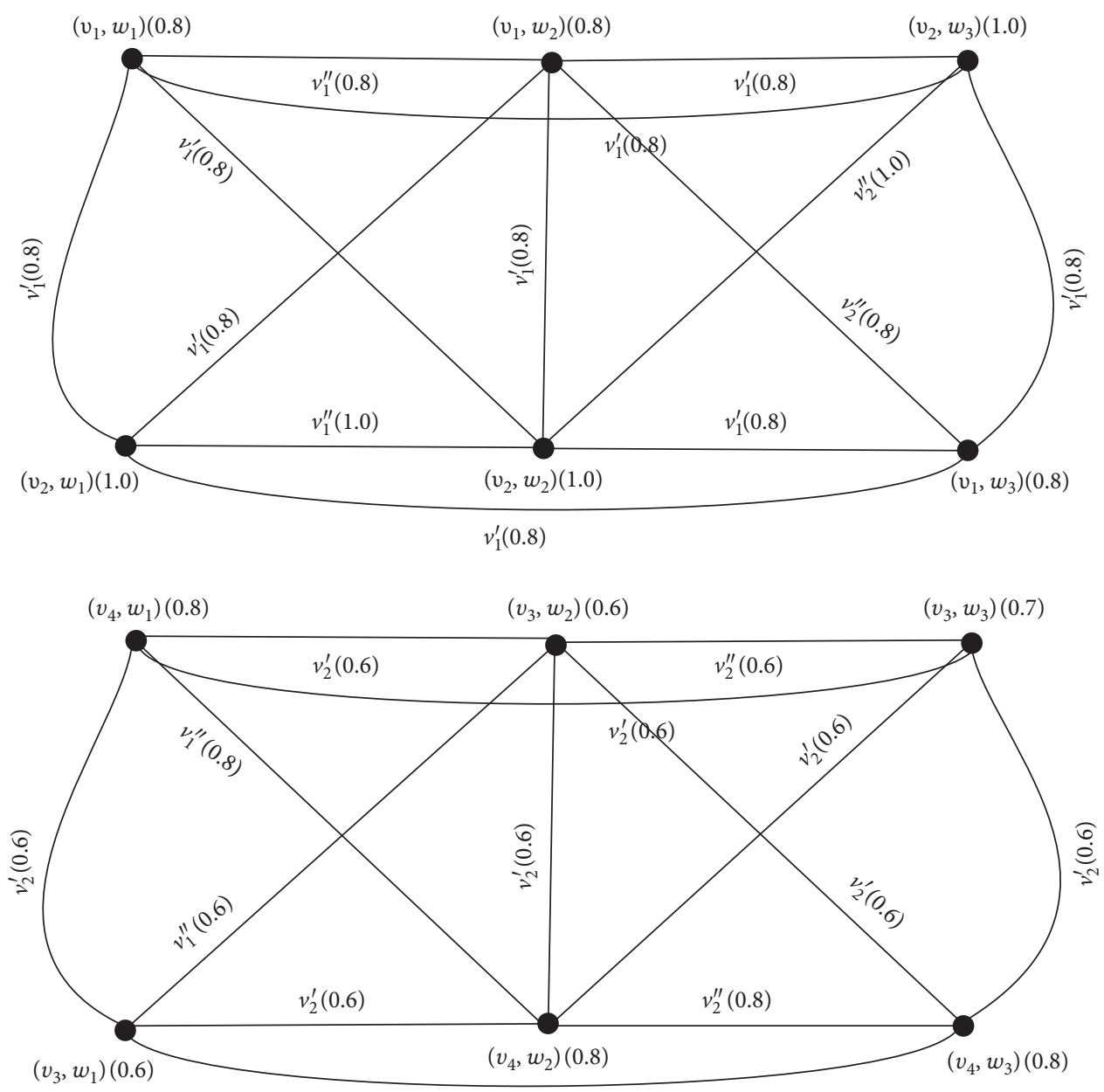

Figure 2: Lexicographic max-product of two FGSs.

Thus, $v_{i}\left(v_{1}, w_{1}\right)\left(v_{2}, w_{2}\right)=\alpha\left(v_{1}, w_{1}\right) \wedge \alpha\left(v_{2}, w_{2}\right)$ for all edges. FGS.

Hence, $\widehat{G}_{1}\left[\widehat{G}_{2}\right]_{\max }=\left(\alpha, v_{1}, v_{2}, \ldots, v_{n}\right)$ is an effective

Example 2. Consider two FGSs $\widehat{G}_{1}=\left(\alpha_{1}, v_{1}^{\prime}, v_{2}^{\prime}\right)$ and $\widehat{G}_{2}=\left(\alpha_{2}, v_{1}^{\prime \prime}, v_{2}^{\prime \prime}\right)$, as shown in Figure 3 .

Figure 3 shows that $v_{1}^{\prime}\left(v_{1} v_{2}\right)=\alpha_{1}\left(v_{1}\right) \wedge \alpha_{1}\left(v_{2}\right)=$ $0.6 \wedge 0.6=0.6, \quad v_{2}^{\prime}\left(v_{3} v_{4}\right)=\alpha_{1}\left(v_{3}\right) \wedge \alpha_{1}\left(v_{4}\right)=0.6 \wedge 0.6=0.6$, $\nu_{1}^{\prime \prime}\left(w_{1} w_{2}\right)=\alpha_{2}\left(w_{1}\right) \wedge \alpha_{2}\left(w_{2}\right)=0.6 \wedge 0.6=0.6, \quad$ and $v_{2}^{\prime \prime}\left(w_{2} w_{3}\right)=\alpha_{2}\left(w_{2}\right) \wedge \alpha_{2}\left(w_{3}\right)=0.6 \wedge 0.6=0.6$. Hence, $\widehat{G}_{1}=$ $\left(\alpha_{1}, v_{1}^{\prime}, v_{2}^{\prime}\right)$ and $\widehat{G}_{2}=\left(\alpha_{2}, v_{1}^{\prime \prime}, v_{2}^{\prime \prime}\right)$ are effective FGSs. Moreover, $\alpha_{1}=\alpha_{2}$ and $\nu_{i}^{\prime}$ and $\nu_{i}^{\prime \prime}, i=1,2$, are constant functions having the same value, i.e., 0.6. The lexicographic-max product of $\widehat{G}_{1}=\left(\alpha_{1}, v_{1}^{\prime}, v_{2}^{\prime}\right)$ and $\widehat{G}_{2}=\left(\alpha_{2}, v_{1}^{\prime \prime}, v_{2}^{\prime \prime}\right)$ is shown in Figure 4.

It is clear from Figure 4 that 


$$
\begin{aligned}
& v_{1}^{\prime}\left(\left(v_{1}, w_{1}\right)\left(v_{2}, w_{1}\right)\right)=\alpha\left(v_{1}, w_{1}\right) \wedge \alpha\left(v_{2}, w_{1}\right)=0.6 \wedge 0.6=0.6, \\
& v_{1}\left(\left(v_{1}, w_{1}\right)\left(v_{1}, w_{2}\right)\right)=\alpha\left(v_{1}, w_{1}\right) \wedge \alpha\left(v_{1}, w_{2}\right)=0.6 \wedge 0.6=0.6, \\
& v_{1}^{\prime}\left(\left(v_{1}, w_{1}\right)\left(v_{2}, w_{2}\right)\right)=\alpha\left(v_{1}, w_{1}\right) \wedge \alpha\left(v_{2}, w_{2}\right)=0.6 \wedge 0.6=0.6, \\
& v_{1}^{\prime}\left(\left(v_{1}, w_{1}\right)\left(v_{2}, w_{3}\right)\right)=\alpha\left(v_{1}, w_{1}\right) \wedge \alpha\left(v_{2}, w_{3}\right)=0.6 \wedge 0.6=0.6, \\
& " v_{1}\left(\left(v_{4}, w_{1}\right)\left(v_{4}, w_{2}\right)\right)=\alpha\left(v_{4}, w_{1}\right) \wedge \alpha\left(v_{4}, w_{2}\right)=0.6 \wedge 0.6=0.6, \\
& v_{2}^{\prime}\left(\left(v_{4}, w_{1}\right)\left(v_{3}, w_{1}\right)\right)=\alpha\left(v_{4}, w_{1}\right) \wedge \alpha\left(v_{3}, w_{1}\right)=0.6 \wedge 0.6=0.6, \\
& v_{2}^{\prime}\left(\left(v_{4}, w_{1}\right)\left(v_{3}, w_{2}\right)\right)=\alpha\left(v_{4}, w_{1}\right) \wedge \alpha\left(v_{3}, w_{2}\right)=0.6 \wedge 0.6=0.6, \\
& v_{2}^{\prime}\left(\left(v_{4}, w_{1}\right)\left(v_{3}, w_{3}\right)\right)=\alpha\left(v_{4}, w_{1}\right) \wedge \alpha\left(v_{3}, w_{3}\right)=0.6 \wedge 0.6=0.6 .
\end{aligned}
$$

Similarly, membership values of all other edges are calculated. Hence, $\widehat{G}_{1}\left[\widehat{G}_{2}\right]_{\max }$ shown in Figure 4 is an effective FGS.

Theorem 2. If $\widehat{G}_{1}$ and $\widehat{G}_{2}$ are two complete FGSs such that $\alpha_{2} \geq \alpha_{1}$ and $v_{i}^{\prime}$ and $v_{i}^{\prime \prime}$ are functions having a constant value, then the lexicographic-max product of $\widehat{G}_{1}$ and $\widehat{G}_{2}$ is a complete FGS.

Proof. The proof is similar to the proof of Theorem 1.

Theorem 3. The lexicographic-max product $\widehat{G}_{1}\left[\widehat{G}_{2}\right]_{\max }$ of two connected FGSs $\widehat{G}_{1}$ and $\widehat{G}_{2}$ is a connected FGS if and only if $\widehat{G}_{1}$ is a connected FGS.

Proof. Assume that $\widehat{G}_{1}$ is a connected FGS. According to the definition of the lexicographic-max product $\widehat{G}_{1}\left[\widehat{G}_{2}\right]_{\max }$, the number of copies of $\widehat{G}_{1}$ is equal to the number of vertices of $\widehat{G}_{2}$; that is, for each vertex of $\widehat{G}_{2}$, there exists one copy of GS $\widehat{G}_{1}$ in the lexicographic-max product $\widehat{G}_{1}\left[\widehat{G}_{2}\right]_{\max }$. Since $\widehat{G}_{1}$ is connected, $\widehat{G}_{1}\left[\widehat{G}_{2}\right]_{\mathrm{max}}$ is a connected FGS. Conversely, assume that $\widehat{G}_{1}$ and $\widehat{G}_{2}$ are two connected FGSs such that $\widehat{G}_{1}\left[\widehat{G}_{2}\right]_{\text {max }}$ is a connected FGS. To prove the connectedness of FGS $\widehat{G}_{1}$, on contrary, assume that $\widehat{G}_{1}$ is not connected. Then, there exist at least two vertices of $\widehat{G}_{1}$ having no path connecting them. Since $\widehat{G}_{1}\left[\widehat{G}_{2}\right]_{\max }$ is connected, then at least one path must exist between any two elements of $V_{1} \times V_{2}$. Thus, there will be at least one path connecting the elements of $\widehat{G}_{1}$. This provides a contradiction. Hence, $\widehat{G}_{1}$ is a connected FGS.

Theorem 4. The number of connected components in the lexicographic-max product $\widehat{G}_{1}\left[\widehat{G}_{2}\right]_{\max }$ of two $F G S s \widehat{G}_{1}$ and $\widehat{G}_{2}$ is equal to the number of components of $\widehat{G}_{1}$.

Proof. Let $\widehat{G}_{1}$ be a connected FGS and $\widehat{G}_{2}$ be FGS. Then, according to Theorem 3, the lexicographic-max product $\widehat{G}_{1}\left[\widehat{G}_{2}\right]_{\max }$ is a connected FGS. This implies that both $\widehat{G}_{1}$ and $\widehat{G}_{1}\left[\widehat{G}_{2}\right]_{\max }$ are connected. Suppose that $\widehat{G}_{1}$ is not a connected FGS having ' $m$ ' distinct connected components. Vertices of $\widehat{G}_{1}$ are renamed such that $\left\{v_{1}, v_{2}, \ldots, v_{k 1}\right\},\left\{v_{k 1+1}, v_{k 1+2}, \ldots\right.$, $\left.v_{k 2}\right\}, \ldots,\left\{v_{k m+1}, v_{k m+2}, \ldots, v_{k m+n}\right\}$ are " $m$ " distinct connected components of $\widehat{G}_{1}$. If $\left\{w_{1}, w_{2}, \ldots, w_{n}\right\}$ are vertices of $\widehat{G}_{2}$, then for an arbitrary vertex of $\widehat{G}_{2}$, there must exist one version of each connected component of $\widehat{G}_{1}$ in the

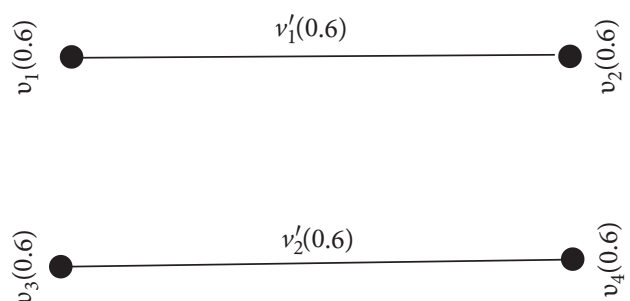

(a)

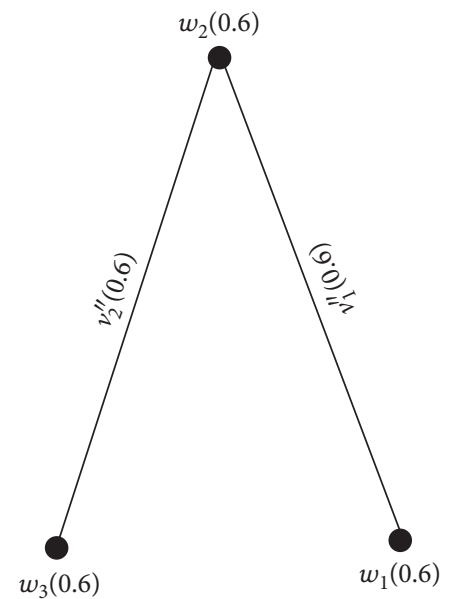

(b)

FIgURE 3: Two fuzzy graph structures. (a) $G_{1}=\left(\alpha_{1}, v_{1}^{\prime}, v_{2}^{\prime}\right)$. (b) $G_{2}=\left(\alpha_{2}, v_{1}^{\prime \prime}, v_{2}^{\prime \prime}\right)$.

lexicographic-max product $\widehat{G}_{1}\left[\widehat{G}_{2}\right]_{\max }$. There is no edge among all these components. Corresponding to each edge between $v_{1} w_{i}$ and $v_{k 1+1} w_{i}$, there must be an edge between vertex $v_{1}$ and $v_{k 1+1}$ of $\widehat{G}_{1}$ which gives a contradiction. Thus, each component of the lexicographic-max product $\widehat{G}_{1}\left[\widehat{G}_{2}\right]_{\max }$ is distinct from others.

Definition 4. In the lexicographic-max product $\widehat{G}_{1}\left[\widehat{G}_{2}\right]_{\max }=$ $\left(\alpha, v_{1}, v_{2}, \ldots, v_{n}\right)$ of FGSs $\widehat{G}_{1}=\left(\alpha_{1}, v_{1}^{\prime}, v_{2}^{\prime}, \ldots, v_{n}^{\prime}\right)$ and $\widehat{G}_{2}=\left(\alpha_{2}, v_{1}^{\prime \prime}, v_{2}^{\prime \prime}, \ldots, v_{n}^{\prime \prime}\right)$, the degree of a vertex is defined as

$$
\begin{aligned}
d_{\widehat{G}_{1}\left[\widehat{G}_{2}\right]_{\max }}\left(v_{i}, w_{j}\right)= & \sum_{v_{i} v_{k} \in R_{i}^{\prime} w_{l} \in V_{2}} v_{i}^{\prime}\left(v_{i} v_{k}\right) \\
& +\sum_{w_{j} w_{l} \in R_{j}^{\prime \prime}, v_{i}=v_{k}}\left(v_{j}^{\prime \prime}\left(w_{j} w_{l}\right) \vee \alpha_{1}\left(v_{i}\right)\right) .
\end{aligned}
$$

$v_{i}$-the degree of a vertex in the lexicographic-max product $\widehat{G}_{1}\left[\widehat{G}_{2}\right]_{\max }$ is defined as

$$
\begin{aligned}
v_{i}-d_{\widehat{G}_{1}\left[\widehat{G}_{2}\right]_{\max }}\left(v_{i}, w_{j}\right)= & \sum_{v_{i} v_{k} \in R_{i}^{\prime} w_{l} \in V_{2}} v_{i}^{\prime}\left(v_{i} v_{k}\right) \\
& +\sum_{w_{j} w_{l} \in R_{i}^{\prime \prime}, v_{i}=v_{k}}\left(v_{i}^{\prime \prime}\left(w_{j} w_{l}\right) \vee \alpha_{1}\left(v_{i}\right)\right) .
\end{aligned}
$$



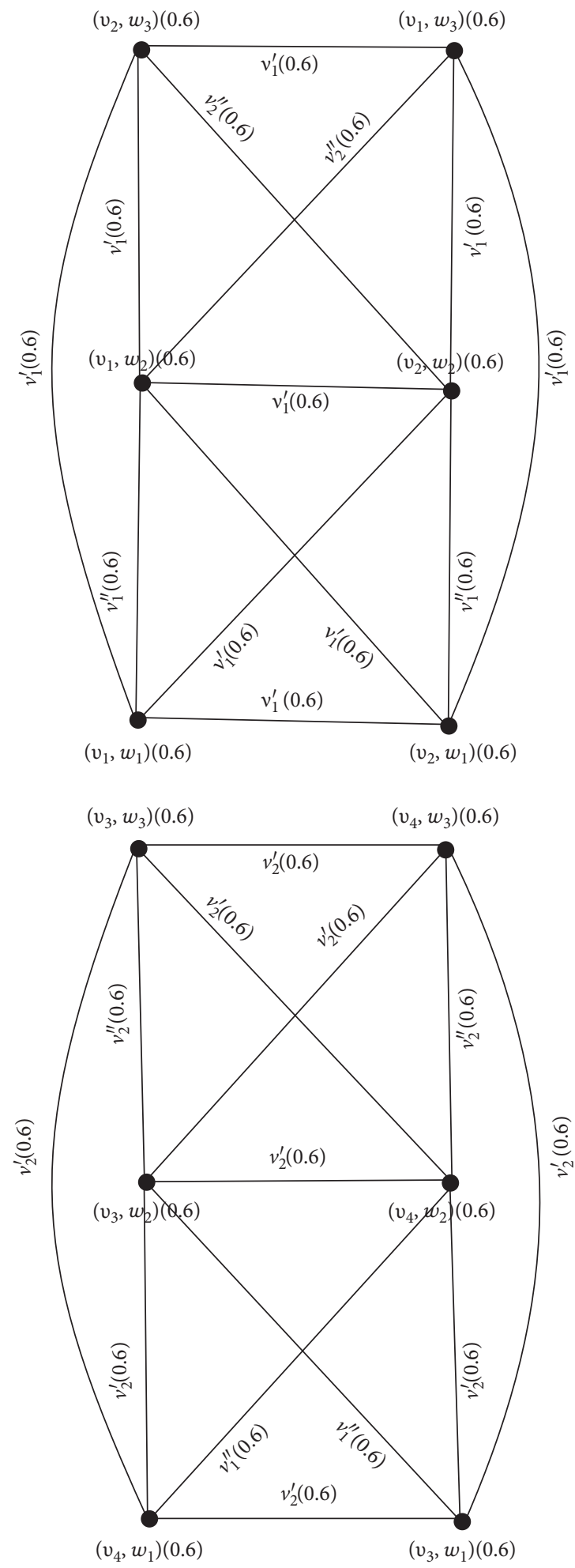

FIGURE 4: Lexicographic-max product of $G_{1}=\left(\alpha_{1}, v_{1}^{\prime}, v_{2}^{\prime}\right)$ and $G_{2}=\left(\alpha_{2}, v_{1}^{\prime \prime}, v_{2}^{\prime \prime}\right)$.

Example 3. Consider two FGSs $\widehat{G}_{1}=\left(\alpha_{1}, \mu_{1}^{\prime}, \mu_{2}^{\prime}\right)$ and $\widehat{G}_{2}=\left(\alpha_{2}, \mu_{1}^{\prime \prime}, \mu_{2}^{\prime \prime}\right)$, which are shown in Figure 5 .

The lexicographic-max product of FGSs $\widehat{G}_{1}$ and $\widehat{G}_{2}$ is shown in Figure 6.

To compute the degrees of all vertices in the lexicographic-max product, the following formula is utilized:

$$
\begin{aligned}
d_{\widehat{G}_{1}\left[\widehat{G}_{2}\right]_{\max }}\left(v_{i}, w_{j}\right)= & \sum_{v_{i} v_{k} \in R_{p}^{\prime} w_{l} \in V_{2}} v_{i}^{\prime}\left(v_{i} v_{k}\right) \\
& +\sum_{w_{j} w_{l} \in R_{j}^{\prime \prime} v_{i}=v_{k}}\left(v_{j}^{\prime \prime}\left(w_{j} w_{l}\right) \vee \alpha_{1}\left(v_{i}\right)\right),
\end{aligned}
$$



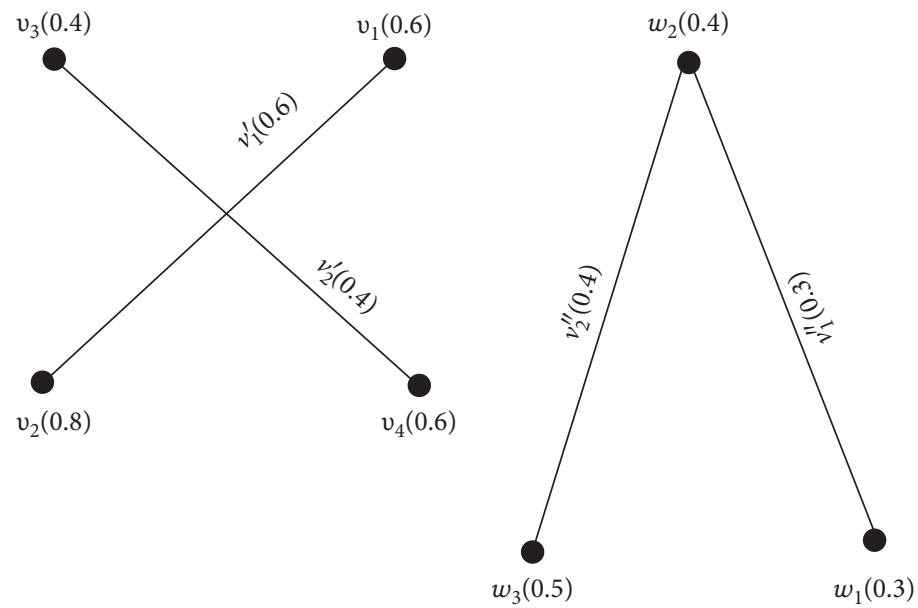

(a)

(b)

FIgURe 5: Two fuzzy graph structures. (a) $G_{1}=\left(\alpha_{1}, v_{1}^{\prime}, v_{2}^{\prime}\right)$. (b) $G_{2}=\left(\alpha_{2}, v_{1}^{\prime \prime}, v_{2}^{\prime \prime}\right)$.
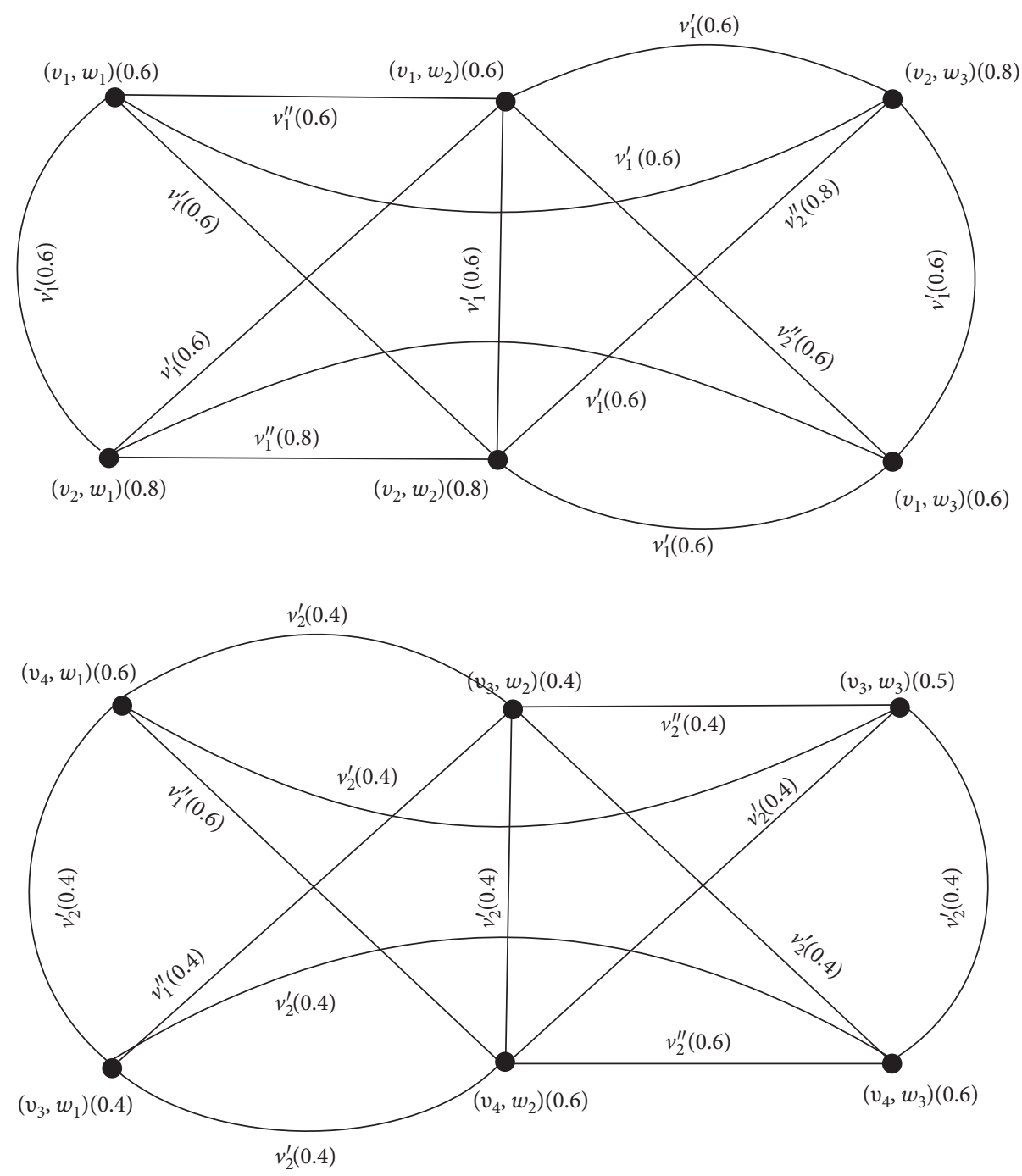

Figure 6: Lexicographic max-product of two FGSs. 


$$
\begin{aligned}
& d_{\widehat{G}_{1}\left[\widehat{G}_{2}\right]_{\max }}\left(v_{1}, w_{1}\right)=v_{1}^{\prime}\left(v_{1} v_{2}\right)+v_{1}^{\prime}\left(v_{1} v_{2}\right) \\
& +v_{1}^{\prime}\left(v_{1} v_{2}\right)+\left(v_{1}^{\prime \prime}\left(w_{1} w_{2}\right) \vee \alpha_{1}\left(v_{1}\right)\right) \\
& =0.6+0.6+0.6+(0.3 \vee 0.6) \\
& =1.8+0.6 \\
& =2.4 \text {, } \\
& d_{\widehat{G}_{1}\left[\widehat{G}_{2}\right]_{\max }}\left(v_{2}, w_{1}\right)=v_{1}^{\prime}\left(v_{2} v_{1}\right)+v_{1}^{\prime}\left(v_{2} v_{1}\right)+v_{1}^{\prime}\left(v_{2} v_{1}\right) \\
& +\left(v_{1}^{\prime \prime}\left(w_{1} w_{2}\right) \vee \alpha_{1}\left(v_{2}\right)\right) \\
& =0.6+0.6+0.6+(0.3 \vee 0.8) \\
& =1.8+0.8 \\
& =2.6 \text {, } \\
& d_{\widehat{G}_{1}\left[\widehat{G}_{2}\right]_{\max }}\left(v_{1}, w_{3}\right)=v_{1}^{\prime}\left(v_{1} v_{2}\right)+v_{1}^{\prime}\left(v_{1} v_{2}\right)+v_{1}^{\prime}\left(v_{1} v_{2}\right) \\
& +\left(v_{2}^{\prime \prime}\left(w_{3} w_{2}\right) \vee \alpha_{1}\left(v_{1}\right)\right) \\
& =0.6+0.6+0.6+(0.4 \vee 0.6) \\
& =1.8+0.6 \\
& =2.4 \text {, } \\
& d_{\widehat{G}_{1}\left[\widehat{G}_{2}\right]_{\max }}\left(v_{2}, w_{3}\right)=v_{1}^{\prime}\left(v_{2} v_{1}\right)+v_{1}^{\prime}\left(v_{2} v_{1}\right)+v_{1}^{\prime}\left(v_{2} v_{1}\right) \\
& +\left(v_{2}^{\prime \prime}\left(w_{3} w_{2}\right) \vee \alpha_{1}\left(v_{2}\right)\right) \\
& =0.6+0.6+0.6+(0.4 \vee 0.8) \\
& =1.8+0.8 \\
& =2.6 \text {, } \\
& d_{\widehat{G}_{1}\left[\widehat{G}_{2}\right]_{\max }}\left(v_{4}, w_{1}\right)=v_{2}^{\prime}\left(v_{4} v_{3}\right)+v_{2}^{\prime}\left(v_{4} v_{3}\right)+v_{2}^{\prime}\left(v_{4} v_{3}\right) \\
& +\left(v_{1}^{\prime \prime}\left(w_{1} w_{2}\right) \vee \alpha_{1}\left(v_{4}\right)\right) \\
& =0.4+0.4+0.4+(0.3 \vee 0.6) \\
& =1.2+0.6 \\
& =1.8 \text {, } \\
& d_{\widehat{G}_{1}\left[\widehat{G}_{2}\right]_{\max }}\left(v_{3}, w_{1}\right)=v_{2}^{\prime}\left(v_{3} v_{4}\right)+v_{2}^{\prime}\left(v_{3} v_{4}\right)+v_{2}^{\prime}\left(v_{3} v_{4}\right) \\
& +\left(v_{1}^{\prime \prime}\left(w_{1} w_{2}\right) \vee \alpha_{1}\left(v_{3}\right)\right) \\
& d_{\widehat{G}_{1}\left[\widehat{G}_{2}\right]_{\max }}\left(v_{2}, w_{2}\right)=v_{1}^{\prime}\left(v_{2} v_{1}\right)+v_{1}^{\prime}\left(v_{2} v_{1}\right)+v_{1}^{\prime}\left(v_{2} v_{1}\right) \\
& +\left(v_{1}^{\prime \prime}\left(w_{2} w_{1}\right) \vee \alpha_{1}\left(v_{2}\right)\right) \\
& +\left(v_{2}^{\prime \prime}\left(w_{2} w_{3}\right) \vee \alpha_{1}\left(v_{2}\right)\right) \\
& =0.6+0.6+0.6+(0.3 \vee 0.8)+(0.4 \vee 0.8) \\
& =1.8+0.8+0.8 \\
& =3.4 \text {. } \\
& d_{\widehat{G}_{1}\left[\widehat{G}_{2}\right]_{\max }}\left(v_{3}, w_{2}\right)=v_{2}^{\prime}\left(v_{3} v_{4}\right)+v_{2}^{\prime}\left(v_{3} v_{4}\right)+v_{2}^{\prime}\left(v_{3} v_{4}\right) \\
& +\left(v_{1}^{\prime \prime}\left(w_{2} w_{1}\right) \vee \alpha_{1}\left(v_{3}\right)\right) \\
& +\left(v_{1}^{\prime \prime}\left(w_{2} w_{1}\right) \vee \alpha_{1}\left(v_{4}\right)\right) \\
& =0.4+0.4+0.4+(0.3 \vee 0.6)+(0.4 \vee 0.6) \\
& +\left(v_{1}^{\prime \prime}\left(w_{2} w_{1}\right) \vee \alpha_{1}\left(v_{1}\right)\right) \\
& =0.6+0.6+0.6+(0.3 \vee 0.6)+(0.4 \vee 0.6) \\
& =1.8+0.6+0.6 \\
& =3.0 \text {, }
\end{aligned}
$$$$
=0.4+0.4+0.4+(0.3 \vee 0.4)
$$$$
=1.2+0.4
$$$$
=1.6 \text {, }
$$$$
d_{\widehat{G}_{1}\left[\widehat{G}_{2}\right]_{\max }}\left(v_{4}, w_{3}\right)=v_{2}^{\prime}\left(v_{4} v_{3}\right)+v_{2}^{\prime}\left(v_{4} v_{3}\right)+v_{2}^{\prime}\left(v_{4} v_{3}\right)
$$$$
+\left(v_{2}^{\prime \prime}\left(w_{3} w_{2}\right) \vee \alpha_{1}\left(v_{4}\right)\right)
$$$$
=0.4+0.4+0.4+(0.4 \vee 0.6)
$$$$
=1.2+0.6
$$$$
=1.8 \text {, }
$$

$v_{i}$-the degree of the vertex in the lexicographic-max product $\widehat{G}_{1}\left[\widehat{G}_{2}\right]_{\max }$ is given by

$$
\begin{aligned}
v_{i}-d_{\widehat{G}_{1}\left[\widehat{G}_{2}\right]_{\max }}\left(v_{i}, w_{j}\right)= & \sum_{v_{i} v_{k} \in R_{p}^{\prime} w_{l} \in V_{2}} v_{i}^{\prime}\left(v_{i} v_{k}\right) \\
& +\sum_{\substack{w_{j} w_{l} \in k_{j}, v_{i}=v_{k} \\
\text { s. }}}\left(v_{i}^{\prime \prime}\left(w_{j} w_{l}\right) \vee \alpha_{1}\left(v_{i}\right)\right) .
\end{aligned}
$$

Using the above given formula, we will calculate $v_{i}$-the degree of the vertices in $\widehat{G}_{1}\left[\widehat{G}_{2}\right]_{\max }$ as

$$
\begin{aligned}
d_{\widehat{G}_{1}\left[\widehat{G}_{2}\right]_{\max }\left(v_{3}, w_{3}\right)=} & v_{2}^{\prime}\left(v_{3} v_{4}\right)+v_{2}^{\prime}\left(v_{3} v_{4}\right)+v_{2}^{\prime}\left(v_{3} v_{4}\right) \\
& +\left(v_{2}^{\prime \prime}\left(w_{3} w_{2}\right) \vee \alpha_{1}\left(v_{3}\right)\right) \\
= & 0.4+0.4+0.4+(0.4 \vee 0.4) \\
= & 1.2+0.4 \\
= & 1.6
\end{aligned}
$$




$$
\begin{aligned}
v_{1}-d_{\widehat{G}_{1}\left[\widehat{G}_{2}\right]_{\max }\left(v_{1}, w_{1}\right)=} & v_{1}^{\prime}\left(v_{1} v_{2}\right)+v_{1}^{\prime}\left(v_{1} v_{2}\right)+v_{1}^{\prime}\left(v_{1} v_{2}\right) \\
& +\left(v_{1}^{\prime \prime}\left(w_{1} w_{2}\right) \vee \alpha_{1}\left(v_{1}\right)\right) \\
= & 0.6+0.6+0.6+(0.3 \vee 0.6) \\
= & 1.8+0.6 \\
= & 2.4
\end{aligned}
$$

$$
\begin{aligned}
v_{1}-d_{\widehat{G}_{1}\left[\widehat{G}_{2}\right]_{\max }\left(v_{2}, w_{1}\right)=} & v_{1}^{\prime}\left(v_{2} v_{1}\right)+v_{1}^{\prime}\left(v_{2} v_{1}\right)+v_{1}^{\prime}\left(v_{2} v_{1}\right) \\
& +\left(v_{1}^{\prime \prime}\left(w_{1} w_{2}\right) \vee \alpha_{1}\left(v_{2}\right)\right) \\
= & 0.6+0.6+0.6+(0.3 \vee 0.8) \\
= & 1.8+0.8 \\
= & 2.6,
\end{aligned}
$$

$$
\begin{aligned}
v_{1}-d_{\widehat{G}_{1}\left[\widehat{G}_{2}\right]_{\max }}\left(v_{1}, w_{2}\right)= & v_{1}^{\prime}\left(v_{1} v_{2}\right)+v_{1}^{\prime}\left(v_{1} v_{2}\right)+v_{1}^{\prime}\left(v_{1} v_{2}\right) \\
& +\left(v_{1}^{\prime \prime}\left(w_{2} w_{1}\right) \vee \alpha_{1}\left(v_{1}\right)\right) \\
= & 0.6+0.6+0.6+(0.3 \vee 0.6) \\
= & 1.8+0.6 \\
= & 2.4
\end{aligned}
$$

$$
\begin{aligned}
v_{1}-d_{\widehat{G}_{1}\left[\widehat{G}_{2}\right]_{\max }}\left(v_{2}, w_{2}\right)= & v_{1}^{\prime}\left(v_{2} v_{1}\right)+v_{1}^{\prime}\left(v_{2} v_{1}\right)+v_{1}^{\prime}\left(v_{2} v_{1}\right) \\
& +\left(v_{1}^{\prime \prime}\left(w_{2} w_{1}\right) \vee \alpha_{1}\left(v_{2}\right)\right) \\
= & 0.6+0.6+0.6+(0.3 \vee 0.8) \\
= & 1.8+0.8 \\
= & 2.6,
\end{aligned}
$$

$$
\begin{aligned}
v_{2}-d_{\widehat{G}_{1}\left[\widehat{G}_{2}\right]_{\max }\left(v_{4}, w_{3}\right)=} & v_{2}^{\prime}\left(v_{4} v_{3}\right)+v_{2}^{\prime}\left(v_{4} v_{3}\right)+v_{2}^{\prime}\left(v_{4} v_{3}\right) \\
& +\left(v_{2}^{\prime \prime}\left(w_{3} w_{2}\right) \vee \alpha_{1}\left(v_{4}\right)\right) \\
= & 0.4+0.4+0.4+(0.4 \vee 0.6) \\
= & 1.2+0.6 \\
= & 1.8,
\end{aligned}
$$

$$
\begin{aligned}
v_{2}-d_{\widehat{G}_{1}\left[\widehat{G}_{2}\right]_{\max }\left(v_{3}, w_{3}\right)=} & v_{2}^{\prime}\left(v_{3} v_{4}\right)+v_{2}^{\prime}\left(v_{3} v_{4}\right)+v_{2}^{\prime}\left(v_{3} v_{4}\right) \\
& +\left(v_{2}^{\prime \prime}\left(w_{3} w_{2}\right) \vee \alpha_{1}\left(v_{3}\right)\right) \\
= & 0.4+0.4+0.4+(0.4 \vee 0.4) \\
= & 1.2+0.4 \\
= & 1.6
\end{aligned}
$$

$$
\begin{aligned}
v_{2}-d_{\widehat{G}_{1}\left[\widehat{G}_{2}\right]_{\max }\left(v_{3}, w_{2}\right)=} & v_{2}^{\prime}\left(v_{3} v_{4}\right)+v_{2}^{\prime}\left(v_{3} v_{4}\right)+v_{2}^{\prime}\left(v_{3} v_{4}\right) \\
& +\left(v_{2}^{\prime \prime}\left(w_{2} w_{3}\right) \vee \alpha_{1}\left(v_{3}\right)\right) \\
= & 0.4+0.4+0.4+(0.4 \vee 0.4) \\
= & 1.2+0.4 \\
= & 1.6,
\end{aligned}
$$

$$
\begin{aligned}
v_{2}-d_{\widehat{G}_{1}\left[\widehat{G}_{2}\right]_{\max }\left(v_{4}, w_{2}\right)=} & v_{2}^{\prime}\left(v_{4} v_{3}\right)+v_{2}^{\prime}\left(v_{4} v_{3}\right)+v_{2}^{\prime}\left(v_{4} v_{3}\right) \\
& +\left(v_{2}^{\prime \prime}\left(w_{2} w_{3}\right) \vee \alpha_{1}\left(v_{4}\right)\right) \\
= & 0.4+0.4+0.4+(0.4 \vee 0.6) \\
= & 1.2+0.6 \\
= & 1.8
\end{aligned}
$$

$$
\begin{aligned}
v_{1}-d_{\widehat{G}_{1}\left[\widehat{G}_{2}\right]_{\max }}\left(v_{1}, w_{3}\right) & =v_{1}^{\prime}\left(v_{1} v_{2}\right)+v_{1}^{\prime}\left(v_{1} v_{2}\right)+v_{1}^{\prime}\left(v_{1} v_{2}\right) \\
& =0.6+0.6+0.6 \\
& =1.8
\end{aligned}
$$

$$
\begin{aligned}
v_{1}-d_{\widehat{G}_{1}\left[\widehat{G}_{2}\right]_{\max }}\left(v_{2}, w_{3}\right) & =v_{1}^{\prime}\left(v_{2} v_{1}\right)+v_{1}^{\prime}\left(v_{2} v_{1}\right)+v_{1}^{\prime}\left(v_{2} v_{1}\right) \\
& =0.6+0.6+0.6 \\
& =1.8
\end{aligned}
$$

$$
\begin{aligned}
v_{2}-d_{\widehat{G}_{1}\left[\widehat{G}_{2}\right]_{\max }}\left(v_{4}, w_{1}\right) & =v_{2}^{\prime}\left(v_{4} v_{3}\right)+v_{2}^{\prime}\left(v_{4} v_{3}\right)+v_{2}^{\prime}\left(v_{4} v_{3}\right) \\
& =0.4+0.4+0.4 \\
& =1.2,
\end{aligned}
$$

$$
\begin{aligned}
v_{2}-d_{\widehat{G}_{1}\left[\widehat{G}_{2}\right]_{\max }}\left(v_{3}, w_{1}\right) & =v_{2}^{\prime}\left(v_{3} v_{4}\right)+v_{2}^{\prime}\left(v_{3} v_{4}\right)+v_{2}^{\prime}\left(v_{3} v_{4}\right) \\
& =0.4+0.4+0.4 \\
& =1.2
\end{aligned}
$$

$$
\begin{aligned}
& v_{1}-d_{\widehat{G}_{1}\left[\widehat{G}_{2}\right]_{\max }}\left(v_{4}, w_{2}\right)=v_{1}^{\prime \prime}\left(w_{2} w_{1}\right) \vee \alpha_{1}\left(v_{4}\right)=0.3 \vee 0.6=0.6, \\
& v_{1}-d_{\widehat{G}_{1}\left[\widehat{G}_{2}\right]_{\max }}\left(v_{3}, w_{2}\right)=v_{1}^{\prime \prime}\left(w_{2} w_{1}\right) \vee \alpha_{1}\left(v_{3}\right)=0.3 \vee 0.4=0.4, \\
& v_{1}-d_{\widehat{G}_{1}\left[\widehat{G}_{2}\right]_{\max }}\left(v_{3}, w_{1}\right)=v_{1}^{\prime \prime}\left(w_{1} w_{2}\right) \vee \alpha_{1}\left(v_{3}\right)=0.3 \vee 0.4=0.4, \\
& v_{1}-d_{\widehat{G}_{1}\left[\widehat{G}_{2}\right]_{\max }}\left(v_{4}, w_{1}\right)=v_{1}^{\prime \prime}\left(w_{1} w_{2}\right) \vee \alpha_{1}\left(v_{4}\right)=0.3 \vee 0.6=0.6, \\
& v_{2}-d_{\widehat{G}_{1}\left[\widehat{G}_{2}\right]_{\max }}\left(v_{2}, w_{2}\right)=v_{2}^{\prime \prime}\left(w_{2} w_{3}\right) \vee \alpha_{1}\left(v_{2}\right)=0.4 \vee 0.8=0.8, \\
& v_{2}-d_{\widehat{G}_{1}\left[\widehat{G}_{2}\right]_{\max }}\left(v_{1}, w_{2}\right)=v_{2}^{\prime \prime}\left(w_{2} w_{3}\right) \vee \alpha_{1}\left(v_{1}\right)=0.4 \vee 0.6=0.6,
\end{aligned}
$$


$v_{2}-d_{\widehat{G}_{1}\left[\widehat{G}_{2}\right]_{\max }}\left(v_{2}, w_{3}\right)=v_{2}^{\prime \prime}\left(w_{2} w_{3}\right) \vee \alpha_{1}\left(v_{2}\right)=0.4 \vee 0.8=0.8$,

$v_{2}-d_{\widehat{G}_{1}\left[\widehat{G}_{2}\right]_{\max }}\left(v_{1}, w_{3}\right)=v_{2}^{\prime \prime}\left(w_{2} w_{3}\right) \vee \alpha_{1}\left(v_{1}\right)=0.4 \vee 0.6=0.6$.

Theorem 5. If $\widehat{G}_{1}$ and $\widehat{G}_{2}$ are two FGSs such that $\nu_{i}^{\prime \prime} \geq \alpha_{1}$, then the degree of the vertex in the lexicographic-max product $\widehat{G}_{1}\left[\widehat{G}_{2}\right]_{\max }$ of FGSs $\widehat{G}_{1}$ and $\widehat{G}_{2}$ is given by $d_{\widehat{G}_{1}\left[\widehat{G}_{2}\right]_{\max }}\left(v_{i}\right.$, $\left.w_{j}\right)=d_{\widehat{G}_{2}}\left(w_{j}\right)+d_{\widehat{G}_{1}}\left(v_{i}\right)\left|V_{2}\right|$.

Proof. Let $\widehat{G}_{1}$ and $\widehat{G}_{2}$ be two FGSs such that $\mu_{i}^{\prime \prime} \geq \alpha_{1}$, then $\mu_{i}^{\prime \prime} \vee \alpha_{1}=\mu_{i}^{\prime \prime}$, and then the degree of the vertex in $\widehat{G}_{1}\left[\widehat{G}_{2}\right]_{\max }$ (lexicographic-max product) is given by

$$
\begin{aligned}
& d_{\widehat{G}_{1}\left[\widehat{G}_{2}\right]_{\max }\left(v_{i}, v_{j}\right)=} \sum_{v_{i} v_{k} \in R_{p}^{\prime} w_{l} \in V_{2}} \mu_{i}^{\prime}\left(v_{i} v_{k}\right) \\
&+\sum_{w_{j} w_{l} \in R_{j}^{\prime \prime}, v_{i}=v_{k}}\left(\mu_{j}^{\prime \prime}\left(w_{j} w_{l}\right) \vee \alpha_{1}\left(v_{i}\right)\right) \\
&=\left|V_{2}\right| \sum_{v_{i} v_{k} \in R_{i}^{\prime}, w_{j}=w_{l}} \mu_{i}^{\prime}\left(v_{i} v_{k}\right) \\
&+\sum_{w_{j} w_{l} \in R_{j}^{\prime \prime}, v_{i}=v_{k}} \mu_{j}^{\prime \prime}\left(w_{j} w_{l}\right) \\
&=\left|V_{2}\right| d_{\widehat{G}_{1}}\left(v_{i}\right)+d_{\widehat{G}_{2}}\left(w_{j}\right) .
\end{aligned}
$$

Theorem 6. If $\widehat{G}_{1}$ and $\widehat{G}_{2}$ are two FGSs such that $\nu_{i}^{\prime \prime} \geq \alpha_{1}$, then $v_{i}$-the degree of any vertex in the lexicographic-max product $\widehat{G}_{1}\left[\widehat{G}_{2}\right]_{\text {max }}$ of FGSs $\widehat{G}_{1}$ and $\widehat{G}_{2}$ is given by $v_{i}-d_{\widehat{G}_{1}}\left[\widehat{G}_{2}\right]_{\max }$ $\left(v_{i}, w_{j}\right)=\left[v_{i}^{\prime \prime}-d_{\widehat{G}_{2}}\left(w_{j}\right)\right]+\left[v_{i}^{\prime}-d_{\widehat{G}_{1}}\left(v_{i}\right)\right]\left|V_{2}\right|$.

Proof. Let $\widehat{G}_{1}=\left(\alpha_{1}, v_{1}^{\prime}, v_{2}^{\prime}, \ldots, v_{n}^{\prime}\right)$ and $\widehat{G}_{2}=\left(\alpha_{2}, v_{1}^{\prime \prime}\right.$, $\left.v_{2}^{\prime \prime}, \ldots, v_{n}^{\prime \prime}\right)$ be two FGSs such that $v_{i}^{\prime \prime} \geq \alpha_{1}$, then $\nu_{i}^{\prime \prime} \vee \alpha_{1}=v_{i}^{\prime \prime}$, $i=1,2, \ldots, n$. Then, $v_{i}$-the degree of the vertex in $\widehat{G}_{1}\left[\widehat{G}_{2}\right]_{\max }$ (lexicographic-max product) is given as

$$
\begin{aligned}
& v_{i}-d_{\widehat{G}_{1}\left[\widehat{G}_{2}\right]_{\max }\left(v_{i}, w_{j}\right)=} \sum_{w_{j} w_{l} \in R_{i}^{\prime \prime}, v_{i}=v_{k}}\left(v_{i}^{\prime \prime}\left(w_{j} w_{l}\right) \vee \alpha_{1}\left(v_{i}\right)\right) \\
&+\sum_{v_{i} v_{k} \in R_{i}^{\prime}, w_{l} \in V_{2}} v_{i}^{\prime}\left(v_{i} v_{k}\right) \\
&=\sum_{w_{j} w_{l} \in R_{i}^{\prime \prime}, v_{i}=v_{k}} v_{i}^{\prime \prime}\left(w_{j} w_{l}\right) \\
&+\left|V_{2}\right| \sum_{v_{i} v_{k} \in R_{i}^{\prime}, w_{j}=w_{l}} v_{i}^{\prime}\left(v_{i} v_{k}\right) \\
&= {\left[v_{i}^{\prime \prime}-d_{\widehat{G}_{2}}\left(w_{j}\right)\right]+\left[v_{i}^{\prime}-d_{\widehat{G}_{1}}\left(v_{i}\right)\right]\left|V_{2}\right| . }
\end{aligned}
$$

Example 4. Consider two FGSs $\widehat{G}_{1}=\left(\alpha_{1}, \mu_{1}^{\prime}, \mu_{2}^{\prime}\right)$ and $\widehat{G}_{2}=\left(\alpha_{2}, \mu_{1}^{\prime \prime}, \mu_{2}^{\prime \prime}\right)$, shown in Figure 7 .

In Figure $7, \mu_{i}^{\prime \prime} \geq \alpha_{1}$. The lexicographic-max product of $\widehat{G}_{1}=\left(\alpha_{1}, \mu_{1}^{\prime}, \mu_{2}^{\prime}\right)$ and $\widehat{G}_{2}=\left(\alpha_{2}, \mu_{1}^{\prime \prime}, \mu_{2}^{\prime \prime}\right)$ is shown in Figure 8.

Theorem 6 implies the formula to determine the degrees of vertices in the lexicographic-max product as follows:

$$
v_{i}-d_{\widehat{G}_{1}\left[\widehat{G}_{2}\right]_{\max }}\left(v_{i}, w_{j}\right)=\left[\nu_{i}^{\prime \prime}-d_{\widehat{G}_{2}}\left(w_{j}\right)\right]+\left[v_{i}^{\prime}-d_{\widehat{G}_{1}}\left(v_{i}\right)\right]\left|V_{2}\right| \text {, }
$$

$$
\begin{aligned}
v_{1}-d_{\widehat{G}_{1}\left[\widehat{G}_{2}\right]_{\max }}\left(v_{1}, w_{1}\right) & =\left|V_{2}\right|\left[v_{1}^{\prime}-d_{\widehat{G}_{1}}\left(v_{1}\right)\right]+\left[v_{1}^{\prime \prime}-d_{\widehat{G}_{2}}\left(w_{1}\right)\right] \\
& =(3)(0.6)+0.8 \\
& =1.8+0.8 \\
& =2.6
\end{aligned}
$$

$$
\begin{aligned}
v_{2}-d_{\widehat{G}_{1}\left[\widehat{G}_{2}\right]_{\max }}\left(v_{1}, w_{1}\right) & =\left|V_{2}\right|\left[v_{2}^{\prime}-d_{\widehat{G}_{1}}\left(v_{1}\right)\right]+\left[v_{2}^{\prime \prime}-d_{\widehat{G}_{2}}\left(w_{1}\right)\right] \\
& =(3)(0)+0 \\
& =0+0 \\
& =0
\end{aligned}
$$

$$
\begin{aligned}
v_{1}-d_{\widehat{G}_{1}\left[\widehat{G}_{2}\right]_{\max }}\left(v_{1}, w_{2}\right) & =\left|V_{2}\right|\left[v_{1}^{\prime}-d_{\widehat{G}_{1}}\left(v_{1}\right)\right]+\left[v_{1}^{\prime \prime}-d_{\widehat{G}_{2}}\left(w_{2}\right)\right] \\
& =(3)(0.6)+0.8 \\
& =1.8+0.8 \\
& =2.6
\end{aligned}
$$

$$
\begin{aligned}
v_{2}-d_{\widehat{G}_{1}\left[\widehat{G}_{2}\right]_{\max }}\left(v_{1}, w_{2}\right) & =\left|V_{2}\right|\left[v_{2}^{\prime}-d_{\widehat{G}_{1}}\left(v_{1}\right)\right]+\left[v_{2}^{\prime \prime}-d_{\widehat{G}_{2}}\left(w_{2}\right)\right] \\
& =(3)(0)+0.8 \\
& =0+0.8 \\
& =0.8,
\end{aligned}
$$

$$
\begin{aligned}
v_{1}-d_{\widehat{G}_{1}\left[\widehat{G}_{2}\right]_{\max }\left(v_{1}, w_{3}\right)}=\left|V_{2}\right|\left[v_{1}^{\prime}-d_{\widehat{G}_{1}}\left(v_{1}\right)\right]+\left[v_{1}^{\prime \prime}-d_{\widehat{G}_{2}}\left(w_{3}\right)\right] \\
=(3)(0.6)+0 \\
=1.8+0 \\
=1.8
\end{aligned}
$$

$$
\begin{aligned}
v_{2}-d_{\widehat{G}_{1}\left[\widehat{G}_{2}\right]_{\max }}\left(v_{1}, w_{3}\right) & =\left|V_{2}\right|\left[v_{2}^{\prime}-d_{\widehat{G}_{1}}\left(v_{1}\right)\right]+\left[v_{2}^{\prime \prime}-d_{\widehat{G}_{2}}\left(w_{3}\right)\right] \\
& =(3)(0)+0.8 \\
& =0+0.8 \\
& =0.8,
\end{aligned}
$$




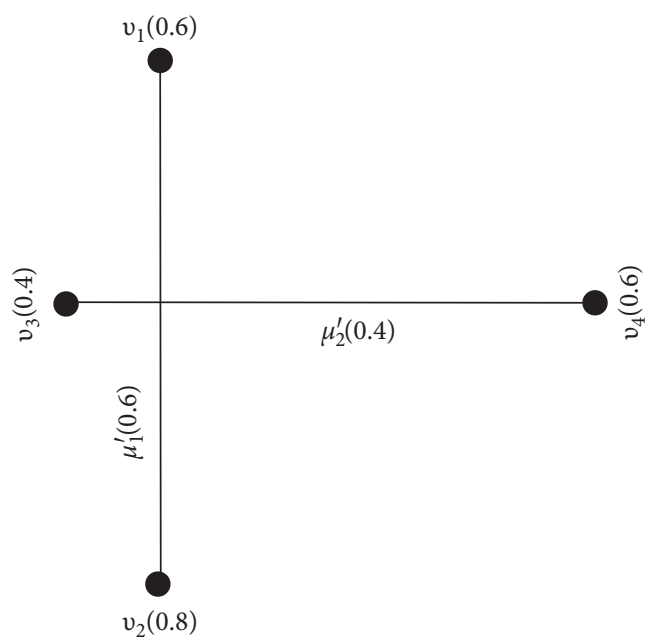

(a)

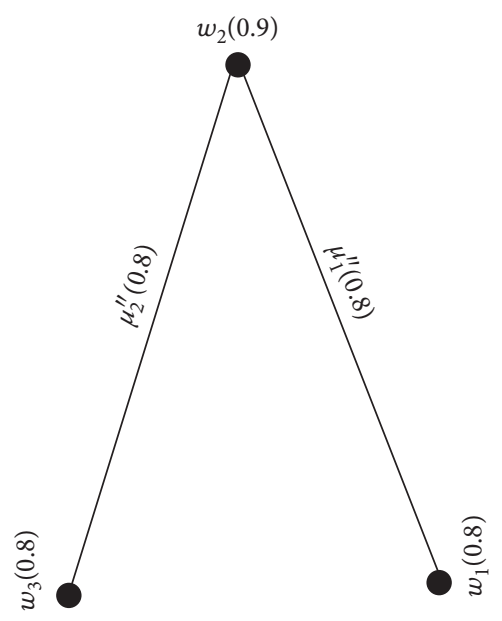

(b)

Figure 7: Two fuzzy graph structures. (a) $G_{1}=\left(\alpha_{1}, \mu_{1}^{\prime}, \mu_{2}^{\prime}\right)$. (b) $G_{2}=\left(\alpha_{2}, \mu_{1}^{\prime \prime}, \mu_{2}^{\prime \prime}\right)$.
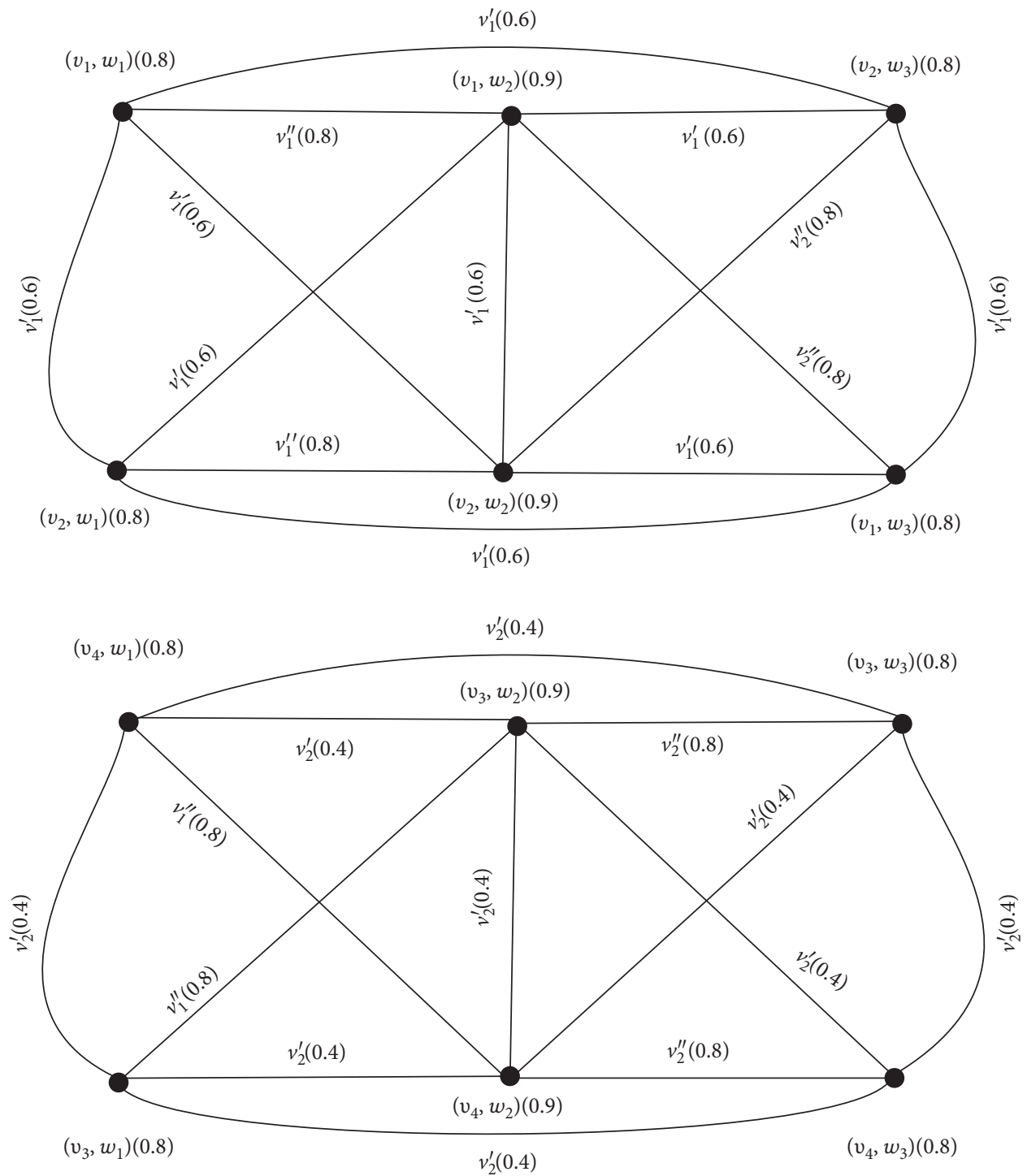

FIgURE 8: Lexicographic-max product of two FGSs. 


$$
\begin{aligned}
v_{1}-d_{\widehat{G}_{1}\left[\widehat{G}_{2}\right]_{\max }}\left(v_{2}, w_{1}\right) & =\left|V_{2}\right|\left[v_{1}^{\prime}-d_{\widehat{G}_{1}}\left(v_{2}\right)\right]+\left[v_{1}^{\prime \prime}-d_{\widehat{G}_{2}}\left(w_{1}\right)\right] \\
& =(3)(0.6)+0.8 \\
& =1.8+0.8 \\
& =2.6
\end{aligned}
$$

$$
\begin{aligned}
v_{2}-d_{\widehat{G}_{1}\left[\widehat{G}_{2}\right]_{\max }}\left(v_{2}, w_{1}\right) & =\left|V_{2}\right|\left[v_{2}^{\prime}-d_{\widehat{G}_{1}}\left(v_{2}\right)\right]+\left[v_{2}^{\prime \prime}-d_{\widehat{G}_{2}}\left(w_{1}\right)\right] \\
& =(3)(0)+0 \\
& =0+0 \\
& =0,
\end{aligned}
$$

$$
\begin{aligned}
v_{1}-d_{\widehat{G}_{1}\left[\widehat{G}_{2}\right]_{\max }}\left(v_{2}, w_{2}\right) & =\left|V_{2}\right|\left[v_{1}^{\prime}-d_{\widehat{G}_{1}}\left(v_{2}\right)\right]+\left[v_{1}^{\prime \prime}-d_{\widehat{G}_{2}}\left(w_{2}\right)\right] \\
& =(3)(0.6)+0.8 \\
& =1.8+0.8 \\
& =2.6
\end{aligned}
$$

$$
\begin{aligned}
v_{2}-d_{\widehat{G}_{1}\left[\widehat{G}_{2}\right]_{\max }}\left(v_{2}, w_{2}\right) & =\left|V_{2}\right|\left[v_{2}^{\prime}-d_{\widehat{G}_{1}}\left(v_{2}\right)\right]+\left[v_{2}^{\prime \prime}-d_{\widehat{G}_{2}}\left(w_{2}\right)\right] \\
& =(3)(0)+0.8 \\
& =0+0.8 \\
& =0.8,
\end{aligned}
$$

$$
\begin{aligned}
& v_{1}-d_{\widehat{G}_{1}\left[\widehat{G}_{2}\right]_{\max }\left(v_{2}, w_{3}\right)}=\left|V_{2}\right|\left[v_{1}^{\prime}-d_{\widehat{G}_{1}}\left(v_{2}\right)\right]+\left[v_{1}^{\prime \prime}-d_{\widehat{G}_{2}}\left(w_{3}\right)\right] \\
&=(3)(0.6)+0 \\
&=1.8+0 \\
&=1.8,
\end{aligned}
$$

$$
\begin{aligned}
v_{2}-d_{\widehat{G}_{1}\left[\widehat{G}_{2}\right]_{\max }}\left(v_{2}, w_{3}\right) & =\left|V_{2}\right|\left[v_{2}^{\prime}-d_{\widehat{G}_{1}}\left(v_{2}\right)\right]+\left[v_{2}^{\prime \prime}-d_{\widehat{G}_{2}}\left(w_{3}\right)\right] \\
& =(3)(0)+0.8 \\
& =(3)(0)+0.8 \\
& =0.8,
\end{aligned}
$$

$$
\begin{aligned}
v_{1}-d_{\widehat{G}_{1}\left[\widehat{G}_{2}\right]_{\max }}\left(v_{3}, w_{1}\right) & =\left|V_{2}\right|\left[v_{1}^{\prime}-d_{\widehat{G}_{1}}\left(v_{3}\right)\right]+\left[v_{1}^{\prime \prime}-d_{\widehat{G}_{2}}\left(w_{1}\right)\right] \\
& =(3)(0)+0.8 \\
& =0+0.8 \\
& =0.8,
\end{aligned}
$$

$$
\begin{aligned}
v_{2}-d_{\widehat{G}_{1}\left[\widehat{G}_{2}\right]_{\max }}\left(v_{3}, w_{1}\right) & =\left|V_{2}\right|\left[v_{2}^{\prime}-d_{\widehat{G}_{1}}\left(v_{3}\right)\right]+\left[v_{2}^{\prime \prime}-d_{\widehat{G}_{2}}\left(w_{1}\right)\right] \\
& =(3)(0.4)+0 \\
& =1.2+0 \\
& =1.2
\end{aligned}
$$

$$
\begin{aligned}
v_{1}-d_{\widehat{G}_{1}\left[\widehat{G}_{2}\right]_{\max }}\left(v_{3}, w_{2}\right) & =\left|V_{2}\right|\left[v_{1}^{\prime}-d_{\widehat{G}_{1}}\left(v_{3}\right)\right]+\left[v_{1}^{\prime \prime}-d_{\widehat{G}_{2}}\left(w_{2}\right)\right] \\
& =(3)(0)+0.8 \\
& =0+0.8 \\
& =0
\end{aligned}
$$

$$
\begin{aligned}
& v_{2}-d_{\widehat{G}_{1}\left[\widehat{G}_{2}\right]_{\max }\left(v_{3}, w_{2}\right)}=\left|V_{2}\right|\left[v_{2}^{\prime}-d_{\widehat{G}_{1}}\left(v_{3}\right)\right]+\left[v_{2}^{\prime \prime}-d_{\widehat{G}_{2}}\left(w_{2}\right)\right] \\
&=(3)(0.4)+0.8 \\
&=1.2+0.8 \\
&=2.0
\end{aligned}
$$

$$
\begin{aligned}
v_{1}-d_{\widehat{G}_{1}\left[\widehat{G}_{2}\right]_{\max }}\left(v_{3}, w_{3}\right) & =\left|V_{2}\right|\left[v_{1}^{\prime}-d_{\widehat{G}_{1}}\left(v_{3}\right)\right]+\left[v_{1}^{\prime \prime}-d_{\widehat{G}_{2}}\left(w_{3}\right)\right] \\
& =(3)(0)+0 \\
& =0+0 \\
& =0
\end{aligned}
$$

$$
\begin{aligned}
v_{2}-d_{\widehat{G}_{1}\left[\widehat{G}_{2}\right]_{\max }}\left(v_{3}, w_{3}\right) & =\left|V_{2}\right|\left[v_{2}^{\prime}-d_{\widehat{G}_{1}}\left(v_{3}\right)\right]+\left[v_{2}^{\prime \prime}-d_{\widehat{G}_{2}}\left(w_{3}\right)\right] \\
& =(3)(0.4)+0.8 \\
& =1.2+0.8 \\
& =2.0
\end{aligned}
$$

$$
\begin{aligned}
v_{1}-d_{\widehat{G}_{1}\left[\widehat{G}_{2}\right]_{\max }}\left(v_{4}, w_{1}\right) & =\left|V_{2}\right|\left[v_{1}^{\prime}-d_{\widehat{G}_{1}}\left(v_{4}\right)\right]+\left[v_{1}^{\prime \prime}-d_{\widehat{G}_{2}}\left(w_{1}\right)\right] \\
& =(3)(0)+0.8 \\
& =0+0.8 \\
& =0.8,
\end{aligned}
$$

$$
\begin{aligned}
& v_{2}-d_{\widehat{G}_{1}\left[\widehat{G}_{2}\right]_{\max }\left(v_{4}, w_{1}\right)}=\left|V_{2}\right|\left[v_{2}^{\prime}-d_{\widehat{G}_{1}}\left(v_{4}\right)\right]+\left[v_{2}^{\prime \prime}-d_{\widehat{G}_{2}}\left(w_{1}\right)\right] \\
&=(3)(0.4)+0 \\
&=1.2+0 \\
&=1.2
\end{aligned}
$$

(49) 


$$
\begin{aligned}
v_{1}-d_{\widehat{G}_{1}}\left[\widehat{G}_{2}\right]_{\max }\left(v_{4}, w_{2}\right) & =\left|V_{2}\right|\left[v_{1}^{\prime}-d_{\widehat{G}_{1}}\left(v_{4}\right)\right]+\left[v_{1}^{\prime \prime}-d_{\widehat{G}_{2}}\left(w_{2}\right)\right] \\
& =(3)(0)+0.8 \\
& =0+0.8 \\
& =0.8,
\end{aligned}
$$

$$
\begin{aligned}
v_{2}-d_{\widehat{G}_{1}\left[\widehat{G}_{2}\right]_{\max }}\left(v_{4}, w_{2}\right) & =\left|V_{2}\right|\left[v_{2}^{\prime}-d_{\widehat{G}_{1}}\left(v_{4}\right)\right]+\left[v_{2}^{\prime \prime}-d_{\widehat{G}_{2}}\left(w_{2}\right)\right] \\
& =(3)(0.4)+0.8 \\
& =1.2+0.8 \\
& =2.0,
\end{aligned}
$$

$$
\begin{aligned}
v_{1}-d_{\widehat{G}_{1}\left[\widehat{G}_{2}\right]_{\max }}\left(v_{4}, w_{3}\right) & =\left|V_{2}\right|\left[v_{1}^{\prime}-d_{\widehat{G}_{1}}\left(v_{4}\right)\right]+\left[v_{1}^{\prime \prime}-d_{\widehat{G}_{2}}\left(w_{3}\right)\right] \\
& =(3)(0)+0 \\
& =0+0 \\
& =0
\end{aligned}
$$

$$
\begin{aligned}
v_{2}-d_{\widehat{G}_{1}\left[\widehat{G}_{2}\right]_{\max }}\left(v_{4}, w_{3}\right) & =\left|V_{2}\right|\left[v_{2}^{\prime}-d_{\widehat{G}_{1}}\left(v_{4}\right)\right]+\left[v_{2}^{\prime \prime}-d_{\widehat{G}_{2}}\left(w_{3}\right)\right] \\
& =(3)(0.4)+0.8 \\
& =1.2+0.8 \\
& =2.0
\end{aligned}
$$

Through direct calculations,

$$
\begin{aligned}
& v_{1}-d_{\widehat{G}_{1}\left[\widehat{G}_{2}\right]_{\max }}\left(v_{4}, w_{1}\right)=0.8, v_{2}-d_{\widehat{G}_{1}\left[\widehat{G}_{2}\right]_{\max }}\left(v_{2}, w_{3}\right)=0.8 \text {, } \\
& v_{1}-d_{\widehat{G}_{1}\left[\widehat{G}_{2}\right]_{\max }}\left(v_{3}, w_{1}\right)=0.8, v_{2}-d_{\widehat{G}_{1}}\left[\widehat{G}_{2}\right]_{\max }\left(v_{1}, w_{3}\right)=0.8 \text {, } \\
& v_{1}-d_{\widehat{G}_{1}\left[\widehat{G}_{2}\right]_{\max }}\left(v_{3}, w_{2}\right)=0.8, v_{2}-d_{\widehat{G}_{1}}\left[\widehat{G}_{2}\right]_{\max }\left(v_{2}, w_{2}\right)=0.8 \text {, } \\
& v_{1}-d_{\widehat{G}_{1}}\left[\widehat{G}_{2}\right]_{\max }\left(v_{4}, w_{2}\right)=0.8, v_{2}-d_{\widehat{G}_{1}}\left[\widehat{G}_{2}\right]_{\max }\left(v_{1}, w_{2}\right)=0.8 \text {, } \\
& v_{1}-d_{\widehat{G}_{1}\left[\widehat{G}_{2}\right]_{\max }}\left(v_{4}, w_{3}\right)=0, v_{2}-d_{\widehat{G}_{1}\left[\widehat{G}_{2}\right]_{\max }}\left(v_{1}, w_{1}\right)=0 \text {, } \\
& v_{1}-d_{\widehat{G}_{1}}\left[\widehat{G}_{2}\right]_{\max }\left(v_{3}, w_{3}\right)=0, v_{2}-d_{\widehat{G}_{1}}\left[\widehat{G}_{2}\right]_{\max }\left(v_{2}, w_{1}\right)=0, \\
& v_{1}-d_{\widehat{G}_{1}}\left[\widehat{G}_{2}\right]_{\max }\left(v_{1}, w_{1}\right)=0.6+0.6+0.6+0.8=2.6 \text {, } \\
& v_{2}-d_{\widehat{G}_{1}}\left[\widehat{G}_{2}\right]_{\max }\left(v_{4}, w_{3}\right)=0.4+0.4+0.4+0.8=2.0, \\
& v_{1}-d_{\widehat{G}_{1}\left[\widehat{G}_{2}\right]_{\max }}\left(v_{2}, w_{1}\right)=0.6+0.6+0.6+0.8=2.6, \\
& v_{2}-d_{\widehat{G}_{1}\left[\widehat{G}_{2}\right]_{\max }}\left(v_{3}, w_{3}\right)=0.4+0.4+0.4+0.8=2.0, \\
& v_{2}-d_{\widehat{G}_{1}}\left[\widehat{G}_{2}\right]_{\max }\left(v_{3}, w_{2}\right)=0.4+0.4+0.4+0.8=2.0, \\
& v_{1}-d_{\widehat{G}_{1}\left[\widehat{G}_{2}\right]_{\max }}\left(v_{2}, w_{2}\right)=0.6+0.6+0.6+0.8=2.6 \text {, } \\
& v_{2}-d_{\widehat{G}_{1}\left[\widehat{G}_{2}\right]_{\max }}\left(v_{4}, w_{2}\right)=0.4+0.4+0.4+0.8=2.0 \text {, }
\end{aligned}
$$

$$
\begin{aligned}
& v_{1}-d_{\widehat{G}_{1}}\left[\widehat{G}_{2}\right]_{\max }\left(v_{1}, w_{2}\right)=0.6+0.6+0.6+0.8=2.6, \\
& v_{1}-d_{\widehat{G}_{1}}\left[\widehat{G}_{2}\right]_{\max }\left(v_{1}, w_{3}\right)=0.6+0.6+0.6=1.8, \\
& v_{1}-d_{\widehat{G}_{1}}\left[\widehat{G}_{2}\right]_{\max }\left(v_{2}, w_{3}\right)=0.6+0.6+0.6=1.8, \\
& v_{2}-d_{\widehat{G}_{1}\left[\widehat{G}_{2}\right]_{\max }}\left(v_{3}, w_{1}\right)=0.4+0.4+0.4=1.2, \\
& v_{2}-d_{\widehat{G}_{1}\left[\widehat{G}_{2}\right]_{\max }}\left(v_{4}, w_{1}\right)=0.4+0.4+0.4=1.2 .
\end{aligned}
$$

This example explains that the degrees of both methods are the same.

Theorem 7. If $\widehat{G}_{1}$ and are two FGSs such that $\nu_{i}^{\prime \prime} \leq \alpha_{1}$, then $v_{i}$-the degree of the vertex in the lexicographic-max product $\widehat{G}_{1}\left[\widehat{G}_{2}\right]_{\max }$ of FGSs $\widehat{G}_{1}$ and $\widehat{G}_{2}$ is given by $v_{i}-d_{\widehat{G}_{1}\left[\widehat{G}_{2}\right]_{\max }}\left(v_{i}, w_{j}\right)=d_{\widehat{G}_{2}^{*}}\left(w_{j}\right) \alpha_{1}\left(v_{i}\right)+\left[v_{i}^{\prime}-d_{\widehat{G}_{1}}\left(v_{i}\right)\right]\left|V_{2}\right|$.

Proof. Let $\widehat{G}_{1}=\left(\alpha_{1}, v_{1}^{\prime}, v_{2}^{\prime}, \ldots, v_{n}^{\prime}\right)$ and $\widehat{G}_{2}=\left(\alpha_{2}, v_{1}^{\prime \prime}, v_{2}^{\prime \prime}\right.$, $\left.\ldots, v_{n}^{\prime \prime}\right)$ be two FGSs such that $v_{i}^{\prime \prime} \leq \alpha_{1}$, then $v_{i}^{\prime \prime} \vee \alpha_{1}=\alpha_{1}$, $i=1,2, \ldots, n \cdot v_{i}$-the degree of the vertex in $\widehat{G}_{1}\left[\widehat{G}_{2}\right]_{\max }$ (lexicographic-max product) is defined as

$$
\begin{aligned}
v_{i}-d_{\widehat{G}_{1}\left[\widehat{G}_{2}\right]_{\max }}\left(v_{i}, w_{j}\right)= & \sum_{w_{j} w_{l} \in \hat{R}_{i}, v_{i}=v_{k}}\left(v_{i}^{\prime \prime}\left(w_{j} w_{l}\right) \vee \alpha_{1}\left(v_{i}\right)\right) \\
& +\sum_{v_{i}, v_{k} \in R_{i}^{\prime} w_{l} \in V_{2}} v_{i}^{\prime}\left(v_{i} v_{k}\right) \\
= & \sum_{w_{j} w_{l} \in \hat{R}_{i}, v_{i}=v_{k}} \alpha_{1}\left(v_{i}\right)+\left|V_{2}\right|\left[v_{i}^{\prime}-d_{\widehat{G}_{1}}\left(v_{i}\right)\right] \\
= & d_{\widehat{G}_{2}^{*}}\left(w_{j}\right) \alpha_{1}\left(v_{i}\right)+\left|V_{2}\right|\left[v_{i}^{\prime}-d_{\widehat{G}_{1}}\left(v_{i}\right)\right] .
\end{aligned}
$$

Corollary 1. If $\widehat{G}_{1}$ and $\widehat{G}_{2}$ are two FGSs such that $\nu_{i}^{\prime \prime} \leq \alpha_{1}$ and $\alpha_{1}$ is considered as a constant function having a value $c$, then $v_{i}$-the degree of the vertex in the lexicographic-max product $\widehat{G}_{1}\left[\widehat{G}_{2}\right]_{\max }$ of FGSs $\widehat{G}_{1}$ and $\widehat{G}_{2}$ is given by $v_{i}-d_{\widehat{G}_{1}} \widehat{G}_{1}\left[\widehat{G}_{2}\right]_{\max }\left(v_{i}\right.$, $\left.w_{j}\right)=d_{\widehat{G}_{2}^{*}}\left(w_{j}\right) c+\left[v_{i}-d_{\widehat{G}_{1}}\left(v_{i}\right)\right]\left|V_{2}\right|$.

Remark 1. If $\widehat{G}_{1}$ and $\widehat{G}_{2}$ are regular FGSs, then their lexicographic-max product $\widehat{G}_{1}\left[\widehat{G}_{2}\right]_{\max }$ is not a regular FGS.

Example 5. Consider two FGSs $\widehat{G}_{1}=\left(\alpha_{1}, v_{1}^{\prime}, v_{2}^{\prime}\right)$ and $G_{2}=$ $\left(\alpha_{2}, v_{1}^{\prime \prime}, v_{2}^{\prime \prime}\right)$ as shown in Figure 9.

It is clear from Figure 9 that each vertex of $\widehat{G}_{1}=\left(\alpha_{1}, v_{1}^{\prime}, v_{2}^{\prime}\right)$ has one $v_{1}^{\prime}$ edge with the same degree of membership, that is, 0.7. Hence, $\widehat{G}_{1}$ is a $0.7 v_{1}^{\prime}$-regular FGS. Moreover, each vertex of $\widehat{G}_{2}=\left(\alpha_{2}, v_{1}^{\prime \prime}, v_{2}^{\prime \prime}\right)$ has one $v_{1}^{\prime \prime}$ edge with the same degree of membership, that is, 0.5 . Hence, $G_{2}$ is a $0.5 \nu_{1}^{\prime \prime}$-regular FGS. The lexicographic-max product of $\widehat{G}_{1}=\left(\alpha_{1}, v_{1}^{\prime}, v_{2}^{\prime}\right)$ and $\widehat{G}_{2}=\left(\alpha_{2}, v_{1}^{\prime \prime}, v_{2}^{\prime \prime}\right)$ is shown in Figure 10.

In Figure 10, all green-colored edges are $v_{1}^{\prime}$ edges with a membership value 0.7 , all yellow-edges are $v_{2}^{\prime}$ edges with a 


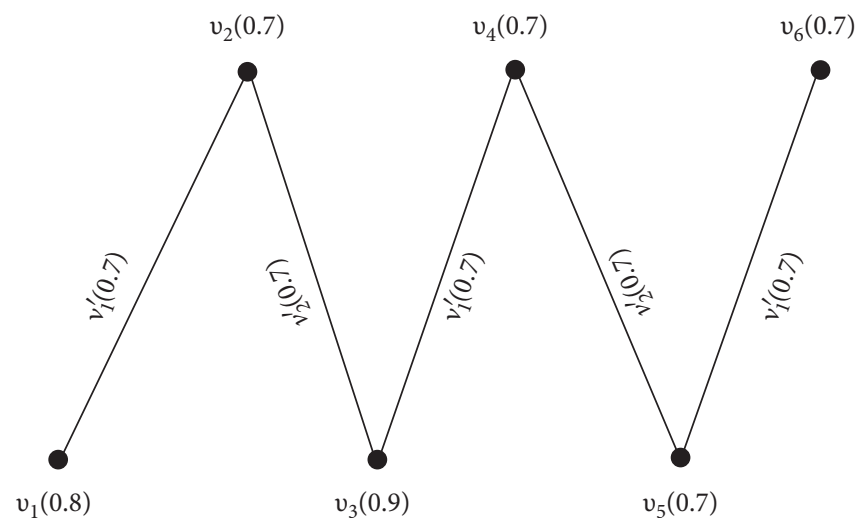

(a)

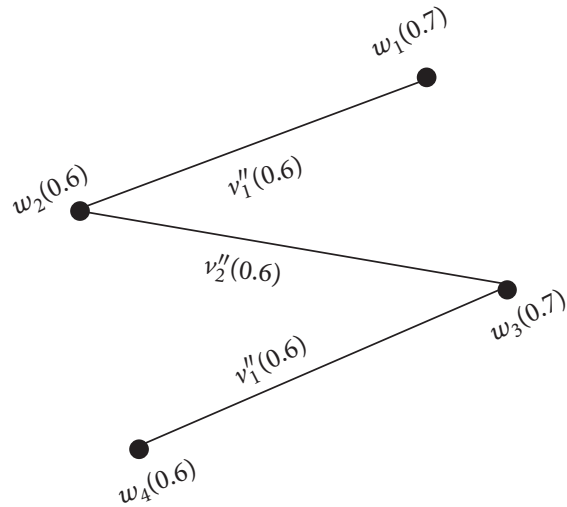

(b)

Figure 9: $v_{1}^{\prime}$-regular and $v_{1}^{\prime \prime}$-regular FGSs. (a) $\widehat{G}_{1}=\left(\alpha_{1}, v_{1}^{\prime}, v_{2}^{\prime}\right)$. (b) $\widehat{G}_{2}=\left(\alpha_{2}, v_{1}^{\prime \prime}, v_{2}^{\prime \prime}\right)$.

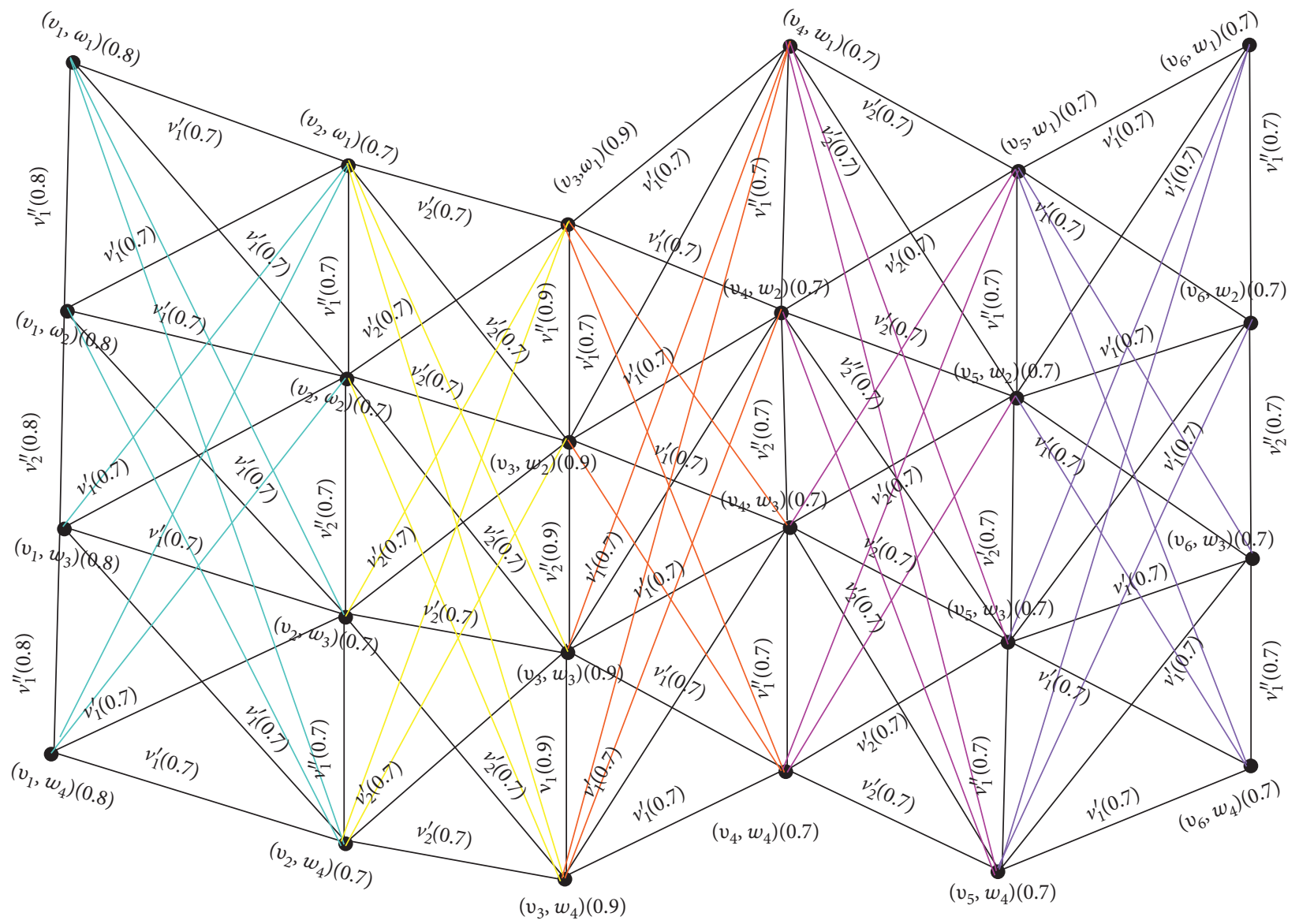

FIGURE 10: Lexicographic max-product of $\widehat{G}_{1}$ and $\widehat{G}_{2}$.

membership value 0.7 , all orange-colored edges are $v_{1}^{\prime}$ edges with a membership value 0.7 , all purple-colored edges are $v_{2}^{\prime}$ edges with a membership value 0.7 , and all blue-colored edges are $v_{1}^{\prime}$ edges with a membership value 0.7 . It is clear from Figure 10 that each vertex in the lexicographic-max product $\widehat{G}_{1}\left[\widehat{G}_{2}\right]_{\max }$ does not have any type of edges with the same membership value. For example, the vertex $\left(v_{1}, w_{1}\right)$ has five $v_{1}$ edges, four $v_{1}$ edges with a 0.7 membership value, and one $v_{1}$ edge with a membership value 0.8 , while the vertex $\left(v_{3}, w_{1}\right)$ has five $v_{1}$ edges, four $v_{1}$ edges having a membership value 0.7 , and one $\nu_{1}$ edge with a membership value 0.9 . Hence, $\widehat{G}_{1}\left[\widehat{G}_{2}\right]_{\max }$ is not a regular FGS. This example shows that the lexicographic-max product of two regular FGSs is not a regular FGS. 
Remark 2. If $\widehat{G}_{1}$ and $\widehat{G}_{2}$ are partially regular FGSs, then the lexicographic-max product $\widehat{G}_{1}\left[\widehat{G}_{2}\right]_{\max }$ is a partially regular FGS since $\widehat{G}_{1}^{*}$ and $G_{2}^{*}$ are regular FGSs.

Theorem 8. If $\widehat{G}_{1}$ and $\widehat{G}_{2}$ are two FGSs such that $\nu_{i}^{\prime \prime} \geq \alpha_{1}$, then the lexicographic-max product $\widehat{G}_{1}\left[\widehat{G}_{2}\right]_{\max }$ is a $v_{i}$-regular $F G S$ if and only if $\widehat{G}_{1}$ is a $v_{i}^{\prime}$-regular FGS and $\widehat{G}_{2}$ is a $\nu_{i}^{\prime \prime}$-regular FGS.

Proof. Let $\widehat{G}_{1}=\left(\alpha_{1}, v_{1}^{\prime}, v_{2}^{\prime}, \ldots, v_{n}^{\prime}\right)$ and $\widehat{G}_{2}=\left(\alpha_{2}, v_{1}^{\prime \prime}, v_{2}^{\prime \prime}\right.$, $\left.\ldots, v_{n}^{\prime \prime}\right)$ be two FGSs such that $\nu_{i}^{\prime \prime} \geq \alpha_{1}$. Then, $v_{i}$-the degree of the vertex in the lexicographic-max product $\widehat{G}_{1}\left[\widehat{G}_{2}\right]_{\max }$ is given by

$$
v_{i}-d_{\widehat{G}_{1}\left[\widehat{G}_{2}\right]_{\max }}\left(v_{i}, w_{j}\right)=\left[v_{i}^{\prime \prime}-d_{\widehat{G}_{2}}\left(w_{j}\right)\right]+\left[v_{i}^{\prime}-d_{\widehat{G}_{1}}\left(v_{i}\right)\right]\left|V_{2}\right| .
$$

Assume that $\widehat{G}_{1}=\left(\alpha_{1}, v_{1}^{\prime}, v_{2}^{\prime}, \ldots, v_{n}^{\prime}\right)$ is a $v_{i}^{\prime}$-regular FGS of degree $k_{1}$ and $\widehat{G}_{2}=\left(\alpha_{2}, v_{1}^{\prime \prime}, v_{2}^{\prime \prime}, \ldots, v_{n}^{\prime \prime}\right)$ is a $\nu_{i}^{\prime \prime}$-regular FGS of degree $k_{2}$, then

$$
v_{i}-d_{\widehat{G}_{1}\left[\widehat{G}_{2}\right]_{\max }}\left(v_{i}, w_{j}\right)=k_{2}+k_{1}\left|V_{2}\right| .
$$

Since $k_{1}, k_{2}$, and $\left|V_{2}\right|$ are constants, so $v_{i}-d_{\widehat{G}_{1}\left[\widehat{G}_{2}\right]_{\max }}$ is a constant for all elements of $V_{1} \times V_{2}$. Thus, the lexicographicmax product $\widehat{G}_{1}\left[\widehat{G}_{2}\right]_{\max }$ is a $\nu_{i}$-regular FGS.

Conversely, suppose that $\widehat{G}_{1}=\left(\alpha_{1}, v_{1}^{\prime}, v_{2}^{\prime}, \ldots, v_{n}^{\prime}\right)$ and $\widehat{G}_{2}=\left(\alpha_{2}, v_{1}^{\prime \prime}, v_{2}^{\prime \prime}, \ldots, v_{n}^{\prime \prime}\right)$ are two FGSs such that $\nu_{i}^{\prime \prime} \geq \alpha_{1}$ and the lexicographic-max product $\widehat{G}_{1}\left[\widehat{G}_{2}\right]_{\max }=\left(\alpha, \nu_{1}, \nu_{2}, \ldots\right.$, $\left.v_{n}\right)$ is a $v_{i}$-regular FGS. Then, $v_{i}$-the degrees of vertices $\left(v_{1}, v_{2}\right)$ and $\left(w_{1}, w_{2}\right)$ in $V_{1} \times V_{2}$ are equal, that is,

$$
\begin{aligned}
v_{i}-d_{\widehat{G}_{1}\left[\widehat{G}_{2}\right]_{\max }\left(v_{1}, v_{2}\right)=} v_{i}-d_{\widehat{G}_{1}}\left[\widehat{G}_{2}\right]_{\max }\left(w_{1}, w_{2}\right), \\
{\left[v_{i}^{\prime \prime}-d_{\widehat{G}_{2}}\left(v_{2}\right)\right]+\left[v_{i}^{\prime}-d_{\widehat{G}_{1}}\left(v_{1}\right)\right]\left|V_{2}\right|=\left[v_{i}^{\prime \prime}-d_{\widehat{G}_{2}}\left(w_{2}\right)\right] } \\
+\left[v_{i}^{\prime}-d_{\widehat{G}_{1}}\left(w_{1}\right)\right]\left|V_{2}\right| .
\end{aligned}
$$

Fixing $v \in V_{2}$ and considering $\left(v_{1}, v\right)$ and $\left(w_{1}, v\right)$ in $V_{1} \times V_{2}$, where $v_{1}$ and $w_{1}$ are taken as the arbitrary objects of $V_{1}$, then the above expression is converted as

$$
\begin{gathered}
{\left[v_{i}^{\prime \prime}-d_{\widehat{G}_{2}}(v)\right]+\left[v_{i}^{\prime}-d_{\widehat{G}_{1}}\left(v_{1}\right)\right]\left|V_{2}\right|=\left[v_{i}^{\prime \prime}-d_{\widehat{G}_{2}}(v)\right]+\left[v_{i}^{\prime}-d_{\widehat{G}_{1}}\left(w_{1}\right)\right]\left|V_{2}\right|,} \\
{\left[v_{i}^{\prime}-d_{\widehat{G}_{1}}\left(v_{1}\right)\right]\left|V_{2}\right|=\left[v_{i}^{\prime}-d_{\widehat{G}_{1}}\left(w_{1}\right)\right]\left|V_{2}\right|,} \\
v_{i}-d_{\widehat{G}_{1}}\left(v_{1}\right)=v_{i}-d_{\widehat{G}_{1}}\left(w_{1}\right) .
\end{gathered}
$$

This holds for all vertices $v_{1}$ and $w_{1}$ of $V_{1}$. Thus, $\widehat{G}_{1}=$ $\left(\alpha_{1}, v_{1}^{\prime}, v_{2}^{\prime}, \ldots, v_{n}^{\prime}\right)$ is a $v_{i}^{\prime}$-regular FGS.
Now fixing $v^{\prime} \in V_{1}$ and considering $\left(v^{\prime}, v_{2}\right)$ and $\left(v^{\prime}, w_{2}\right)$ in $V_{1} \times V_{2}$, where $v_{2}$ and $w_{2}$ are arbitrary elements of the vertex set $V_{1}$, then the above given equation becomes

$$
\begin{gathered}
{\left[v_{i}^{\prime \prime}-d_{\widehat{G}_{2}}\left(v_{2}\right)\right]+\left[v_{i}^{\prime}-d_{\widehat{G}_{1}}\left(v^{\prime}\right)\right]\left|V_{2}\right|=\left[v_{i}^{\prime \prime}-d_{\widehat{G}_{2}}\left(w_{2}\right)\right]+\left[v_{i}^{\prime}-d_{\widehat{G}_{1}}\left(v^{\prime}\right)\right]\left|V_{2}\right|} \\
{\left[v_{i}^{\prime \prime}-d_{\widehat{G}_{2}}\left(v_{2}\right)\right]=\left[v_{i}^{\prime \prime}-d_{\widehat{G}_{2}}\left(w_{2}\right)\right] .}
\end{gathered}
$$

This holds for all elements $v_{2}$ and $w_{2}$ of $V_{2}$. Thus, $\widehat{G}_{2}=$ $\left(\alpha_{2}, v_{1}^{\prime \prime}, v_{2}^{\prime \prime}, \ldots, v_{n}^{\prime \prime}\right)$ is a $v_{i}^{\prime \prime}$-regular FGS.

Theorem 9. If $\widehat{G}_{1}$ is a $v_{i}^{\prime}$-regular FGS and $\widehat{G}_{2}$ is a partially regular FGS such that $\nu_{i}^{\prime \prime} \vee \alpha_{1}$ is a constant, then the lexicographic-max product $\widehat{G}_{1}\left[\widehat{G}_{2}\right]_{\max }$ of $\widehat{G}_{1}$ and $\widehat{G}_{2}$ is a $v_{i}$-regular FGS.

Proof. Let $\widehat{G}_{1}=\left(\alpha_{1}, v_{1}^{\prime}, v_{2}^{\prime}, \ldots, v_{n}^{\prime}\right)$ be a $v_{i}^{\prime}-$ regular FGS of degree $k_{1}$ and $\widehat{G}_{2}=\left(\alpha_{2}, \nu_{1}^{\prime \prime}, \nu_{2}^{\prime \prime}, \ldots, \nu_{n}^{\prime \prime}\right)$ be a partially regular FGS such that $\widehat{G}_{2}^{*}=\left(V_{2}, R_{1}^{\prime \prime}, R_{2}^{\prime \prime}, \ldots, R_{n}^{\prime \prime}\right)$ (underlying GS) is an $r_{1}$-regular GS.
Moreover, $\nu_{i}^{\prime \prime} \vee \alpha_{1}$ is a constant, that is, $\nu_{i}^{\prime \prime} \vee \alpha_{1}=c$, where $c$ is a constant.

Then, the degree of the vertex in the lexicographic-max product $\widehat{G}_{1}\left[\widehat{G}_{2}\right]_{\max }$ of FGSs $\widehat{G}_{1}$ and $\widehat{G}_{2}$ is given by

$$
\begin{aligned}
v_{i}-d_{\widehat{G}_{1}\left[\widehat{G}_{2}\right]_{\max }}\left(u_{i}, w_{j}\right) & =d_{\widehat{G}_{2}^{*}}\left(w_{j}\right)\left(\ddot{v}_{i} \vee \alpha_{1}\right)+\left[v_{i}^{\prime}-d_{\widehat{G}_{1}}\left(v_{i}\right)\right]\left|V_{2}\right| \\
& =d_{\widehat{G}_{2}^{*}}\left(w_{j}\right) c+\left[v_{i}^{\prime}-d_{\widehat{G}_{1}}\left(v_{i}\right)\right]\left|V_{2}\right| \\
& =r_{1} c+k_{1}\left|V_{2}\right| .
\end{aligned}
$$

Since $c, k_{1}, r_{1}$, and $\left|V_{2}\right|$ are constants, so $v_{i}-d_{\widehat{G}_{1}\left[\widehat{G}_{1}\right]}$ is a constant for all elements of $V_{1} \times V_{2}$. Thus, the lexicographic- 
max product $\widehat{G}_{1}\left[\widehat{G}_{2}\right]_{\max }$ of FGSs $\widehat{G}_{1}$ and $\widehat{G}_{2}$ is a $\nu_{i}$-regular FGS.

Theorem 10. If $\widehat{G}_{1}$ and $\widehat{G}_{2}$ are two FGSs such that $\nu_{i}^{\prime} \geq \alpha_{2}$, then the lexicographic-max product $\widehat{G}_{1}\left[\widehat{G}_{2}\right]_{\max }$ is a $v_{i}$-regular FGS if and only if $\widehat{G}_{1}$ is a - $v_{i}^{\prime}$-regular FGS and $\widehat{G}_{2}$ is a $\nu_{i}^{\prime \prime}$-regular FGS.

Proof. Let $\widehat{G}_{1}=\left(\alpha_{1}, v_{1}^{\prime}, v_{2}^{\prime}, \ldots, v_{n}^{\prime}\right)$ and $G_{2}=\left(\alpha_{2}, v_{1}^{\prime \prime}, v_{2}^{\prime \prime}, \ldots\right.$, $\left.v_{n}^{\prime \prime}\right)$ be two FGSs such that $v_{i}^{\prime} \geq \alpha_{2}$. Then, $v_{i}$-the degree of the vertex in the lexicographic-max product $\widehat{G}_{1}\left[\widehat{G}_{2}\right]_{\max }$ is given by

$$
v_{i}-d_{\widehat{G}_{1}}\left[\widehat{G}_{2}\right]_{\max }\left(v_{i}, w_{j}\right)=\left|V_{1}\right|\left[v_{i}^{\prime \prime}-d_{\widehat{G}_{2}}\left(w_{j}\right)\right]+\left[v_{i}^{\prime}-d_{\widehat{G}_{1}}\left(v_{i}\right)\right] .
$$

Assume that $\widehat{G}_{1}=\left(\alpha_{1}, v_{1}^{\prime}, v_{2}^{\prime}, \ldots, v_{n}^{\prime}\right)$ is a $v_{i}^{\prime}$-regular FGS of degree $k_{1}$ and $\widehat{G}_{2}=\left(\alpha_{2}, v_{1}^{\prime \prime}, v_{2}^{\prime \prime}, \ldots, v_{n}^{\prime \prime}\right)$ is a $v_{i}^{\prime \prime}$-regular FGS of degree $k_{2}$ :

$$
v_{i}-d_{\widehat{G}_{1}\left[\widehat{G}_{2}\right]_{\max }}\left(v_{i}, w_{j}\right)=\left|V_{1}\right| k_{2}+k_{1} .
$$

Since $k_{1}, k_{2}$, and $\left|V_{1}\right|$ are constants, so $v_{i}-d_{\widehat{G}_{2}\left[\widehat{G}_{1}\right]_{\max }}$ is constant for all elements of $V_{1} \times V_{2}$. Thus, the lexicographicmax product $\widehat{G}_{2}\left[\widehat{G}_{1}\right]_{\max }=\left(\alpha, v_{1}, v_{2}, \ldots, v_{n}\right)$ is a $v_{i}$-regular FGS.

Conversely, suppose that the lexicographic-max product $\widehat{G}_{2}\left[\widehat{G}_{1}\right]_{\max }=\left(\alpha, v_{1}, v_{2}, \ldots, v_{n}\right)$ is a $v_{i}-$ regular FGS. Then, $v_{i}$-the degrees of two vertices $\left(v_{1}, v_{2}\right)$ and $\left(w_{1}, w_{2}\right)$ in $V_{1} \times$ $V_{2}$ are equal, that is,

$$
\begin{aligned}
v_{i}-d_{\widehat{G}_{2}}\left[\widehat{G}_{1}\right]_{\max }\left(v_{1}, v_{2}\right)= & v_{i}-d_{\widehat{G}_{2}\left[\widehat{G}_{1}\right]_{\max }}\left(w_{1}, w_{2}\right), \\
\left|V_{1}\right|\left[v_{i}^{\prime \prime}-d_{\widehat{G}_{2}}\left(v_{2}\right)\right]+\left[v_{i}^{\prime}-d_{\widehat{G}_{1}}\left(v_{1}\right)\right]= & \left|V_{1}\right|\left[v_{i}^{\prime \prime}-d_{\widehat{G}_{2}}\left(w_{2}\right)\right] \\
& +\left[v_{i}^{\prime}-d_{\widehat{G}_{1}}\left(w_{1}\right)\right] .
\end{aligned}
$$

Fixing $v \in V_{2}$ and considering $\left(v_{1}, v\right)$ and $\left(w_{1}, v\right)$ in $V_{1} \times V_{2}$, where $v_{1}$ and $w_{1}$ are arbitrary elements of the vertex set $V_{1}$, then the above equation becomes

$$
\begin{aligned}
\left|V_{1}\right|\left[v_{i}^{\prime \prime}-d_{\widehat{G}_{2}}(v)\right]+\left[v_{i}^{\prime}-d_{\widehat{G}_{1}}\left(v_{1}\right)\right]= & \left|V_{1}\right|\left[\nu_{i}^{\prime \prime}-d_{\widehat{G}_{2}}(v)\right] \\
& +\left[v_{i}^{\prime}-d_{\widehat{G}_{1}}\left(w_{1}\right)\right], \\
v_{i}^{\prime}-d_{\widehat{G}_{1}}\left(v_{1}\right)= & v_{i}^{\prime}-d_{\widehat{G}_{1}}\left(w_{1}\right) .
\end{aligned}
$$

This holds for all elements $v_{1}$ and $w_{1}$ of $V_{1}$. Thus, $\widehat{G}_{1}=$ $\left(\alpha_{1}, v_{1}^{\prime}, v_{2}^{\prime}, \ldots, v_{n}^{\prime}\right)$ is a $v_{i}^{\prime}$-regular FGS.
Now fixing $v \prime \in V_{1}$ and considering $\left(v^{\prime}, v_{2}\right)$ and $\left(v \prime, w_{2}\right)$ in $V_{1} \times V_{2}$, where $v_{2}$ and $w_{2}$ are arbitrary elements of the vertex set $V_{2}$, then the above equation becomes

$$
\begin{aligned}
&\left|V_{1}\right|\left[v_{i}^{\prime \prime}-d_{\widehat{G}_{2}}\left(v_{2}\right)\right]+\left[v_{i}^{\prime \prime}-d_{\widehat{G}_{1}}(v \prime)\right]=\left|V_{1}\right|\left[v_{i}^{\prime \prime}-d_{\widehat{G}_{2}}\left(w_{2}\right)\right] \\
&+\left[v_{i}^{\prime \prime}-d_{\widehat{G}_{1}}(v \prime)\right], \\
&\left|V_{1}\right|\left[v_{i}^{\prime \prime}-d_{\widehat{G}_{2}}\left(v_{2}\right)\right]=\left|V_{1}\right|\left[v_{i}^{\prime \prime}-d_{\widehat{G}_{2}}\left(w_{2}\right)\right], \\
& {\left[v_{i}^{\prime \prime}-d_{\widehat{G}_{2}}\left(v_{2}\right)\right]=\left[v_{i}^{\prime \prime}-d_{\widehat{G}_{2}}\left(w_{2}\right)\right] . }
\end{aligned}
$$

This holds for all vertices $v_{2}$ and $w_{2}$ of $V_{2}$. Thus, $\widehat{G}_{2}=$ $\left(\alpha_{2}, v_{1}^{\prime \prime}, v_{2}^{\prime \prime}, \ldots, v_{n}^{\prime \prime}\right)$ is a $v_{i}^{\prime \prime}$-regular FGS.

Definition 5. The total degree of the 5 lexicographic-max product $\widehat{G}_{1}\left[\widehat{G}_{2}\right]_{\max }=\left(\alpha, v_{1}, v_{2}, \ldots, v_{n}\right)$ of FGSs $\widehat{G}_{1}=\left(\alpha_{1}, v_{1}^{\prime}, v_{2}^{\prime}, \ldots, v_{n}^{\prime}\right)$ and $\widehat{G}_{2}=\left(\alpha_{2}, v_{1}^{\prime \prime}, v_{2}^{\prime \prime}, \ldots, v_{n}^{\prime \prime}\right)$ is defined as

$$
\begin{aligned}
t d_{\widehat{G}_{1}\left[\widehat{G}_{2}\right]_{\max }}\left(u_{i}, v_{j}\right)= & \sum_{u_{i} u_{k} \in R_{p}^{\prime} v_{l} \in V_{2}} v_{i}^{\prime}\left(u_{i} u_{k}\right) \\
& +\sum_{v_{j} v_{l} \in R_{i}^{\prime \prime} u_{i}=u_{k}}\left[v_{j}^{\prime \prime}\left(v_{j} v_{l}\right) \vee \alpha_{1}\left(u_{i}\right)\right]+\alpha\left(u_{i}, v_{j}\right) .
\end{aligned}
$$
as

$v_{i}$-the total degree of the vertex in $\widehat{G}_{1}\left[\widehat{G}_{2}\right]_{\max }$ is defined

$$
\begin{aligned}
v_{i}-t{\widehat{G_{1}}}_{\left.\widehat{G}_{2}\right]_{\max }}\left(u_{i}, v_{j}\right)= & \sum_{u_{i} u_{k} \in R_{i}^{\prime}, v_{l} \in V_{2}} v_{i}^{\prime}\left(u_{i} u_{k}\right) \\
& +\sum_{v_{j} v_{l} \in R_{i}^{\prime \prime}, u_{i}=u_{k}}\left[v_{i}^{\prime \prime}\left(v_{j} v_{l}\right) \vee \alpha_{1}\left(u_{i}\right)\right] \\
& +\frac{\alpha\left(u_{i}, v_{j}\right)}{n} .
\end{aligned}
$$

Example 6. Consider two FGSs $\widehat{G}_{1}=\left(\alpha_{1}, v_{1}^{\prime}, v_{2}^{\prime}\right)$ and $\widehat{G}_{2}=\left(\alpha_{2}, v_{1}^{\prime \prime}, v_{2}^{\prime \prime}\right)$, as shown in Figure 11 .

The lexicographic-max product of $\widehat{G}_{1}=\left(\alpha_{1}, v_{1}^{\prime}, v_{2}^{\prime}\right)$ and $\widehat{G}_{2}=\left(\alpha_{2}, v_{1}^{\prime \prime}, v_{2}^{\prime \prime}\right)$ is shown in Figure 12.

The total degrees of vertices in the lexicographic-max product are computed as 


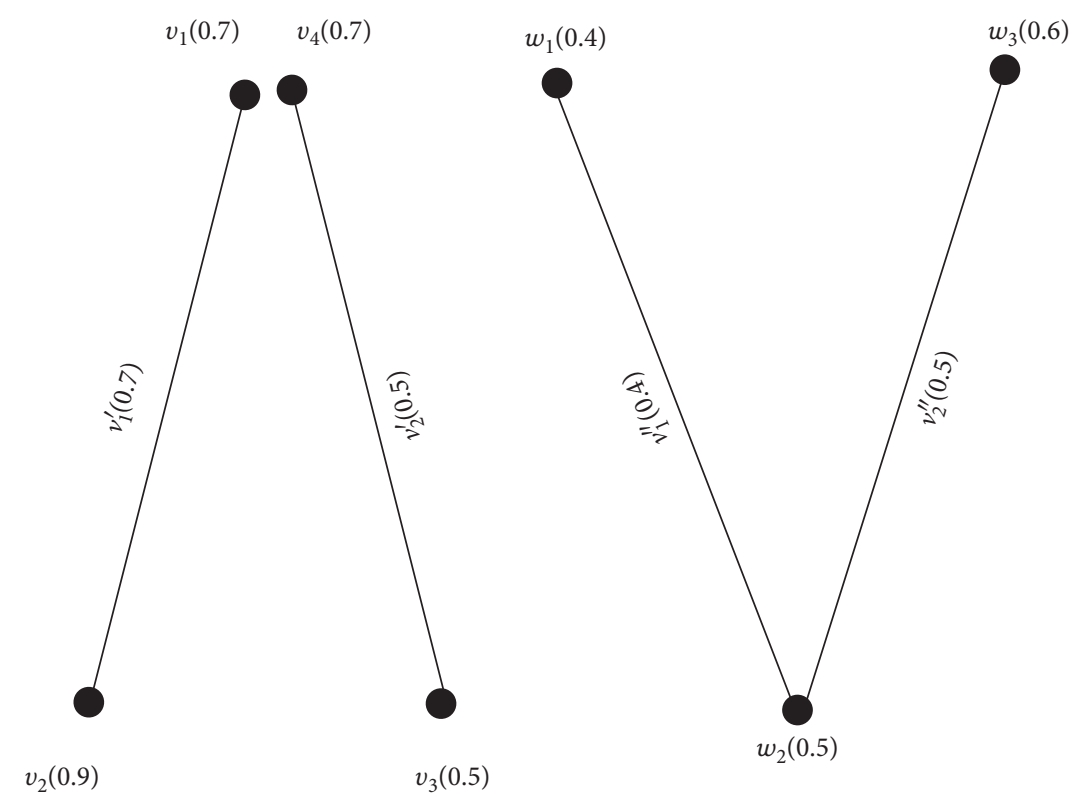

(a)

(b)

Figure 11: Two FGSs. (a) $G_{1}=\left(\alpha_{1}, v_{1}^{\prime}, v_{2}^{\prime}\right)$. (b) $G_{2}=\left(\alpha_{2}, v_{1}^{\prime \prime}, v_{2}^{\prime \prime}\right)$.

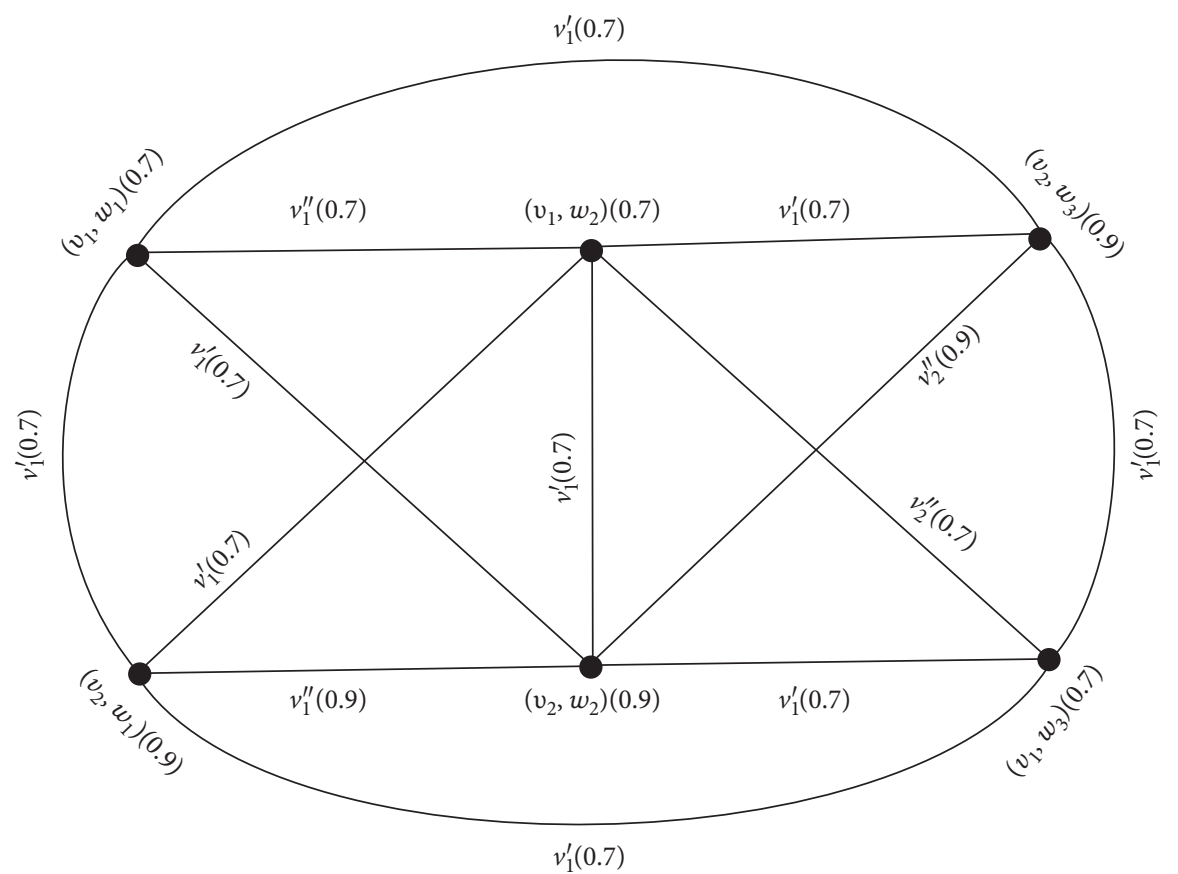

(a)

Figure 12: Continued. 


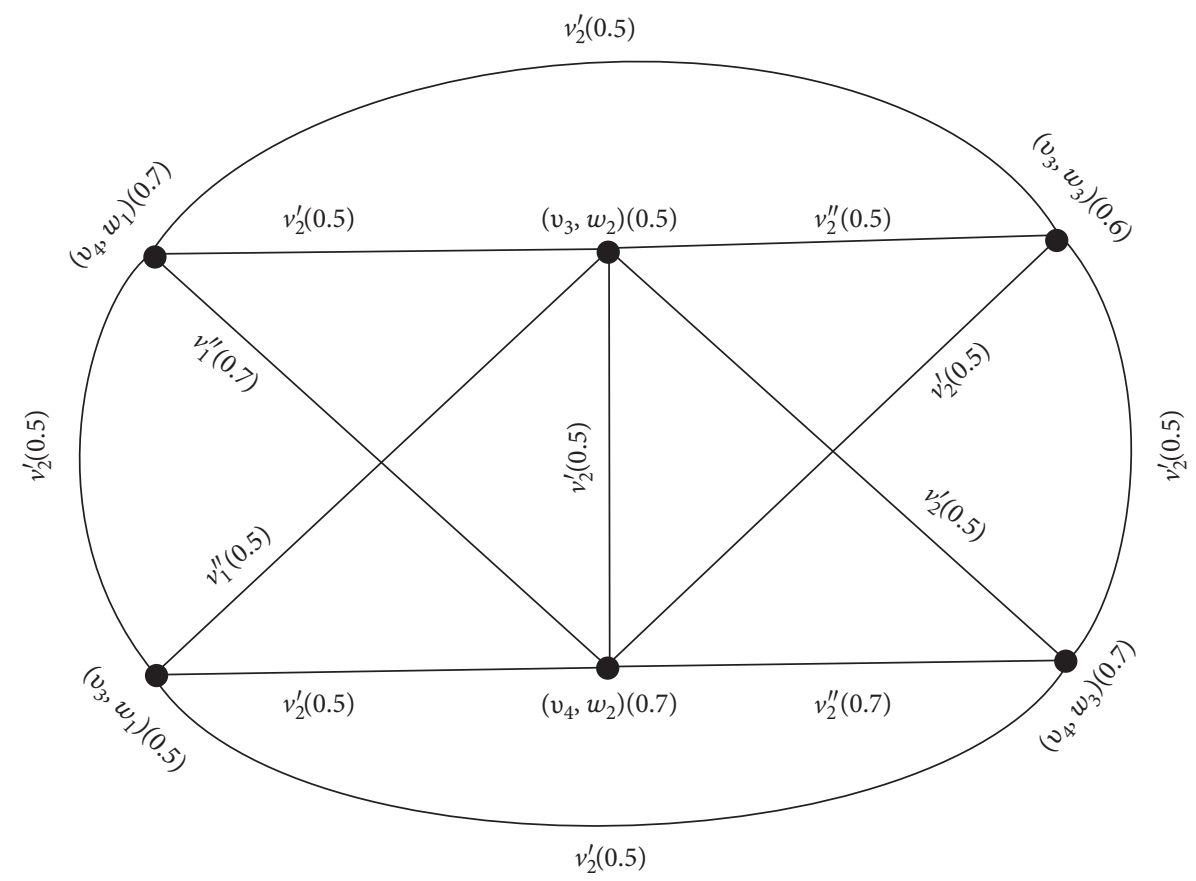

(b)

FIGURE 12: Lexicographic-max product $\widehat{G}_{1}\left[\widehat{G}_{2}\right]_{\max }$.

$$
\begin{aligned}
t d_{\widehat{G}_{1}\left[\widehat{G}_{2}\right]_{\max }}\left(v_{i}, w_{j}\right)= & \sum_{v_{i} v_{k} \in R_{\dot{p}}^{\prime} w_{l} \in V_{2}} v_{i}^{\prime}\left(v_{i} v_{k}\right) \\
& +\sum_{\substack{w_{j} w_{l} \in R_{j}^{\prime \prime}, v_{i}=v_{k}\\
}}\left[v_{j}^{\prime \prime}\left(w_{j} w_{l}\right) \vee \alpha_{1}\left(v_{i}\right)\right] \\
& \alpha\left(v_{i}, w_{j}\right),
\end{aligned}
$$

$$
\begin{aligned}
& t d_{\widehat{G}_{1}}\left[\widehat{G}_{2}\right]_{\max }\left(v_{1}, w_{1}\right)=v_{1}^{\prime}\left(v_{1} v_{2}\right)+v_{1}^{\prime}\left(v_{1} v_{2}\right) \\
& +v_{1}^{\prime}\left(v_{1} v_{2}\right)+\left(v_{1}^{\prime \prime}\left(w_{1} w_{2}\right) \vee \alpha_{1}\left(v_{1}\right)\right) \\
& +\alpha\left(v_{1}, w_{1}\right) \\
& =0.7+0.7+0.7+(0.4 \vee 0.7)+0.7 \\
& =2.1+0.7+0.7 \\
& =3.5 \text {, }
\end{aligned}
$$

$$
\begin{aligned}
& t d_{\widehat{G}_{1}}\left[\widehat{G}_{2}\right]_{\max }\left(v_{2}, w_{1}\right)=v_{1}^{\prime}\left(v_{2} v_{1}\right)+v_{1}^{\prime}\left(v_{2} v_{1}\right) \\
& +v_{1}^{\prime}\left(v_{2} v_{1}\right)+\left(v_{1}^{\prime \prime}\left(w_{1} w_{2}\right) \vee \alpha_{2}\left(v_{2}\right)\right) \\
& +\alpha\left(v_{2}, w_{1}\right) \\
& =0.7+0.7+0.7+(0.4 \vee 0.9)+0.9 \\
& =2.1+0.9+0.9 \\
& =3.9 \text {, }
\end{aligned}
$$

$$
\begin{aligned}
t d_{\widehat{G}_{1}\left[\widehat{G}_{2}\right]_{\max }\left(v_{2}, w_{3}\right)=} & v_{1}^{\prime}\left(v_{2} v_{1}\right)+v_{1}^{\prime}\left(v_{2} v_{1}\right)+v_{1}^{\prime}\left(v_{2} v_{1}\right) \\
& +\left(v_{2}^{\prime \prime}\left(w_{3} w_{2}\right) \vee \alpha_{1}\left(v_{2}\right)\right)+\alpha\left(v_{2}, w_{3}\right) \\
= & 0.7+0.7+0.7+(0.5 \vee 0.9)+0.9 \\
= & 2.1+0.9+0.9 \\
= & 3.9
\end{aligned}
$$

$$
\begin{aligned}
t d_{\widehat{G}_{1}\left[\widehat{G}_{2}\right]_{\max }\left(v_{4}, w_{1}\right)=} & v_{2}^{\prime}\left(v_{4} v_{3}\right)+v_{2}^{\prime}\left(v_{4} v_{3}\right)+v_{2}^{\prime}\left(v_{4} v_{3}\right) \\
& +\left(v_{1}^{\prime \prime}\left(w_{1} w_{2}\right) \vee \alpha_{1}\left(v_{4}\right)\right)+\alpha\left(v_{4}, w_{1}\right) \\
= & 0.5+0.5+0.5+(0.4 \vee 0.7)+0.7 \\
= & 1.5+0.7+0.7 \\
= & 2.9
\end{aligned}
$$




$$
\begin{aligned}
t d_{\widehat{G}_{1}\left[\widehat{G}_{2}\right]_{\max }}\left(v_{3}, w_{1}\right)= & v_{2}^{\prime}\left(v_{3} v_{4}\right)+v_{2}^{\prime}\left(v_{3} v_{4}\right)+v_{2}^{\prime}\left(v_{3} v_{4}\right) \\
& +\left(v_{1}^{\prime \prime}\left(w_{1} w_{2}\right) \vee \alpha_{1}\left(v_{3}\right)\right)+\alpha\left(v_{3}, w_{1}\right) \\
= & 0.5+0.5+0.5+(0.4 \vee 0.5)+0.5 \\
= & 1.5+0.5+0.5 \\
= & 2.5,
\end{aligned}
$$

$$
\begin{aligned}
t d_{\widehat{G}_{1}\left[\widehat{G}_{2}\right]_{\max }}\left(v_{4}, w_{3}\right)= & v_{2}^{\prime}\left(v_{4} v_{3}\right)+v_{2}^{\prime}\left(v_{4} v_{3}\right)+v_{2}^{\prime}\left(v_{4} v_{3}\right) \\
& +\left(v_{2}^{\prime \prime}\left(w_{3} w_{2}\right) \vee \alpha_{1}\left(v_{4}\right)\right)+\alpha\left(v_{4}, w_{3}\right) \\
= & 0.5+0.5+0.5+(0.5 \vee 0.7)+0.7 \\
= & 1.5+0.7+0.7 \\
= & 2.9,
\end{aligned}
$$

$$
\begin{aligned}
t d_{\widehat{G}_{1}\left[\widehat{G}_{2}\right]_{\max }}\left(v_{3}, w_{3}\right)= & v_{2}^{\prime}\left(v_{3} v_{4}\right)+v_{2}^{\prime}\left(v_{3} v_{4}\right)+v_{2}^{\prime}\left(v_{3} v_{4}\right) \\
& +\left(v_{2}^{\prime \prime}\left(w_{3} w_{2}\right) \vee \alpha_{1}\left(v_{3}\right)\right)+\alpha\left(v_{3}, w_{3}\right) \\
= & 0.5+0.5+0.5+(0.5 \vee 0.5)+0.6 \\
= & 1.5+0.5+0.6 \\
= & 2.6,
\end{aligned}
$$

$$
\begin{aligned}
t d_{\widehat{G}_{1}\left[\widehat{G}_{2}\right]_{\max }\left(v_{3}, w_{2}\right)=} & v_{2}^{\prime}\left(v_{3} v_{4}\right)+v_{2}^{\prime}\left(v_{3} v_{4}\right)+v_{2}^{\prime}\left(v_{3} v_{4}\right) \\
& +\left(v_{1}^{\prime \prime}\left(w_{2} w_{1}\right) \vee \alpha_{1}\left(v_{3}\right)\right) \\
& +\left(v_{2}^{\prime \prime}\left(w_{2} w_{3}\right) \vee \alpha_{1}\left(v_{3}\right)\right)+\alpha\left(v_{3}, w_{2}\right) \\
= & 0.5+0.5+0.5+(0.4 \vee 0.5) \\
& +(0.5 \vee 0.5)+0.5 \\
= & 1.5+0.5+0.5+0.5 \\
= & 3.0,
\end{aligned}
$$

$$
\begin{aligned}
t d_{\widehat{G}_{1}\left[\widehat{G}_{2}\right]_{\max }}\left(v_{4}, w_{2}\right)= & v_{2}^{\prime}\left(v_{4} v_{3}\right)+v_{2}^{\prime}\left(v_{4} v_{3}\right) \\
& +v_{2}^{\prime}\left(v_{4} v_{3}\right)+\left(v_{1}^{\prime \prime}\left(w_{2} w_{1}\right) \vee \alpha_{1}\left(v_{4}\right)\right) \\
& +\left(v_{2}^{\prime \prime}\left(w_{2} w_{3}\right) \vee \alpha_{1}\left(v_{4}\right)\right)+\alpha\left(v_{4}, w_{2}\right) \\
= & 0.5+0.5+0.5+(0.4 \vee 0.7) \\
& +(0.5 \vee 0.7)+0.7 \\
= & 1.5+0.7+0.7+0.7 \\
= & 3.6,
\end{aligned}
$$

$$
\begin{aligned}
t d_{\widehat{G}_{1}\left[\widehat{G}_{2}\right]_{\max }}\left(v_{1}, w_{2}\right)= & v_{1}^{\prime}\left(v_{1} v_{2}\right)+v_{1}^{\prime}\left(v_{1} v_{2}\right)+v_{1}^{\prime}\left(v_{1} v_{2}\right) \\
& +\left(v_{1}^{\prime \prime}\left(w_{2} w_{1}\right) \vee \alpha_{1}\left(v_{1}\right)\right) \\
& +\left(v_{2}^{\prime \prime}\left(w_{2} w_{3}\right) \vee \alpha_{1}\left(v_{1}\right)\right)+\alpha\left(v_{1}, w_{2}\right) \\
= & 0.7+0.7+0.7+(0.4 \vee 0.7) \\
& +(0.5 \vee 0.7)+0.7 \\
= & 2.1+0.7+0.7+0.7 \\
= & 4.2
\end{aligned}
$$

$$
\begin{aligned}
t d_{\widehat{G}_{1}\left[\widehat{G}_{2}\right]_{\max }}\left(v_{2}, w_{2}\right)= & v_{1}^{\prime}\left(v_{2} v_{1}\right)+v_{1}^{\prime}\left(v_{2} v_{1}\right)+v_{1}^{\prime}\left(v_{2} v_{1}\right) \\
& +\left(v_{1}^{\prime \prime}\left(w_{2} w_{1}\right) \vee \alpha_{1}\left(v_{2}\right)\right) \\
& +\left(v_{2}^{\prime \prime}\left(w_{2} w_{3}\right) \vee \alpha_{1}\left(v_{2}\right)\right)+\alpha\left(v_{2}, w_{2}\right) \\
= & 0.7+0.7+0.7+(0.4 \vee 0.9) \\
& +(0.5 \vee 0.9)+0.9 \\
= & 2.1+0.9+0.9+0.9 \\
= & 4.8 .
\end{aligned}
$$

To calculate $v_{i}$-the total degree of a vertex in $\widehat{G}_{1}\left[\widehat{G}_{2}\right]_{\max }$, we will use the following formula:

$$
\begin{aligned}
v_{i}-t d_{\widehat{G}_{1}\left[\widehat{G}_{2}\right]_{\max }}\left(u_{i}, v_{j}\right)= & \sum_{v_{i} v_{k} \in \hat{R}_{i}^{\prime}, w_{l} \in V_{2}} \dot{v}_{i}\left(v_{i} v_{k}\right) \\
& +\sum_{w_{j} w_{l} \in R_{i}^{\prime \prime}, v_{i}=v_{k}}\left[\dot{v}_{i}^{\prime}\left(w_{j} w_{l}\right) \vee \sigma_{1}\left(v_{i}\right)\right] \\
& +\frac{\alpha\left(v_{i}, w_{j}\right)}{n},
\end{aligned}
$$

$$
\begin{aligned}
v_{1}-t d_{\widehat{G}_{1}\left[\widehat{G}_{2}\right]_{\max }}\left(v_{1}, w_{1}\right)= & v_{1}^{\prime}\left(v_{1} v_{2}\right)+v_{1}^{\prime}\left(v_{1} v_{2}\right) \\
& +v_{1}^{\prime}\left(v_{1} v_{2}\right)+\left(v_{1}^{\prime \prime}\left(w_{1} w_{2}\right) \vee \sigma_{1}\left(v_{1}\right)\right) \\
& +\frac{\alpha\left(v_{1}, w_{1}\right)}{2} \\
= & 0.7+0.7+0.7+(0.4 \vee 0.7)+\frac{0.7}{2} \\
= & 2.1+0.7+0.35 \\
= & 3.15
\end{aligned}
$$




$$
\begin{aligned}
v_{1}-t d_{\widehat{G}_{1}\left[\widehat{G}_{2}\right]_{\max }}\left(v_{2}, w_{1}\right)= & v_{1}^{\prime}\left(v_{2} v_{1}\right)+v_{1}^{\prime}\left(v_{2} v_{1}\right)+v_{1}^{\prime}\left(v_{2} v_{1}\right) \\
& +\left(v_{1}^{\prime \prime}\left(w_{1} w_{2}\right) \vee \alpha_{1}\left(v_{2}\right)\right)+\frac{\sigma\left(v_{2}, w_{1}\right)}{2} \\
= & 0.7+0.7+0.7+(0.4 \vee 0.9)+\frac{0.9}{2} \\
= & 2.1+0.9+0.45 \\
= & 3.45,
\end{aligned}
$$

$v_{1}-t d_{\widehat{G}_{1}\left[\widehat{G}_{2}\right]_{\max }}\left(v_{1}, w_{2}\right)=v_{1}^{\prime}\left(v_{1} v_{2}\right)+v_{1}^{\prime}\left(v_{1} v_{2}\right)+v_{1}^{\prime}\left(v_{1} v_{2}\right)$

$$
\begin{aligned}
& +\left(v_{1}^{\prime \prime}\left(w_{2} w_{1}\right) \vee \alpha_{1}\left(v_{1}\right)\right)+\frac{\alpha\left(v_{1}, w_{2}\right)}{2} \\
= & 0.7+0.7+0.7+(0.4 \vee 0.7)+\frac{0.7}{2} \\
= & 2.1+0.7+0.35 \\
= & 3.15,
\end{aligned}
$$

$$
\begin{aligned}
v_{1}-t d_{\widehat{G}_{1}\left[\widehat{G}_{2}\right]_{\max }}\left(v_{2}, w_{2}\right)= & v_{1}^{\prime}\left(v_{2} v_{1}\right)+v_{1}^{\prime}\left(v_{2} v_{1}\right)+v_{1}^{\prime}\left(v_{2} v_{1}\right) \\
& +\left(v_{1}^{\prime \prime}\left(w_{2} w_{1}\right) \vee \alpha_{1}\left(v_{2}\right)\right)+\frac{\alpha\left(v_{2}, w_{2}\right)}{2} \\
= & 0.7+0.7+0.7+(0.4 \vee 0.9)+\frac{0.9}{2} \\
= & 2.1+0.9+0.45 \\
= & 3.45
\end{aligned}
$$

$$
\begin{aligned}
v_{2}-t d_{\widehat{G}_{1}\left[\widehat{G}_{2}\right]_{\max }\left(v_{4}, w_{3}\right)=} & v_{2}^{\prime}\left(v_{4} v_{3}\right)+v_{2}^{\prime}\left(v_{4} v_{3}\right) \\
& +v_{2}^{\prime}\left(v_{4} v_{3}\right)+\left(v_{2}^{\prime \prime}\left(w_{3} w_{2}\right) \vee \alpha_{1}\left(v_{4}\right)\right) \\
& +\frac{\alpha\left(v_{4}, w_{3}\right)}{2} \\
= & 0.5+0.5+0.5+(0.5 \vee 0.7)+\frac{0.7}{2} \\
= & 1.5+0.7+0.35 \\
= & 2.55
\end{aligned}
$$

$$
\begin{aligned}
v_{2}-t d_{\widehat{G}_{1}\left[\widehat{G}_{2}\right]_{\max }}\left(v_{3}, w_{3}\right)= & v_{2}^{\prime}\left(v_{3} v_{4}\right)+v_{2}^{\prime}\left(v_{3} v_{4}\right)+v_{2}^{\prime}\left(v_{3} v_{4}\right) \\
& +\left(v_{2}^{\prime \prime}\left(w_{3} w_{2}\right) \vee \alpha_{1}\left(v_{3}\right)\right)+\frac{\alpha\left(v_{3}, w_{3}\right)}{2} \\
= & 0.5+0.5+0.5+(0.5 \vee 0.5)+\frac{0.6}{2} \\
= & 1.5+0.5+0.3 \\
= & 2.3,
\end{aligned}
$$

$$
\begin{aligned}
& v_{2}-t{\widehat{G_{1}}\left[\widehat{G}_{2}\right]_{\max }\left(v_{3}, w_{2}\right)=} v_{2}^{\prime}\left(v_{3} v_{4}\right)+v_{2}^{\prime}\left(v_{3} v_{4}\right) \\
& \\
&+v_{2}^{\prime}\left(v_{3} v_{4}\right)+\left(v_{2}^{\prime \prime}\left(w_{2} w_{3}\right) \vee \alpha_{1}\left(v_{3}\right)\right) \\
&+\frac{\alpha\left(v_{3}, w_{2}\right)}{2} \\
&= 0.5+0.5+0.5+(0.5 \vee 0.5)+\frac{0.5}{2} \\
&= 1.5+0.5+0.25 \\
&= 2.25
\end{aligned}
$$

$$
\begin{aligned}
v_{2}-t{\widehat{\widehat{G}_{1}\left[\widehat{G}_{2}\right]_{\max }}}\left(v_{4}, w_{2}\right)= & v_{2}^{\prime}\left(v_{4} v_{3}\right)+v_{2}^{\prime}\left(v_{4} v_{3}\right) \\
& +v_{2}^{\prime}\left(v_{4} v_{3}\right)+\left(v_{2}^{\prime \prime}\left(w_{2} w_{3}\right) \vee \alpha_{1}\left(v_{4}\right)\right) \\
& +\frac{\alpha\left(v_{4}, w_{2}\right)}{2} \\
= & 0.5+0.5+0.5+(0.5 \vee 0.7)+\frac{0.7}{2} \\
= & 1.5+0.7+0.35 \\
= & 2.55
\end{aligned}
$$

$$
\begin{aligned}
v_{1}-t{\widehat{G_{1}}\left[\widehat{G}_{2}\right]_{\max }}\left(v_{1}, w_{3}\right)= & v_{1}^{\prime}\left(v_{1} v_{2}\right)+v_{1}^{\prime}\left(v_{1} v_{2}\right) \\
& +v_{1}^{\prime}\left(v_{1} v_{2}\right)+\frac{\sigma\left(v_{1}, w_{3}\right)}{2} \\
= & 0.7+0.7+0.7+\frac{0.7}{2} \\
= & 2.1+0.35 \\
= & 2.45,
\end{aligned}
$$

$$
\begin{aligned}
v_{1}-t d_{\widehat{G}_{1}\left[\widehat{G}_{2}\right]_{\max }}\left(v_{2}, w_{3}\right)= & v_{1}^{\prime}\left(v_{2} v_{1}\right)+v_{1}^{\prime}\left(v_{2} v_{1}\right) \\
& +v_{1}^{\prime}\left(v_{2} v_{1}\right)+\frac{\alpha\left(v_{2}, w_{3}\right)}{2} \\
= & 0.7+0.7+0.7+\frac{0.9}{2} \\
= & 2.1+0.45 \\
= & 2.55,
\end{aligned}
$$

$$
\begin{aligned}
v_{2}-t d_{\widehat{G}_{1}\left[\widehat{G}_{2}\right]_{\max }\left(v_{4}, w_{1}\right)=} & v_{2}^{\prime}\left(v_{4} v_{3}\right)+v_{2}^{\prime}\left(v_{4} v_{3}\right) \\
& +v_{2}^{\prime}\left(v_{4} v_{3}\right)+\frac{\alpha\left(v_{4}, w_{1}\right)}{2} \\
= & 0.5+0.5+0.5+\frac{0.7}{2} \\
= & 1.5+0.35 \\
= & 1.85,
\end{aligned}
$$




$$
\begin{aligned}
v_{2}-t{\widehat{G_{1}}}_{\left.\widehat{G}_{2}\right]_{\max }}\left(v_{3}, w_{1}\right)= & v_{2}^{\prime}\left(v_{3} v_{4}\right)+v_{2}^{\prime}\left(v_{3} v_{4}\right) \\
& +v_{2}^{\prime}\left(v_{3} v_{4}\right)+\frac{\alpha\left(v_{3}, w_{1}\right)}{2} \\
= & 0.5+0.5+0.5+\frac{0.5}{2} \\
= & 1.5+0.25 \\
= & 1.75,
\end{aligned}
$$

$$
\begin{aligned}
v_{1}-t{\widehat{G_{1}}\left[\widehat{G}_{2}\right]_{\max }}\left(v_{4}, w_{2}\right) & =v_{1}^{\prime \prime}\left(w_{2} w_{1}\right) \vee \alpha_{1}\left(v_{4}\right)+\frac{\alpha\left(v_{4}, w_{2}\right)}{2} \\
& =(0.4 \vee 0.7)+\frac{0.7}{2} \\
& =0.7+0.35 \\
& =1.05
\end{aligned}
$$

$$
\begin{aligned}
& v_{1}-t{\widehat{G_{1}}\left[\widehat{G}_{2}\right]_{\max }\left(v_{3}, w_{2}\right)}=v_{1}^{\prime \prime}\left(w_{2} w_{1}\right) \vee \alpha_{1}\left(v_{3}\right)+\frac{\alpha\left(v_{3}, w_{2}\right)}{2} \\
&=(0.4 \vee 0.5)+\frac{0.5}{2} \\
&=0.5+0.25 \\
&=0.75
\end{aligned}
$$

$$
\begin{aligned}
& v_{1}-t{\widehat{G_{1}}\left[\widehat{G}_{2}\right]_{\max }\left(v_{3}, w_{1}\right)}=v_{1}^{\prime \prime}\left(w_{1} w_{2}\right) \vee \alpha_{1}\left(v_{3}\right)+\frac{\alpha\left(v_{3}, w_{1}\right)}{2} \\
&=(0.4 \vee 0.5)+\frac{0.5}{2} \\
&=0.5+0.25 \\
&=0.75
\end{aligned}
$$

$$
\begin{aligned}
v_{1}-t d_{\widehat{G}_{1}\left[\widehat{G}_{2}\right]_{\max }}\left(v_{4}, w_{1}\right) & =v_{1}^{\prime \prime}\left(w_{1} w_{2}\right) \vee \alpha_{1}\left(v_{4}\right)+\frac{\alpha\left(v_{4}, w_{1}\right)}{2} \\
& =(0.4 \vee 0.7)+\frac{0.7}{2} \\
& =0.7+0.35 \\
& =1.05
\end{aligned}
$$

$$
\begin{aligned}
& v_{2}-t{\widehat{G_{1}}\left[\widehat{G}_{2}\right]_{\max }\left(v_{2}, w_{2}\right)}=v_{2}^{\prime \prime}\left(w_{2} w_{3}\right) \vee \alpha_{1}\left(v_{2}\right)+\frac{\alpha\left(v_{2}, w_{2}\right)}{2} \\
&=(0.5 \vee 0.9)+\frac{0.9}{2} \\
&=0.9+0.45 \\
&=1.35
\end{aligned}
$$

$$
\begin{aligned}
v_{2}-t d_{\widehat{G}_{1}\left[\widehat{G}_{2}\right]_{\max }\left(v_{1}, w_{2}\right)=} & v_{2}^{\prime \prime}\left(w_{2} w_{3}\right) \vee \alpha_{1}\left(v_{1}\right) \\
& +\frac{\sigma\left(v_{1}, w_{2}\right)}{2} \\
= & (0.5 \vee 0.7)+\frac{0.7}{2} \\
= & 0.7+0.35 \\
= & 1.05,
\end{aligned}
$$

$$
\begin{aligned}
v_{2}-t d_{\widehat{G}_{1}\left[\widehat{G}_{2}\right]_{\max }}\left(v_{2}, w_{3}\right)= & v_{2}^{\prime \prime}\left(w_{3} w_{2}\right) \vee \alpha_{1}\left(v_{2}\right) \\
& +\frac{\alpha\left(v_{2}, w_{3}\right)}{2} \\
= & (0.5 \vee 0.9)+\frac{0.9}{2} \\
= & 0.9+0.45 \\
= & 1.35,
\end{aligned}
$$

$$
\begin{aligned}
v_{2}-t{\widehat{G_{1}}}_{\left.\widehat{G}_{2}\right]_{\max }}\left(v_{1}, w_{3}\right)= & v_{2}^{\prime \prime}\left(w_{3} w_{2}\right) \vee \alpha_{1}\left(v_{1}\right) \\
& +\frac{\alpha\left(v_{1}, w_{3}\right)}{2} \\
= & (0.5 \vee 0.7)+\frac{0.7}{2} \\
= & 0.7+0.35 \\
= & 1.05,
\end{aligned}
$$

$$
\begin{aligned}
& v_{1}-t d_{\widehat{G}_{1}\left[\widehat{G}_{2}\right]_{\max }}\left(v_{4}, w_{3}\right)=\frac{\alpha\left(v_{4}, w_{3}\right)}{2} \\
& v_{1}-t d_{\widehat{G}_{1}\left[\widehat{G}_{2}\right]_{\max }}\left(v_{3}, w_{3}\right)=\frac{\alpha\left(v_{3}, w_{3}\right)}{2} \\
& v_{2}-t d_{\widehat{G}_{1}\left[\widehat{G}_{2}\right]_{\max }}\left(v_{1}, w_{1}\right)=\frac{\alpha\left(v_{1}, w_{1}\right)}{2} \\
& v_{2}-t d_{\widehat{G}_{1}\left[\widehat{G}_{2}\right]_{\max }}\left(v_{2}, w_{1}\right)=\frac{\alpha\left(v_{2}, w_{1}\right)}{2}
\end{aligned}
$$

Remark 3. The total degree of each vertex in $\widehat{G}_{1}\left[\widehat{G}_{2}\right]_{\max }$ is equal to the sum of its $v_{i}$-total degrees, that is,

$$
\begin{aligned}
& t d_{\widehat{G}_{1}}\left[\widehat{G}_{2}\right]_{\max }\left(u_{i}, v_{j}\right) \\
& \quad=\sum_{i=1}^{n}\left[v_{i}-t d_{\widehat{G}_{1}\left[\widehat{G}_{2}\right]_{\max }}\left(u_{i}, v_{j}\right)\right] .
\end{aligned}
$$

Example 7. It is clear from Example 6 that 


$$
\begin{aligned}
& {\left[v_{1}-t d_{\widehat{G}_{1}\left[\widehat{G}_{2}\right]_{\max }}\left(v_{1}, w_{1}\right)\right]+\left[v_{2}-t d_{\widehat{G}_{1}\left[\widehat{G}_{2}\right]_{\max }}\left(v_{1}, w_{1}\right)\right]=3.15+0.35=3.5=t d_{\widehat{G}_{1}\left[\widehat{G}_{2}\right]_{\max }}\left(v_{1}, w_{1}\right) \text {, }} \\
& {\left[v_{1}-t d_{\widehat{G}_{1}\left[\widehat{G}_{2}\right]_{\max }}\left(v_{1}, w_{2}\right)\right]+\left[v_{2}-t d_{\widehat{G}_{1}\left[\widehat{G}_{2}\right]_{\max }}\left(v_{1}, w_{2}\right)\right]=3.15+1.05=4.2=t d_{\widehat{G}_{1}\left[\widehat{G}_{2}\right]_{\max }}\left(v_{1}, w_{2}\right),} \\
& {\left[v_{1}-t d_{\widehat{G}_{1}\left[\widehat{G}_{2}\right]_{\max }}\left(v_{1}, w_{3}\right)\right]+\left[v_{2}-t d_{\widehat{G}_{1}\left[\widehat{G}_{2}\right]_{\max }}\left(v_{1}, w_{3}\right)\right]=2.45+1.05=3.5=t d_{\widehat{G}_{1}\left[\widehat{G}_{2}\right]_{\max }}\left(v_{1}, w_{3}\right) \text {, }} \\
& {\left[v_{1}-t d_{\widehat{G}_{1}}\left[\left[\widehat{G}_{2}\right]\right]_{\max }\left(v_{2}, w\right)_{1}\right]+\left[v_{2}-t d_{\widehat{G}_{1}\left[\widehat{G}_{2}\right]_{\max }}\left(v_{2}, w_{1}\right)\right]=3.45+0.45=3.9=t d_{\widehat{G}_{1}}\left[\widehat{G}_{2}\right]_{\max }\left(v_{2}, w_{1}\right) \text {, }} \\
& {\left[v_{1}-t d_{\widehat{G}_{1}\left[\widehat{G}_{2}\right]_{\max }}\left(v_{2}, w_{2}\right)\right]+\left[v_{2}-t d_{\widehat{G}_{1}\left[\widehat{G}_{2}\right]_{\max }}\left(v_{2}, w_{2}\right)\right]=3.45+1.35=4.8=t d_{\widehat{G}_{1}\left[\widehat{G}_{2}\right]_{\max }}\left(v_{2}, w_{2}\right),} \\
& {\left[v_{1}-t d_{\widehat{G}_{1}\left[\widehat{G}_{2}\right]_{\max }}\left(v_{2}, w_{3}\right)\right]+\left[v_{2}-t d_{\widehat{G}_{1}\left[\widehat{G}_{2}\right]_{\max }}\left(v_{2}, w_{3}\right)\right]=2.55+1.35=3.9=t d_{\widehat{G}_{1}\left[\widehat{G}_{2}\right]_{\max }}\left(v_{2}, w_{3}\right),} \\
& {\left[v_{1}-t d_{\widehat{G}_{1}\left[\widehat{G}_{2}\right]_{\max }}\left(v_{3}, w_{1}\right)\right]+\left[v_{2}-t d_{\widehat{G}_{1}\left[\widehat{G}_{2}\right]_{\max }}\left(v_{3}, w_{1}\right)\right]=0.75+1.75=2.5=t d_{\widehat{G}_{1}\left[\widehat{G}_{2}\right]_{\max }}\left(v_{3}, w_{1}\right),} \\
& {\left[v_{1}-t d_{\widehat{G}_{1}\left[\widehat{G}_{2}\right]_{\max }}\left(v_{3}, w_{2}\right)\right]+\left[v_{2}-t d_{\widehat{G}_{1}\left[\widehat{G}_{2}\right]_{\max }}\left[v_{3}, w_{2}\right]\right]=0.75+2.25=3.0=t d_{\widehat{G}_{1}\left[\widehat{G}_{2}\right]_{\max }}\left(v_{3}, w_{2}\right) \text {, }} \\
& {\left[v_{1}-t d_{\widehat{G}_{1}\left[\widehat{G}_{2}\right]_{\max }}\left(v_{4}, w_{1}\right)\right]+\left[v_{2}-t d_{\widehat{G}_{1}\left[\widehat{G}_{2}\right]_{\max }}\left(v_{4}, w_{1}\right)\right]=1.05+1.85=2.9=t d_{\widehat{G}_{1}\left[\widehat{G}_{2}\right]_{\max }}\left(v_{4}, w_{1}\right) \text {, }} \\
& {\left[v_{1}-t d_{\widehat{G}_{1}\left[\widehat{G}_{2}\right]_{\max }}\left(v_{4}, w_{2}\right)\right]+\left[v_{2}-t d_{\widehat{G}_{1}\left[\widehat{G}_{2}\right]_{\max }}\left(v_{4}, w_{2}\right)\right]=1.05+2.55=3.6=t d_{\widehat{G}_{1}\left[\widehat{G}_{2}\right]_{\max }}\left(v_{4}, w_{2}\right) \text {, }} \\
& {\left[v_{1}-t d_{\widehat{G}_{1}\left[\widehat{G}_{2}\right]_{\max }}\left(v_{4}, w_{3}\right)\right]+\left[v_{2}-t d_{\widehat{G}_{1}\left[\widehat{G}_{2}\right]_{\max }}\left(v_{4}, w_{3}\right)\right]=0.35+2.55=2.9=t d_{\widehat{G}_{1}\left[\widehat{G}_{2}\right]_{\max }}\left(v_{4}, w_{3}\right) \text {, }} \\
& {\left[v_{1}-t d_{\widehat{G}_{1}\left[\widehat{G}_{2}\right]_{\max }}\left(\left(v_{3}, w_{3}\right)\right]+\left[v_{2}-t d_{\widehat{G}_{1}\left[\widehat{G}_{2}\right]_{\max }}\left(v_{3}, w_{3}\right)\right]=0.3+2.3=2.6=t d_{\widehat{G}_{1}\left[\widehat{G}_{2}\right]_{\max }}\left(v_{3}, w_{3}\right) .\right.} \\
& t d_{\widehat{G}_{1}\left[\widehat{G}_{2}\right]_{\max }}\left(v_{1}, v_{2}\right)=t d_{\widehat{G}_{1}\left[\widehat{G}_{2}\right]_{\max }}\left(w_{1}, w_{2}\right), \\
& t d_{\widehat{G}_{2}}\left(v_{2}\right)+t d_{\widehat{G}_{1}}\left(v_{1}\right)\left|V_{2}\right|=t d_{\widehat{G}_{2}}\left(w_{2}\right)+t d_{\widehat{G}_{1}}\left(w_{1}\right)\left|V_{2}\right| \text {. }
\end{aligned}
$$
$\widehat{G}_{1}\left[\widehat{G}_{2}\right]_{\max }$ is equal to the sum of its $\nu_{i}$-total degrees.

Theorem 11. If $\widehat{G}_{1}$ and $\widehat{G}_{2}$ are two FGSs such that $\nu_{i}^{\prime \prime} \geq \alpha_{1}$, then the lexicographic-max product $\widehat{G}_{1}\left[\widehat{G}_{2}\right]_{\max }$ is a totally regular FGS if and only if $\widehat{G}_{1}$ and $\widehat{G}_{2}$ are totally regular FGSs.

Proof. Let $\widehat{G}_{1}=\left(\alpha_{1}, v_{1}^{\prime}, v_{2}^{\prime}, \ldots, v_{n}^{\prime}\right)$ and $\widehat{G}_{2}=\left(\alpha_{2}, v_{1}^{\prime \prime}, v_{2}^{\prime \prime}, \ldots, v_{n}^{\prime \prime}\right)$ be two FGSs such that $\gamma_{i}^{\prime \prime} \geq \alpha_{1}$. Then, the total degree of the vertex in the lexicographic-max product $\widehat{G}_{1}\left[\widehat{G}_{2}\right]_{\max }$ is given by

$$
t d_{\widehat{G}_{1}\left[\widehat{G}_{2}\right]_{\max }}\left(v_{i}, w_{j}\right)=t d_{\widehat{G}_{2}}\left(w_{j}\right)+t d_{\widehat{G}_{1}}\left(v_{i}\right)\left|V_{2}\right| .
$$

Assuming that $\widehat{G}_{1}=\left(\alpha_{1}, v_{1}^{\prime}, v_{2}^{\prime}, \ldots, v_{n}^{\prime}\right)$ and $\widehat{G}_{2}=\left(\alpha_{2}, v_{1}^{\prime \prime}\right.$, $\left.v_{2}^{\prime \prime}, \ldots, v_{n}^{\prime \prime}\right)$ are two totally regular FGSs of degree $k_{1}$ and $k_{2}$, respectively, then

$$
t d_{\widehat{G}_{1}\left[\widehat{G}_{2}\right]_{\max }}\left(v_{i}, w_{j}\right)=k_{2}+k_{1}\left|V_{2}\right| .
$$

Since $k_{1}, k_{2}$, and $\left|V_{2}\right|$ are constants, so $d_{\left.\widehat{G}_{[} \widehat{G}_{2}\right]}$ is a constant for all elements of $V_{1} \times V_{2}$. Thus, the lexicographic max-product $\widehat{G}_{1}\left[\widehat{G}_{2}\right]_{\max }=\left(\alpha, v_{1}, v_{2}, \ldots, v_{n}\right)$ is a totally regular FGS.

Conversely, suppose that $\widehat{G}_{1}=\left(\alpha_{1}, v_{1}^{\prime}, v_{2}^{\prime}, \ldots, v_{n}^{\prime}\right)$ and $\widehat{G}_{2}=\left(\alpha_{2}, v_{1}^{\prime \prime}, v_{2}^{\prime \prime}, \ldots, v_{n}^{\prime \prime}\right)$ are two FGSs such that $v_{i}^{\prime \prime} \geq \alpha_{1}$ and the lexicographic-max product $\widehat{G}_{1}\left[\widehat{G}_{2}\right]_{\max }=\left(\alpha, \nu_{1}, \nu_{2}, \ldots\right.$, $\left.v_{n}\right)$ is a totally regular FGS. Then, the total degrees of two vertices $\left(v_{1}, v_{2}\right)$ and $\left(w_{1}, w_{2}\right)$ in $V_{1} \times V_{2}$ are equal, that is,
Fixing $v \in V_{2}$ and considering $\left(v_{1}, v\right)$ and $\left(w_{1}, v\right)$ in $V_{1} \times V_{2}$, where $v_{1}$ and $w_{1}$ are arbitrary elements of the vertex set $V_{1}$, then the above equation becomes

$$
\begin{aligned}
t d_{\widehat{G}_{2}}(v)+t d_{\widehat{G}_{1}}\left(v_{1}\right)\left|V_{2}\right| & =t d_{\widehat{G}_{2}}((v))+t d_{\widehat{G}_{1}}\left(w_{1}\right)\left|V_{2}\right|, \\
t d_{\widehat{G}_{1}}\left(v_{1}\right)\left|V_{2}\right| & =t d_{\widehat{G}_{1}}\left(w_{1}\right)\left|V_{2}\right|, \\
t d_{\widehat{G}_{1}}\left(v_{1}\right) & =t d_{\widehat{G}_{1}}\left(w_{1}\right) .
\end{aligned}
$$

This holds for all elements $v_{1}$ and $w_{1}$ of $V_{1}$. Thus, $\widehat{G}_{1}=$ $\left(\alpha_{1}, v_{1}^{\prime}, v_{2}^{\prime}, \ldots, v_{n}^{\prime}\right)$ is a totally regular FGS.

Now fixing $v / \in V_{1}$ and supposing that $\left(v \prime, v_{2}\right)$ and $\left(v /, w_{2}\right)$ in $V_{1} \times V_{2}$, where $v_{2}$ and $w_{2}$ are taken as the arbitrary vertices of the set $V_{2}$, then the above expression changes to

$$
\begin{aligned}
t d_{\widehat{G}_{2}}\left(v_{2}\right)+t d_{\widehat{G}_{1}}\left(v^{\prime}\right)\left|V_{2}\right| & =t d_{\widehat{G}_{2}}\left(w_{2}\right)+t d_{\widehat{G}_{1}}\left(v^{\prime}\right)\left|V_{2}\right|, \\
t d_{\widehat{G}_{2}}\left(v_{2}\right) & =t d_{\widehat{G}_{2}}\left(w_{2}\right) .
\end{aligned}
$$

This holds for all vertices $v_{2}$ and $w_{2}$ of $V_{2}$. Thus, $\widehat{G}_{2}=$ $\left(\alpha_{2}, v_{1}^{\prime \prime}, v_{2}^{\prime \prime}, \ldots, v_{n}^{\prime \prime}\right)$ is a totally regular FGS.

Theorem 12. If $\widehat{G}_{1}$ and $\widehat{G}_{2}$ are two FGSs such that $\nu_{i}^{\prime \prime} \geq \alpha_{1}$, then the lexicographic-max product $\widehat{G}_{1}\left[\widehat{G}_{2}\right]_{\max }$ is a regular 
FGS on complete GS if and only if $\widehat{G}_{1}$ and $\widehat{G}_{2}$ are regular FGSs on complete GSs $\widehat{G}_{1}^{*}$ and $\widehat{G}_{2}^{*}$, respectively.

Proof. The proof of Theorem 11 implies the proof of this theorem.

\section{Applications}

3.1. Detection of Marine Crimes. Marine crime is as old as this industry itself. Depending on the times that it prevailed in, the nature of the maritime crime has changed a lot over the years, but its implications remain just as severe. Marine crime is not only a threat to entire maritime security of goods and people in the industry, but also gives major setbacks to the entire marine industry economically. Marine crimes are usually held during the maritime trade. Seaports are very important in the whole world for trade through waters. There are almost four thousand seven hundred and sixty-four seaports, which are frequently used for import and export of various types of goods. Seaports have been very useful and beneficial for hundreds of years. But, they have also been used for the illegal trade due to which seaports always remain sensitive for crimes. Nowadays, crimes occurring on the seaports are in abundance, but some crimes including human trafficking, smuggling of
TABLE 1: Fuzzy set $\alpha$ of seaports.

\begin{tabular}{lc}
\hline Seaport & Degree of membership \\
\hline Mongla & 0.8 \\
Bintulu & 0.9 \\
Sembawang & 0.7 \\
Abu Dhabi & 0.8 \\
Gwadar & 0.7 \\
Chennai & 0.9 \\
Malindi & 0.8 \\
Ciro Marina & 0.5 \\
\hline
\end{tabular}

precious metals, illegal carrying of weapons and artillery, smuggling of exotic plants, trade of drugs, and smuggling of exotic animals are crucial. All these crimes pose threat to the maritime security on a daily basis. Security of waters is still suffering because this is a very broad aspect without concrete boundaries. A fuzzy graph structure of marine crimes occurring on seaports can be very helpful to combat these crimes. Using the fuzzy graph structure, we can investigate that between any two seaports which crime is chronic and increasing violently. Moreover, we can decide which seaport is the most sensitive for a particular crime and needed to be focused by security teams. Consider a set $V$ of eight seaports:

$$
V=\{\text { Mongla, Bintulu, Sembawang, Abu Dhabi, Gwadar, Chennai, Malindi, Ciro Marina }\}
$$

Let $\alpha$ be defined as a fuzzy set on $V$, as given in Table 1 . In Table 1, the membership value of a seaport depicts its importance in the world due to its geographic position.

In Tables 2-11, we have mentioned membership values of marine crimes among each pair of seaports. The membership value of each pair of seaports is calculated according to $v\left(v_{1} v_{2}\right) \leq \alpha\left(v_{1}\right) \wedge \alpha\left(v_{2}\right)$, for all $v_{1}, v_{2} \in V$. By using these membership values, we show the severity level of each marine crime among each pair of seaports.

On the set $V$, many relations can be defined; let us define the following relations on $V . R_{1}=$ human trafficking, $R_{2}=$ illegal carrying of weapons and artillery, $R_{3}=$ smuggling of exotic plants, $R_{4}=$ smuggling of precious metals,
$R_{5}=$ trade of drugs, and $R_{6}=$ smuggling of exotic animals such that $\left(V, R_{1}, R_{2}, R_{3}, R_{4}, R_{5}, R_{6}\right)$ is a graph structure. Each element in the relation depicts a particular kind of the most crucial marine crimes among those two seaports.

As $\left(V, R_{1}, R_{2}, R_{3}, R_{4}, R_{5}, R_{6}\right)$ is the graph structure, therefore a pair of seaports can appear in just one relation. Hence, it would be considered an element of that relation, for which its membership value is comparatively high than that of the other relations. Using the above given data, elements in relations are paired with their membership values, and resulting sets are the fuzzy sets on $R_{1}, R_{2}, R_{3}, R_{4}, R_{5}$, and $R_{6}$, respectively. These fuzzy sets are named as $v_{1}, v_{2}, v_{3}, v_{4}$, $\nu_{5}$, and $v_{6}$, respectively. Let

$$
\begin{aligned}
& R_{1}=\{(\text { Bintulu, Mongla }),(\text { Bintulu, Gwadar }), \text { (Mongla, Chennai), (Abu Dhabi, Mongla }), \\
& R_{2}=(\{\text { Abu Dhabi, Bintulu }\}), \\
& R_{3}=\{(\text { Abu Dhabi, Malindi), (Ciro Marina, Malindi })\}, \\
& R_{4}=\{(\text { Bintulu, Chennai), (Sembawang, Mongla })\}, \\
& R_{5}=\{(\text { Gwadar, Malindi), (Ciro Marina, Sembawang })\}, \\
& R_{6}=\{(\text { Bintulu, Ciro Marina })\}
\end{aligned}
$$


TABLe 2: Fuzzy set of marine crimes among Gwadar and other seaports.

\begin{tabular}{lcccc}
\hline Type of marine crimes & (Gwadar, Chennai) & (Gwadar, Malindi) & (Gwadar, Ciro Marina) & (Gwadar, Mongla) \\
\hline Human trafficking & 0.7 & 0.6 & 0.4 & 0.1 \\
Illegal carrying of weapons and artillery & 0.2 & 0.1 & 0.2 & 0.2 \\
Smuggling of exotic plants & 0.1 & 0.4 & 0.2 & 0.1 \\
Smuggling of precious metals & 0.1 & 0.1 & 0.3 & 0.3 \\
Trade of drugs & 0.2 & 0.7 & 0.3 & 0.5 \\
Smuggling of exotic animals & 0.2 & 0.2 & 0.1 \\
\hline
\end{tabular}

Table 3: Fuzzy set of marine crimes among Malindi and other seaports.

\begin{tabular}{lcccc}
\hline Type of marine crimes & (Malindi, Mongla) & (Malindi, Bintulu) & (Malindi, Sembawang) & (Malindi, Chennai) \\
\hline Human trafficking & 0.8 & 0.2 & 0.5 & 0.7 \\
Illegal carrying of weapons and artillery & 0.6 & 0.3 & 0.4 & 0.6 \\
Smuggling of exotic plants & 0.1 & 0.2 & 0.3 & 0.4 \\
Smuggling of precious metals & 0.6 & 0.8 & 0.3 & 0.6 \\
Trade of drugs & 0.2 & 0.5 & 0.4 & 0.5 \\
Smuggling of exotic animals & 0.3 & 0.3 & & 0.4 \\
\hline
\end{tabular}

TABle 4: Fuzzy set of marine crimes among Ciro Marina and other seaports.

\begin{tabular}{lccc}
\hline Type of marine crimes & (Ciro Marina, Sembawang) & (Ciro Marina, Abu Dhabi) & (Ciro Marina, Bintulu) \\
\hline Human trafficking & 0.1 & 0.1 & 0.1 \\
Illegal carrying of weapons and artillery & 0.1 & 0.2 & 0.1 \\
Smuggling of exotic plants & 0.2 & 0.5 & 0.0 \\
Smuggling of precious metals & 0.1 & 0.2 & 0.2 \\
Trade of drugs & 0.5 & 0.2 & 0.4 \\
Smuggling of exotic animals & 0.2 & 0.1 & 0.5 \\
\hline
\end{tabular}

Table 5: Fuzzy set of marine crimes among Sembawang and other seaports.

\begin{tabular}{lccc}
\hline Type of marine crimes & (Sembawang, Gwadar) & (Sembawang, Chennai) & (Sembawang, Mongla) \\
\hline Human trafficking & 0.1 & 0.2 & 0.6 \\
Illegal carrying of weapons and artillery & 0.2 & 0.3 & 0.4 \\
Smuggling of exotic plants & 0.2 & 0.2 & 0.6 \\
Smuggling of precious metals & 0.1 & 0.2 & 0.7 \\
Trade of drugs & 0.7 & 0.7 & 0.4 \\
Smuggling of exotic animals & 0.3 & 0.6 \\
\hline
\end{tabular}

Table 6: Fuzzy set of marine crimes among Mongla and other seaports.

\begin{tabular}{lccc}
\hline Type of marine crimes & (Mongla, Bintulu) & (Mongla, Sembawang) & (Mongla, Abu Dhabi) \\
\hline Human trafficking & 0.8 & 0.5 & 0.8 \\
Illegal carrying of weapons and artillery & 0.6 & 0.2 & 0.4 \\
Smuggling of exotic plants & 0.1 & 0.6 & 0.3 \\
Smuggling of precious metals & 0.7 & 0.7 & 0.4 \\
Trade of drugs & 0.5 & 0.5 & 0.6 \\
Smuggling of exotic animals & 0.3 & 0.6 & 0.4 \\
\hline
\end{tabular}

TABle 7: Fuzzy set of marine crimes among Chennai and other seaports.

\begin{tabular}{lccc}
\hline Type of marine crimes & (Chennai, Mongla) & (Chennai, Malindi) & (Chennai, Ciro Marina) \\
\hline Human trafficking & 0.8 & 0.5 & 0.2 \\
Illegal carrying of weapons and artillery & 0.6 & 0.6 & 0.2 \\
Smuggling of exotic plants & 0.2 & 0.6 & 0.1 \\
Smuggling of precious metals & 0.6 & 0.5 & 0.2 \\
Trade of drugs & 0.5 & 0.5 & 0.4 \\
Smuggling of exotic animals & 0.6 & 0.5 & 0.5 \\
\hline
\end{tabular}


TABle 8: Fuzzy set of marine crimes among Abu Dhabi and other seaports.

\begin{tabular}{lccc}
\hline Type of marine crimes & (Abu Dhabi, Gwadar) & (Abu Dhabi, Chennai) & (Abu Dhabi, Malindi) \\
\hline Human trafficking & 0.3 & 0.8 & 0.5 \\
Illegal carrying of weapons and artillery & 0.2 & 0.6 & 0.4 \\
Smuggling of exotic plants & 0.5 & 0.5 & 0.8 \\
Smuggling of precious metals & 0.6 & 0.4 & 0.7 \\
Trade of drugs & 0.7 & 0.3 & 0.6 \\
Smuggling of exotic animals & 0.3 & 0.6 \\
\hline
\end{tabular}

TABLE 9: Fuzzy set of marine crimes among some seaports.

\begin{tabular}{lccc}
\hline Type of marine crimes & (Mongla, Gwadar) & (Ciro Marina, Malindi) & (Sembawang, Abu Dhabi) \\
\hline Human trafficking & 0.1 & 0.1 & 0.5 \\
Illegal carrying of weapons and artillery & 0.2 & 0.2 & 0.4 \\
Smuggling of exotic plants & 0.2 & 0.5 & 0.2 \\
Smuggling of precious metals & 0.4 & 0.1 & 0.4 \\
Trade of drugs & 0.7 & 0.3 & 0.7 \\
Smuggling of exotic animals & 0.3 & 0.2 & 0.2 \\
\hline
\end{tabular}

TABle 10: Fuzzy set of marine crimes among Bintulu and other seaports.

\begin{tabular}{lccc}
\hline Type of marine crimes & (Bintulu, Mongla) & (Bintulu, Sembawang) & (Bintulu, Abu Dhabi) \\
\hline Human trafficking & 0.8 & 0.5 & 0.7 \\
Illegal carrying of weapons and artillery & 0.6 & 0.4 & 0.8 \\
Smuggling of exotic plants & 0.1 & 0.1 & 0.3 \\
Smuggling of precious metals & 0.7 & 0.2 & 0.4 \\
Trade of drugs & 0.5 & 0.3 & 0.6 \\
Smuggling of exotic animals & 0.3 & 0.5 & 0.6 \\
\hline
\end{tabular}

TABLE 11: Fuzzy set of marine crimes among some seaports.

\begin{tabular}{lccc}
\hline Type of marine crimes & (Bintulu, Gwadar) & (Chennai, Bintulu) & (Abu Dhabi, Ciro Marina) \\
\hline Human trafficking & 0.7 & 0.3 & 0.3 \\
Illegal carrying of weapons and artillery & 0.6 & 0.4 & 0.2 \\
Smuggling of exotic plants & 0.2 & 0.5 & 0.5 \\
Smuggling of precious metals & 0.6 & 0.9 & 0.1 \\
Trade of drugs & 0.5 & 0.3 & 0.1 \\
Smuggling of exotic animals & 0.5 & 0.4 & 0.1 \\
\hline
\end{tabular}

And, their corresponding fuzzy sets are

$$
\begin{aligned}
& v_{1}=\{((\text { Bintulu, Mongla }), 0.8),((\text { Bintulu, Gwadar }), 0.7),((\text { Mongla, Chennai }), 0.8),((\text { Abu Dhabi, Mongla }), 0.8)\} \\
& v_{2}=\{((\text { Abu Dhabi, Bintulu }), 0.8)\} \\
& v_{3}=\{((\text { Abu Dhabi, Malindi }), 0.8),((\text { Ciro Marina, Malindi }), 0.5)\} \\
& v_{4}=\{((\text { Bintulu, Chennai }), 0.9),((\text { Sembawang, Mongla }), 0.7)\} \\
& v_{5}=\{((\text { Gwadar, Malindi }), 0.7),((\text { Ciro Marina, Sembawang }), 0.5)\} \\
& v_{6}=\{((\text { Bintulu, Ciro Marina }), 0.5)\}
\end{aligned}
$$

Clearly, $\left(\alpha, v_{1}, v_{2}, v_{3}, v_{4}, v_{5}, v_{6}\right)$ is a fuzzy graph structure and is shown in Figure 13.

In FGS shown in Figure 13, each edge depicts the most crucial marine crime among the corresponding seaports. For example, most crucial marine crime among Bintulu and
Mongla is human trafficking, and its membership value is 0.8 . It can be noted that the vertex Bintulu has the highest vertex degree for the relation human trafficking, and it means that Bintulu has human trafficking as the most crucial marine crime with other seaports. Moreover, according to 


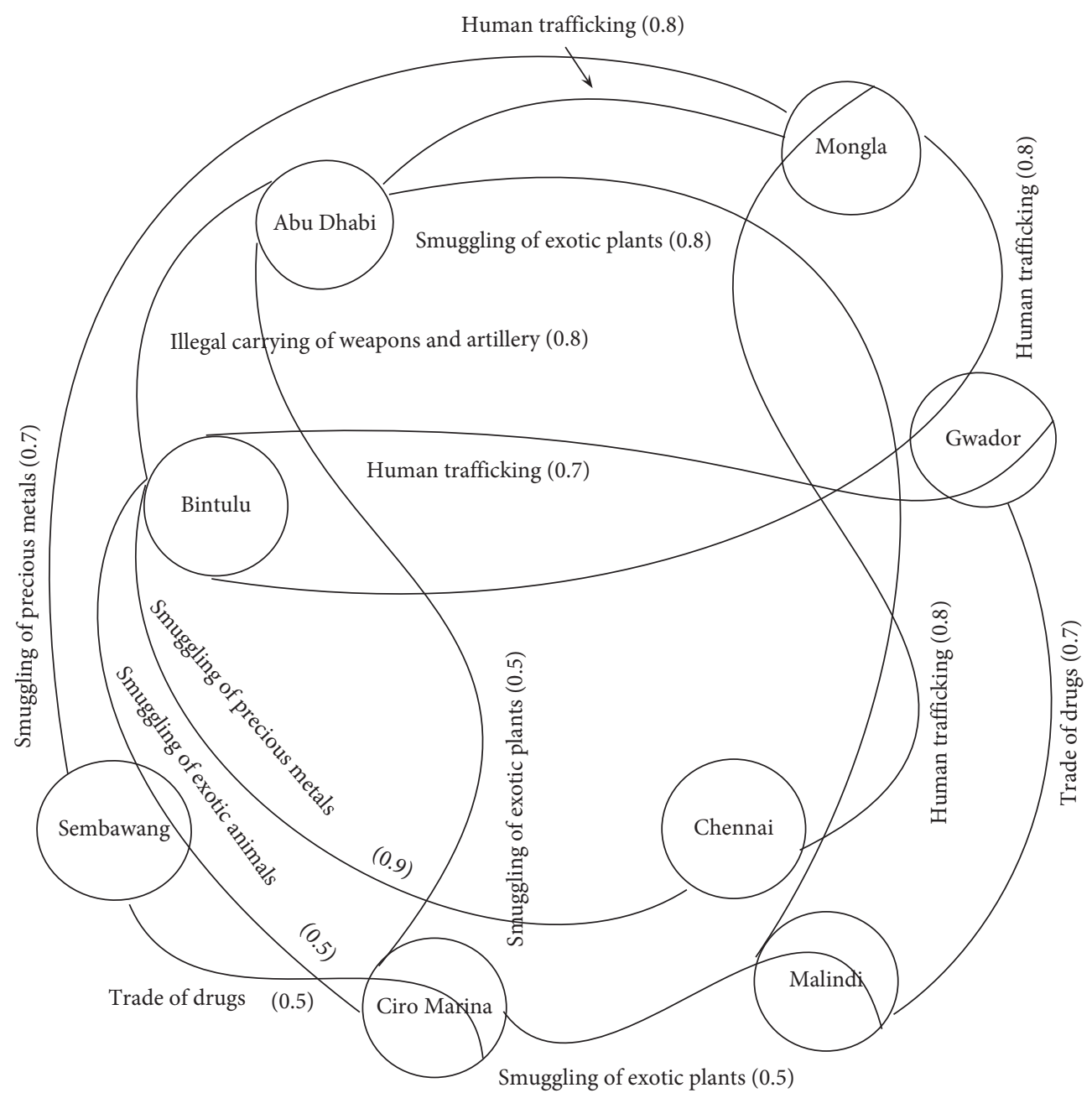

FIgURE 13: Fuzzy graph structure depicting most crucial marine crimes among different seaports.

this fuzzy graph structure, Abu Dhabi and Malindi have smuggling of exotic plants as the most crucial marine crime at this time with a membership value 0.8 . An FGS of all seaports can be very helpful for security agencies of all countries. It highlights those marine crimes which needed to be solved promptly.

3.2. Detection of Road Crimes. Road network is very important for any country. Roads connecting cities are the major source of trade, traveling, and many other purposes. But, nowadays, roads have become unsafe due to large amount of crimes happening on the roads. Crimes occurring on roads are very large in number, but some crimes are very crucial including child kidnapping, bike robbery, mobile snatching, women harassment, car robbery, and robbery of animals. Using a fuzzy graph structure, we can investigate that which road is the most crucial for a particular crime. An FGS can also tell us which crime is the most chronic and which one is increasing rapidly. Furthermore, we can judge which road is the most crucial for a particular road crime. An FGS of road crimes can be very helpful for the police department to overcome a particular crime, and it can guide them in making a policy against a crime. For example, if an
Table 12: Fuzzy set F on $A$.

\begin{tabular}{lc}
\hline City & Membership value \\
\hline Islamabad & 0.9 \\
Karachi & 0.5 \\
Rawalpindi & 0.8 \\
Lahore & 0.8 \\
Multan & 0.7 \\
Jhang & 0.8 \\
Faisalabad & 0.7 \\
Sheikhupura & 0.7 \\
\hline
\end{tabular}

FIR is written of a crime held in a particular area, an FGS of that area can be very helpful for the police department in taking action against that crime, and it will guide them to which roads are the most sensitive regarding that particular crime. So, police can put check posts on those roads, and police can cordon off the area near to those roads. Consider a set $A$ of eight cities. $A=\{$ Islamabad, Karachi, Rawalpindi, Lahore, Multan, Jhang, Faisalabad, Sheikhupura\}. Let $F$ be considered as a fuzzy set on $A$, as given in Table 12 .

In Table 12, the membership value shows the solidity of that particular city corresponding to economical progress, educational and health facilities, and peace. In Tables 13-20, 
TABLE 13: Fuzzy set of crimes on roads connecting Islamabad and other cities.

\begin{tabular}{lcccc}
\hline Type of crime & (Islamabad, Karachi) & (Islamabad, Rawalpindi) & (Islamabad, Lahore) & (Islamabad, Multan) \\
\hline Child kidnapping & 0.6 & 0.5 & 0.5 & 0.7 \\
Bike robbery & 0.6 & 0.6 & 0.4 & 0.6 \\
Mobile snatching & 0.5 & 0.6 & 0.4 & 0.5 \\
Women harassment & 0.6 & 0.5 & 0.6 & 0.6 \\
Car robbery & 0.5 & 0.5 & 0.4 & 0.5 \\
Robbery of animals & 0.3 & 0.5 & & 0.4 \\
\hline
\end{tabular}

TABle 14: Fuzzy set of crimes on roads connecting Rawalpindi and other cities.

\begin{tabular}{lcccc}
\hline Type of crime & (Rawalpindi, Lahore) & (Rawalpindi, Multan) & (Rawalpindi, Jhang) & (Rawalpindi, Islamabad) \\
\hline Child kidnapping & 0.7 & 0.6 & 0.7 & 0.7 \\
Bike robbery & 0.8 & 0.7 & 0.8 & 0.6 \\
Mobile snatching & 0.6 & 0.6 & 0.6 & 0.7 \\
Women harassment & 0.8 & 0.7 & 0.6 & 0.8 \\
Car robbery & 0.7 & 0.5 & 0.5 & 0.7 \\
Robbery of animals & 0.4 & 0.5 & 0.3 \\
\hline
\end{tabular}

TABle 15: Fuzzy set of crimes on roads connecting Faisalabad and other cities.

\begin{tabular}{lcccc}
\hline Type of crime & (Faisalabad, Islamabad) & (Faisalabad, Karachi) & (Faisalabad, Rawalpindi) & (Faisalabad, Jhang) \\
\hline Child kidnapping & 0.7 & 0.5 & 0.6 & 0.5 \\
Bike robbery & 0.5 & 0.4 & 0.4 & 0.6 \\
Mobile snatching & 0.7 & 0.5 & 0.7 & 0.6 \\
Women harassment & 0.6 & 0.4 & 0.6 & 0.5 \\
Car robbery & 0.7 & 0.5 & 0.6 & 0.6 \\
Robbery of animals & 0.3 & 0.5 & 0.7 \\
\hline
\end{tabular}

TABle 16: Fuzzy set of crimes on roads connecting Sheikhupura and other cities.

\begin{tabular}{lcccc}
\hline Type of crime & (Sheikhupura, Rawalpindi) & (Islamabad, Sheikhupura) & (Sheikhupura, Karachi) & (Sheikhupura, Faisalabad) \\
\hline Child kidnapping & 0.7 & 0.6 & 0.4 & 0.5 \\
Bike robbery & 0.6 & 0.6 & 0.4 & 0.6 \\
Mobile snatching & 0.7 & 0.5 & 0.4 & 0.7 \\
Women harassment & 0.6 & 0.6 & 0.4 & 0.5 \\
Car robbery & 0.7 & 0.7 & 0.5 & 0.4 \\
Robbery of animals & 0.6 & 0.4 & 0.4 & 0.6 \\
\hline
\end{tabular}

TABLE 17: Fuzzy set of crimes on roads connecting Lahore and other cities.

\begin{tabular}{lcccc}
\hline Type of crime & (Lahore, Multan) & (Lahore, Jhang) & (Lahore, Faisalabad) & (Lahore, Sheikhupura) \\
\hline Child kidnapping & 0.7 & 0.7 & 0.6 & 0.5 \\
Bike robbery & 0.6 & 0.8 & 0.4 & 0.7 \\
Mobile snatching & 0.6 & 0.7 & 0.4 & 0.6 \\
Women harassment & 0.5 & 0.6 & 0.6 & 0.4 \\
Car robbery & 0.6 & 0.5 & 0.5 & 0.6 \\
Robbery of animals & 0.6 & 0.6 & & 0.6 \\
\hline
\end{tabular}

TABLE 18: Fuzzy set of crimes on roads connecting Jhang and other cities.

\begin{tabular}{lcccc}
\hline Type of crime & (Jhang, Islamabad) & (Jhang, Faisalabad) & (Jhang, Sheikhupura) & (Jhang, Karachi) \\
\hline Child kidnapping & 0.7 & 0.5 & 0.6 & 0.4 \\
Bike robbery & 0.5 & 0.6 & 0.5 & 0.6 \\
Mobile snatching & 0.8 & 0.6 & 0.4 & 0.7 \\
Women harassment & 0.8 & 0.6 & 0.6 & 0.7 \\
Car robbery & 0.7 & 0.5 & 0.5 & 0.6 \\
Robbery of animals & 0.6 & 0.7 & & 0.7 \\
\hline
\end{tabular}


TABLe 19: Fuzzy set of crimes on roads connecting Multan and other cities.

\begin{tabular}{lcccc}
\hline Type of crime & (Multan, Jhang) & (Multan, Faisalabad) & (Multan, Sheikhupura) & (Multan, Islamabad) \\
\hline Child kidnapping & 0.5 & 0.5 & 0.5 & 0.6 \\
Bike robbery & 0.6 & 0.6 & 0.6 & 0.4 \\
Mobile snatching & 0.6 & 0.5 & 0.7 & 0.6 \\
Women harassment & 0.7 & 0.6 & 0.4 & 0.5 \\
Car robbery & 0.6 & 0.5 & 0.7 & 0.7 \\
Robbery of animals & 0.6 & 0.7 & 0.5 \\
\hline
\end{tabular}

Table 20: Fuzzy set of crimes on roads connecting Karachi and other cities.

\begin{tabular}{lcccc}
\hline Type of crime & (Islamabad, Multan) & (Karachi, Rawalpindi) & (Karachi, Lahore) & (Karachi, Multan) \\
\hline Child kidnapping & 0.6 & 0.4 & 0.5 & 0.4 \\
Bike robbery & 0.4 & 0.2 & 0.4 & 0.4 \\
Mobile snatching & 0.6 & 0.3 & 0.4 & 0.2 \\
Women harassment & 0.5 & 0.4 & 0.4 & 0.5 \\
Car robbery & 0.7 & 0.5 & 0.4 & 0.4 \\
Robbery of animals & 0.5 & 0.4 & & 0.3 \\
\hline
\end{tabular}

we have shown membership values of different crimes on roads connecting every pair of cities.

Various relations can be defined on the set $A$, and here we define the following six relations on $A . R_{1}=$ child kidnapping, $R_{2}$ =bike robbery, $R_{3}=$ mobile snatching, $R_{4}=$ women harassment, $R_{5}=$ car robbery, and $R_{6}=$ robbery of animals such that $\left(A, R_{1}, R_{2}, R_{3}, R_{4}, R_{5}, R_{6}\right)$ is a graph structure. Each object in an arbitrary relation shows a particular type of crime occurring on the roads connecting those two cities.
As $\left(A, R_{1}, R_{2}, R_{3}, R_{4}, R_{5}, R_{6}\right)$ is a graph structure, so an element cannot appear in more than one relation, and that is why it appears just once. Due to this, it is considered as an element of that particular relation, whose membership degree is higher than the membership values of all remaining relations.

In accordance with the above knowledge, elements in these relations are written with their corresponding membership values, and corresponding sets are fuzzy sets on $R_{1}$, $R_{2}, R_{3}, R_{4}, R_{5}$, and $R_{6}$, respectively. These sets are named as $\mu_{1}, \mu_{2}, \mu_{3}, \mu_{4}, \mu_{5}$, and $\mu_{6}$, respectively. Let

$$
\begin{aligned}
& R_{1}=\{(\text { Karachi, Lahore }),(\text { Lahore, Multan }), \\
& R_{2}=\{(\text { Jhang, Lahore), (Sheikhupura, Lahore }), \\
& R_{3}=((\text { Karachi, Multan), (Faisalabad, Lahore }),(\text { Sheikhupura, Faisalabad }), \\
& R_{4}=\{(\text { Multan, Jhang), (Faisalabad, Rawalpindi }), \\
& R_{5}=\{(\text { Islamabad, Multan), (Karachi, Rawalpindi), (Sheikhupura, Karachi), (Islamabad, Sheikhupura })\}, \\
& R_{6}=\{(\text { Multan, Faisalabad), (Jhang, Faisalabad }),
\end{aligned}
$$

And, corresponding fuzzy sets are

$\mu_{1}=\{(($ Karachi, Lahore $), 0.5),(($ Lahore, Multan $), 0.7)\}$,

$\mu_{2}=\{(($ Jhang, Lahore $), 0.8),(($ Sheikhupura, Lahore $), 0.7)\}$,

$\mu_{3}=\{(($ Karachi, Multan $), 0.5),(($ Faisalabad, Lahore $), 0.7),(($ Sheikhupura, Faisalabad) $) 0.7)\}$,

$\mu_{4}=\{(($ Multan, Jhang $), 0.7),(($ Faisalabad, Rawalpindi) $) 0.7)\}$,

$\mu_{5}=\{(($ Islamabad, Multan), 0.7), ((Karachi, Rawalpindi), 0.5), ((Sheikhupura, Karachi), 0.5), ((Islamabad, Sheikhupura), 0.7) $\}$,

$\mu_{6}=\{(($ Multan, Faisalabad), 0.7), ((Jhang, Faisalabad), 0.7) $\}$. 


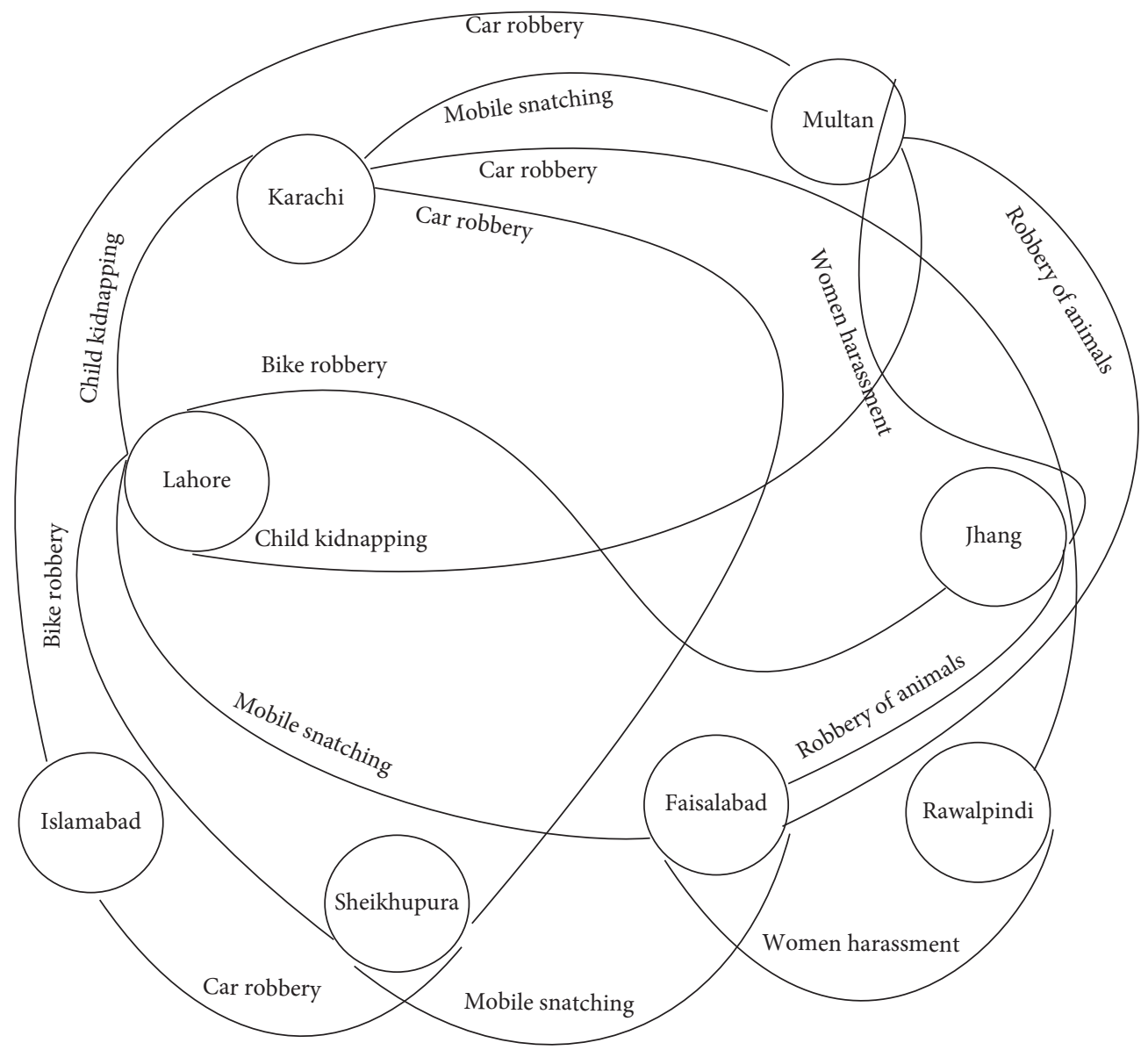

Figure 14: Fuzzy graph structure showing the most crucial crime on roads connecting any two cities.

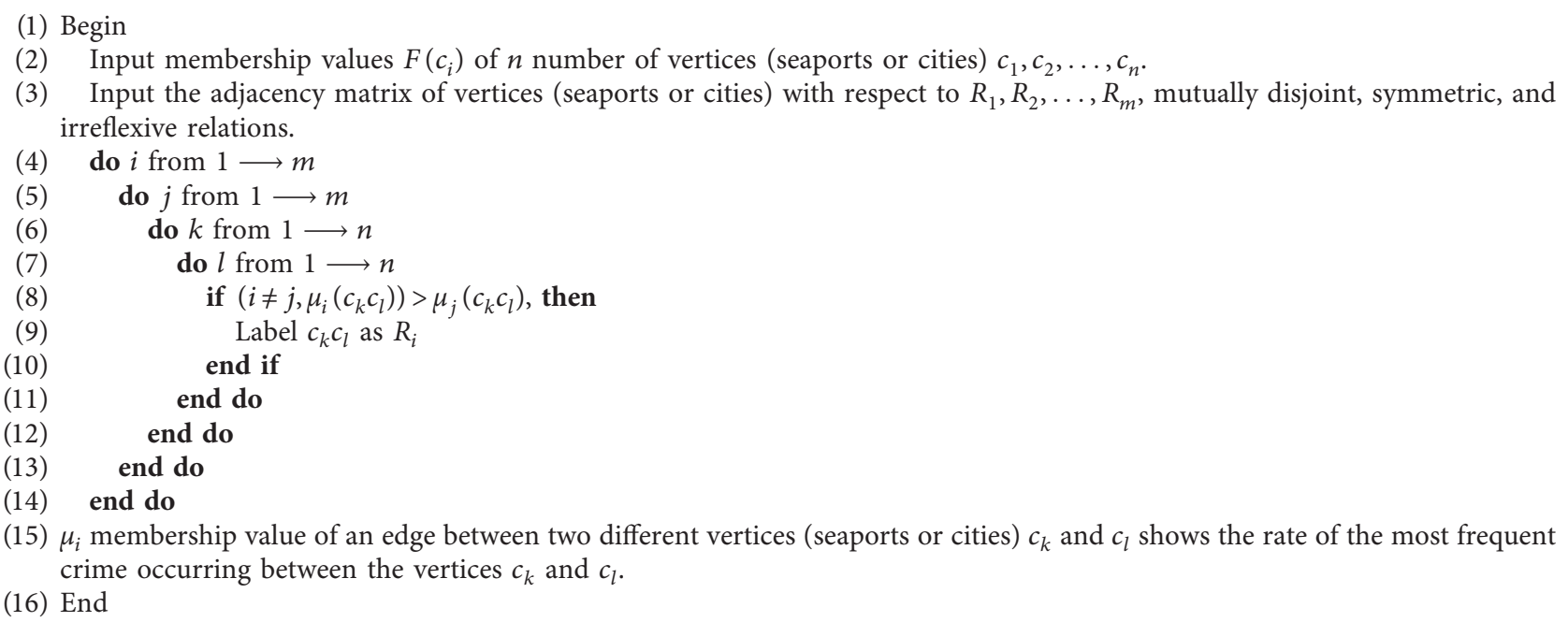

Algorithm 1: Computing membership value of an edge between two different vertices.

Clearly, $\left(F, \mu_{1}, \mu_{2}, \mu_{3}, \mu_{4}, \mu_{5}, \mu_{6}\right)$ is a fuzzy graph structure and is shown in Figure 14.

In the fuzzy graph structure shown in Figure 14, each edge shows the most frequent crime occurring on roads connecting corresponding cities. For example, most frequent crime on roads connecting Karachi and Rawalpindi is car robbery, and its membership value is 0.5 . It can be noted that the vertex Karachi has the highest vertex degree for the 
relation car robbery, and it means Karachi is the most sensitive city for robbery of cars. Moreover, according to the above fuzzy graph structure, the most frequent road crime is car robbery. Furthermore, we can also observe that the most frequent crime after car robbery is mobile snatching. It means that the police department should take actions to control car robbery and mobile snatching.

The general procedure of the adopted method used in the above applications is presented in Algorithm 1.

\section{Conclusions}

Fuzzy graph structures are more advantageous than graph structures to cope with uncertainty. In this research paper, we have combined the fruitful theories of fuzzy sets and graph structures to introduce certain notions of fuzzy graph structures. We have introduced the lexicographic-max product and computed the total degree and $v_{i}$-the total degree of a vertex in the lexicographic-max product of fuzzy graph structures. Furthermore, we have presented applications of fuzzy graph structures in decision-making, regarding detection of marine crimes and detection of the road crimes. We aim to broaden our work to (1) rough fuzzy graph structures, (2) rough fuzzy soft graph structures, and (3) fuzzy soft graph structures.

\section{Data Availability}

No data were used to support this study.

\section{Conflicts of Interest}

The authors declare that they have no conflicts of interest regarding the publication of this research article.

\section{Acknowledgments}

This research was financially supported by the Faculty of Science, Jazan University.

\section{References}

[1] L. A. Zadeh, "Fuzzy sets," Information and Control, vol. 8, no. 3, pp. 338-353, 1965.

[2] J. Clark and D. A. Holton, A First Look at Graph Theory, Allied Publishers Limited, Berlin, Germany, 1991.

[3] J. L. Gross and J. Yellen Dinesh, Graph Theory and its Applications, CRC Press, New York, NY, USA, 1998.

[4] A. Kauffman, "Introduction a la Theorie des Sous-emsembles Flous," Masson et Cie, vol. 1, 1973.

[5] A. Rosenfeld, Fuzzy Graphs, Fuzzy Sets and Their Applications, L. A. Zadeh, K. S. Fu, and M. Shimura, Eds., pp. 77-95, Academic Press, New York, NY, USA, 1975.

[6] L. A. Zadeh, "Similarity relations and fuzzy orderings," Information Sciences, vol. 3, no. 2, pp. 177-200, 1971.

[7] P. Bhattacharya, "Some remarks on fuzzy graphs," Pattern Recognition Letters, vol. 6, no. 5, pp. 297-302, 1987.

[8] M. S. Sunitha and A. Vijayakumar, "Complement of a fuzzy graph," Indian Journal of Pure and Applied Mathematics, vol. 33, no. 9, pp. 1451-1464, 2002.

[9] J. N. Mordeson and P. S. Nair, Fuzzy Graphs and Fuzzy Hypergraphs, Physica Verlag, Heidelberg, 1998.
[10] A. Nagoor Gani and K. Radha, "On regular fuzzy graphs," Journal of Physical Sciences, vol. 12, pp. 33-40, 2008.

[11] A. Nagoor Gani and K. Radha, "The degree of a vertex in some fuzzy graphs," International Journal of Algorithms, Computing and Mathematic, vol. 2, no. 3, pp. 107-116, 2009.

[12] K. R. Bhutani and A. Battou, "On M-strong fuzzy graphs," Information Sciences, vol. 155, no. 1-2, pp. 103-109, 2003.

[13] Q. J. Chen, "Matrix representation of fuzzy graphs," Information Sciences, vol. 1, pp. 41-46, 1990.

[14] M. Akram, m-Polar Fuzzy Graphs, Studies in Fuzziness and Soft Computing, Springer, Berlin, Germany, 2019.

[15] M. Akram, A. Habib, and J. C. R. Alcantud, "An optimization study based on dijkstra algorithm for a network with trapezoidal picture fuzzy numbers," Neural Computing and Applications, vol. 34, 2020.

[16] M. Akram and A. Luqman, "Granulation of ecological networks under fuzzy soft environment," Soft Computing, vol. 24, no. 16, pp. 11867-11892, 2020.

[17] B. M, S. Mathew, and J. N. Mordeson, "Wiener index of a fuzzy graph and application to illegal immigration networks," Fuzzy Sets and Systems, vol. 384, pp. 132-147, 2020.

[18] L. Kóczy, "Fuzzy graphs in the evaluation and optimization of networks," Fuzzy Sets and Systems, vol. 46, no. 3, pp. 307-319, 1992.

[19] S. Mathew and M. S. Sunitha, "Types of arcs in a fuzzy graph," Information Sciences, vol. 179, no. 11, pp. 1760-1768, 2009.

[20] S. Mathew, J. N. Mordeson, and D. S. Malik, "Fuzzy graph theory," Studies in Fuzziness and Soft Computing, vol. 363, 2018.

[21] J. N. Mordeson and P. Chang-Shyh, "Operations on fuzzy graphs,” Information Sciences, vol. 79, no. 3-4, pp. 159-170, 1994.

[22] J. N. Mordeson and P. S. Nair, "Cycles and cocycles of fuzzy graphs," Information Sciences, vol. 90, no. 1-4, pp. 39-49, 1996.

[23] M. L. N. McAllister, "Fuzzy intersection graphs," Computers \& Mathematics with Applications, vol. 15, no. 10, pp. 871-886, 1988.

[24] P. S. Nair, "Triangle and parallelogram laws on fuzzy graphs," Pattern Recognition Letters, vol. 15, no. 8, pp. 803-805, 1994.

[25] A. Nagoor Gani and K. Radha, "Conjunction of two fuzzy graphs," International Review of Fuzzy Mathematics, vol. 3, pp. 61-71, 2008.

[26] M. S. Sunitha and A. Vijayakumar, "A characterization of fuzzy trees," Information Sciences, vol. 113, no. 9, pp. 293-300, 1999.

[27] E. Takeda, "Connectivity in fuzzy graphs," Information Sciences, vol. 23, pp. 343-352, 1973.

[28] R. T. Yeh and S. Y. Bang, "Fuzzy relations, fuzzy graphs and their applications to clustering analysis," in Fuzzy Sets and Their Applications to Cognitive and Decision Process, L. A. Zadeh, K. S. Fu, K. Tanaka, and M. Shimura, Eds., pp. 125-149, Academic Press, New York, NY, USA, 1975.

[29] X. Yu and Z. Xu, "Graph-based multi-agent decision making," International Journal of Approximate Reasoning, vol. 53, no. 4, pp. 502-512, 2012.

[30] S. A. Edalatpanah, "Neutrosophic structured element," Expert Systems, vol. 1, 1990.

[31] S. A. Edalatpanah, "Neutrosophic perspective on DEA," Journal of Applied Research on Industrial Engineering, vol. 5, no. 4, pp. 339-345, 2018.

[32] S. A. Edalatpanah, "Systems of neutrosophic linear equations," Neutrosophic Sets and Systems, vol. 33, no. 1, p. 6, 2020. 
[33] S. Samanta and B. Sarkar, "A study on generalized fuzzy graphs," Journal of Intelligent \& Fuzzy Systems, vol. 35, no. 3, pp. 3405-3412, 2018.

[34] S. Samanta, B. Sarkar, D. Shin, and M. Pal, "Completeness and regularity of generalized fuzzy graphs," SpringerPlus, vol. 5, no. 1, pp. 1979-1990, 2016.

[35] E. Sampathkumar, "Generalized graph structures," Bulletin of Kerala Mathematics Association, vol. 3, no. 2, pp. 65-123, 2006.

[36] F. Hausdorff, Grundzge Mengenlehre, Veit and Company, Leipzig

[37] K. Radha and S. Arumugam, "On Lexicographic products of two fuzzy graphs," International Journal of Fuzzy Mathematical Archive, vol. 7, no. 2, pp. 169-176, 2015.

[38] T. Dinesh, A Study on Graph Structures, Incidence Algebras and Their Fuzzy Analogues, Kannur University, Kannur, India, 2011.

[39] R. V. Ramakrishnan and T. Dinesh, "On generalized fuzzy graph structures," Applied Mathematical Sciences, vol. 5, no. 4, pp. 173-180, 2011.

[40] R. V. Ramakrishnan and T. Dinesh, "On generalized fuzzy graph structures II," Advances in Fuzzy Mathematics, vol. 6, no. 1, pp. 5-12, 2011.

[41] R. V. Ramakrishnan and T. Dinesh, "On generalized fuzzy graph structures III," Bulletin of Kerala Mathematics Association, vol. 8, no. 1, pp. 57-66, 2011.

[42] M. Akram and M. Sitara, "Certain fuzzy graph structures," Journal of Applied Mathematics and Computing, vol. 61, 2019.

[43] M. Sitara, M. Akram, and M. Yousaf Bhatti, "Fuzzy graph structures with application," Mathematics, vol. 7, no. 1, p. 63, 2019.

[44] M. Akram, M. Sitara, and A. B. Saeid, "Residue product of fuzzy graph structures," Journal of Multiple-Valued Logic and Soft Computing, vol. 34, no. 3-4, pp. 365-399, 2020. 